\title{
Commented catalogue of Cassidinae (Coleoptera: Chrysomelidae) of the state of São Paulo, Brazil, with remarks on the collection of Jaro Mráz in the National Museum in Prague
}

\author{
Lukáš SEKERKA \\ Department of Entomology, National Museum, Cirkusová 1740, CZ-193 00, Praha - Horní Počernice, Czech Republic; \\ e-mail: sagrinae@gmail.com
}

Accepted:

$14^{\text {th }}$ December 2020

Published online:

$26^{\text {th }}$ December 2020

\begin{abstract}
Commented catalogue of Cassidinae species reported from the state of São Paulo, Brazil is given. Altogether, 343 species are presently registered from the state representing the following tribes: Alurnini (5 spp.), Cassidini (84 spp.), Chalepini (85 spp.), Dorynotini (9 spp.), Goniocheniini (8 spp.), Hemisphaerotini (2 spp.), Imatidiini (25 spp.), Ischyrosonychini (6 spp.), Mesomphaliini (83 spp.), Omocerini (14 spp.), Sceloenoplini (9 spp.), and Spilophorini (13 spp.). Fifty-two species are recorded for the first time and 19 are removed from the fauna of São Paulo. Each species is provided with a summary of published faunistic records for São Paulo and its general distribution. Dubious or insufficient records are critically commented. A list of Cassidinae species collected in São Paulo by Jaro Mráz (altogether 145 identified species) is included and supplemented with general information on this material. In addition, two new synonymies are established: Cephaloleia caeruleata Baly, $1875=$ C. dilatata Uhmann, 1948, syn. nov.; Stolas lineaticollis $($ Boheman, 1850) $=$ S. silaceipennis (Boheman, 1862), syn. nov.; and the publication year of the genus Heptatomispa Uhmann, 1940 is corrected to 1932. The following 54 species are recorded from São Paulo for the first time: Agroiconota tristriata (Fabricius, 1792), Charidotella (Philaspis) polita (Klug, 1829), Charidotis admirabilis Boheman, 1855, C. auroguttata Boheman, 1855, C. circumscripta Boheman, 1855, C. concentrica (Boheman, 1855), C. consentanea (Boheman, 1855), C. gemellata Boheman, 1855, Coptocycla (s. str.) stigma (Germar, 1823), Coptocycla (Coptocyclella) adamantina (Germar, 1823), Eremionycha bahiana (Boheman, 1855), Helocassis flavorugosa (Boheman, 1855), Helocassis flavorugosa (Boheman, 1855), Microctenochira patruelis (Boheman, 1855), Plagiometriona deyrollei (Boheman, 1855), P. punctatissima (Boheman, 1855), P. tenella (Klug, 1829), Baliosus conspersus Weise, 1911, Chalepus aenescens Weise, 1910, Decatelia pallipes (Weise, 1922), Octhispa gemmata (Germar, 1823), Octhispa robinsonii (Baly, 1864), Octotoma brasiliensis Weise, 1921, Octotoma crassicornis Weise, 1910, Oxychalepus centralis Uhmann, 1940, Temnochalepus imitans Uhmann, 1935, Uroplata coarctata Weise, 1921, Uroplata minuscula (Chapuis, 1877), Calliapis umbonata Hincks, 1956, Cephaloleia caeruleata Baly, 1875, C. flavovittata Baly, 1859, C. trilineata Uhmann, 1942, C. zikani Uhmann, 1935, Stenispa vespertina Baly, 1877, S. viridis (Pic, 1931), Xenispa bicolorata (Uhmann, 1948), Anacassis candida (Boheman, 1854), Chelymorpha commutabilis Boheman, 1854, C. constellata (Klug, 1829), Cyrtonota vulnerata (Boheman, 1850), Hilarocassis evanida (Boheman, 1850), Mesomphalia gibbosa (Fabricius, 1781), Nebraspis corticina (Boheman, 1850), Sceloenopla rectelineata (Pic, 1929), Stolas acuta (Boheman, 1850), S. aenea (Olivier, 1790), S. sexsignata (Boheman, 1850), S. sommeri (Boheman, 1850), S. subreticulata (Boheman, 1850), Omocerus (Platytauroma) cornutus (Boheman, 1850), Calyptocephala nigricornis (Germar, 1823), Oediopalpa brunnea (Uhmann, 1943), O. caerulescens (Baly, 1875), and O. fulvipes Baly, 1859.
\end{abstract}

Key words. Coleoptera, Chrysomelidae, Cassidinae, catalogue, new synonymy, Jaro Mráz, São Paulo, Brazil, Neotropical Region 


\section{Introduction}

Cassidinae with almost 6,400 described species are the second largest subfamily of Chrysomelidae. They have worldwide distribution with most of the diversity in tropical regions, which rapidly decreases towards the poles. In the past, the subfamily was separated into two distinct subfamilies, Cassidinae and Hispinae, but naturally they form just a single group with the true cassidines nested inside the hispines, and the name Cassidinae applies to both (e.g. SeKERKa 2010).

The diversity of Cassidinae is almost equally divided between the Old and the New World, the latter with 3,180 known species. Brazil is the most species rich country in the world with 1,478 species registered in Fauna do Brasil completed in 2015 (SEKERKa 2020). Since then, several new species were described while some others were synonymized and as a result, currently there are 1,471 species registered from Brazil (Sekerka, unpubl. data). The species count will increase in the future as there are many species, even some common ones, known from the neighbouring countries but not yet recorded from Brazil. Moreover, there are certainly hundreds of undescribed species waiting to be collected and discovered. On the other hand, many taxa described from Brazil are known based only on the type material that was not revised by a subsequent specialist, so the actual number of valid species is probably lower at the moment. This is basically already shown by the species number that dropped from the 2015 figures; even though eight new species have been described, twice as many have been synonymized (e.g. SEKERKA 2016).

Despite its enormous diversity, the cassidine fauna of Brazil is still poorly known and many species were registered for the first time as late as in 1990's. This is caused by the fact that previous workers on the cassidines did not have particular interest in publishing faunistic records. For example, two of the most prolific workers of their time, Julius Weise and Franz Spaeth, only rarely published faunistic records for individual Brazilian states unless they described the respective material as a new nominal taxon. This changed for the hispines in the 1930s as Erich Uhmann valued faunistic records and published them intensively, but for the true cassidines it had to wait until Lech Borowiec started to publish large faunistic papers making available the information he gathered during identification of material from various collections (BOROWIEC 1996, 2002, 2009; BOROWIEC \& TAKIZAWA 2011). However, with the increasing number of faunistic records, the number of erroneous ones also increased as some of the records were not critically evaluated with regard to the biogeography.

Within Brazil, catalogues were published for three states only: Amapá (Uhmann 1965, hispines), Rio de Janeiro (Flinte et al. 2009, cassidines s. str.), São Paulo (Uhmann 1964, hispines), and they never covered the entire subfamily in its present phylogenetic frame. Recently I processed material collected by Jaro Mráz in São Paulo deposited in the National Museum, Prague, and noticed it contained numerous species not reported from that state so far. Also, occurrence of many other species published in a wide array of local papers dealing with various aspects of biology of Cassidinae was not reflected in subsequent catalogues and thus their presence in São Paulo formally remained unknown. The aim of the present paper was to collect all published faunistic information on Cassidinae from São Paulo and produce a critical catalogue for the entire subfamily supplemented with new faunistic records. Currently, the Cassidinae fauna of São Paulo consists of 343 registered species, 54 of them being recorded for the first time. Occurrence of five species is doubtful, based on historic material only, and should be verified with new material. Finally, 19 species are removed from the fauna of the state.

\section{Material and methods}

The present catalogue is based on a list I compiled in 2015 for the Catálogo Taxonomico da Fauna do Brasil (SeKerka 2020). However, since then, there have been numerous taxonomic and distributional changes, which are included here. I have also verified numerous publications dealing mainly with biological aspects of various species that I did not have when I was compiling the list.

The classification follows BorowIEC (1999), BOROWEC \& Świętojańska (2020), Uhmann (1957), and Staines (2002) with recent modifications published by subsequent authors. Species are listed by tribes and organized alphabetically within each tribe. Each species in this catalogue is provided with an overview of faunistic records and distribution, when necessary also other paragraphs are added. List of published faunistic records is organized chronologically. Each locality is followed by a semicolon; a period is used when the locality had more information; a hyphen is used to connect a city and the respective locality. Finally, the locality(ies) is followed with a citation of the respective publication. Each locality was verified, whether it is truly situated in the state of São Paulo; where necessary minor variations of the original spelling were changed to the presently used one. However, when the spelling was considerably different, or I was unable to find it on any maps or gazetteers, it is given in single quotations in the original spelling; in the case of different/erroneous spelling my interpretation is placed within square brackets. The locality São Paulo is used for the entire state; if the authors mentioned that it stands for the city of São Paulo it is listed as São Paulo Capital. If the record was published under a different taxon name (i.e. synonym or misidentification), the original name is given in the parentheses with the respective reference. The synonyms are not listed unless they are further discussed; for full synonymy of the mentioned taxa see the abovementioned catalogues.

Distribution is based on published data only and follows the published catalogues mentioned earlier supplemented with additional records published elsewhere; for Brazilian species the general distribution is also given in SEKERKA (2020). Occurrence in particular Brazilian states is given in parentheses; if Brazil is mentioned alone the species has no detailed record. Occurrence in other countries is given only summarily, i.e. without province/department records. The occurrence in São Paulo as well as in other states/countries is critically reviewed and commented when necessary. 
Species excluded from the fauna of São Paulo are listed after the main catalogue and are organized alphabetically. A summary of all faunistic changes is presented after the main catalogue.

Type material is listed for taxa described from the material collected by Jaro Mráz and deposited in NMPC or if they were examined along with the herein proposed taxonomic changes. The label data are recorded verbatim as they appear on the labels. A single vertical bar '|' separates rows within a single label while a double vertical bar ' $\|$ ' separates individual labels. The labels are described using the following abbreviations placed in square brackets following each label, eventually row: bf - black frame, $\mathrm{cb}$ - cardboard, hw - handwritten, $\mathrm{p}$ - printed, $\mathrm{r}$ - red, $\mathrm{s}$ - soft, $\mathrm{t}$ - typed, $\mathrm{w}$ - white.

New records are provided for all species newly recorded for São Paulo while Additional records contain new unpublished material for species already reported from São Paulo. In both, the locality data are interpreted and given in the present-day spelling. Specimens vaguely labelled only as São Paulo, which thus may refer to the entire state are presented only when they represent a new state record.

Remarks contain additional information, mainly clarifications on distribution, new synonymies and any other comments.

Material collected by Jaro Mráz. The material was vaguely labelled only as 'São Paulo' (Figs 6-7) without any further data and thus its faunistic relevance is very limited. Therefore, each species collected by Mráz is only denoted with an asterisk without the complete record that is given only in case of a new faunistic record for São Paulo. Altogether, there are 145 species he found that I was able to identify. Further ten species ( 7 spp. of Chalepini, 2 spp. of Imatidiini and one species of Spilophorini) I have been unable to identify so far, mainly due to lack of more comparative material and unavailability of some type specimens. Voucher specimens of material collected by J. Mráz are primarily deposited in NMPC. However, duplicate specimens were retained by the specialists for identifications they provided, and also specimens of species present in longer series were exchanged with other institutions. As a result, Mráz's material can be found in nearly all European principal natural history museums (e.g. London, Paris, Berlin, Manchester, Bruxelles, Wien) as well as in U.S. (e.g. Chicago, Washington, etc.) and many other collections. See more information in the chapter about Brazilian collections of J. Mráz.

List of abbreviations for collections mentioned in the catalogue:

BMNH The Natural History Museum, London, United Kingdom (M. Barclay, M. Geiser);

KVSC Collection of Károly Vig, Szombathely, Hungary;

LSPC Collection of Lukáš Sekerka, Prague, Czech Republic;

MNHN Muséum National d'Histoire Naturelle, Paris, France (A. Mantilleri);

MNRJ Museu Nacional, Universidade Federal, Rio de Janeiro, Brazil (M. and M. Monné);

MZUSP Museu de Zoologia da Universidade de São Paulo (G. Biffi); NHMB Naturhistorisches Museum, Basel, Switzerland (M. Borer);

NMPC National Museum, Prague, Czech Republic;

NHMW Naturhistorisches Museum, Wien, Austria (H. Schilhammer);
NHRS Naturhistoriska Riksmuseet, Stockholm, Sweden(J. Bergsten);

OKZC Collection of Ondřej Konvička, Zlín, Czech Republic;

SDEI Senckenberg Deutsches Entomologisches Institut, Müncheberg, Germany (K. Nadein);

SMNS Staatliche Museum für Naturkunde, Stuttgart, Germany (W. Schawaller);

USNM National Museum, Smithsonian Institution, Washington D.C., USA (A, Konstantinov);

ZSMC Zoologische Staatsammlung, München, Germany (M. Balke).

\section{Results}

\section{Brazilian collection of Jaro Mráz with emphasis on Cassidinae material}

Jaro Mráz (1880-1927) was a Czech lithographer working in São Paulo, Brazil. He had an eventful life full of challenges, not only due to his enthusiasm and passion for nature. Before he came to Brazil, he worked in Scandinavia, Germany, Belgium, and Spain. In Brazil, where he first worked in a large company before he was able to run his own business, he was so fascinated by nature that he immediately started to collect natural history objects upon his arrival (OBENBERGER 1927a).

Jaro Mráz lived in Santa Anna, at that time a small town situated north of São Paulo downtown across the Tietê River. It was a rather rural and isolated settlement mainly due to natural barriers, the Tietê River to the south and Serra da Cantareira to the north. But since the beginning of the $20^{\text {th }}$ century, it has developed rapidly and turned completely to an urban zone. Nowadays Santana is an important commercial and residential district and one of the fastest growing ones in São Paulo.

Shortly after the World War I, Jan Obenberger, one of the most enthusiastic and prolific Czech entomologists and a leading expert in taxonomy of jewel beetles, started to think how to enrich entomological collections of the newly created Department of Entomology of the National Museum in Prague. He published an appeal in newspapers motivating Czechoslovak citizens living or travelling to foreign countries to collect insects for the National Museum. This endeavour failed in most cases, but it resulted in a few productive cooperations. According to OBENBERGER (1927a,b), this endeavour was mocked by a certain person, who visited the Czechoslovak community in São Paulo, and upon this Mráz wrote a letter to Obenberger offering that he would collect for him free of any charges and so their collaboration started back in 1920/1921. They corresponded frequently and Mráz almost immediately started to send material he collected. By 1922 it was already 20-30 thousand specimens, mainly small beetles, which Mráz collected by sifting leaf litter. At the same time, Obenberger informed Mráz that his material would officially become part of the museum collections and Mráz promised he would collect exclusively for the National Museum and he would send also his earlier collections (OBENBERGER 1922). In subsequent years they exchanged correspondence frequently and Obenberger informed Mráz about various collecting techniques and turned his attention particularly to buprestids, which were objects of his interest. Concurrently, Mráz was very interested in course of events 
in the young Czechoslovakia and their correspondence became more familiar. However, he had only a little time to collect, usually only on Sundays, and otherwise he had to work hard to support his family. Yet he refused any compensation for his collecting efforts and even sent the packages on his own expenses. Obenberger described him as a kind and honourable but proud person refusing any financial compensation for his collecting activities, even though not infrequently he had a little money for himself. Their collaboration lasted until the death of Mráz on 22 July 1927 . However, his legacy will live forever as he sent to the National Museum about 200 shipments with insects only estimated to 300,000 specimens, which at that time represented perhaps the largest collection from Brazil (OBenberger 1927a,b).

As the time passed by, Mráz became a keen observer and an experienced collector sending a wide array of mainly smaller beetles, and even many foreign researchers were surprised by the richness and diversity of the material collected by him. But he collected also other insects, reptiles, spiders, lichens etc. Frequently, in letters he provided also detailed information on the material he sent and photographs of localities, where he collected. Unfortunately, this information was not kept with the specimens, which were all given a locality label stating only 'São Paulo, Mráz lgt.' (Figs 6-7).

It is disputable where exactly Mráz collected but assuming he had time to collect only during Sundays he must have collected mainly in Santana and its surroundings. On the other hand, his material is very diverse and thus he must have collected in many various habitats including mountains.

I have studied all specimens of Cassidinae (ca 2,300 specimens representing 145 identified species) collected by Mráz and deposited at the National Museum. Based on these and other records known to me, and on species requirements and their distributional patterns, I am quite sure that he must have collected in Serra da Cantareira, as many species he collected were reported by other authors from these mountains, including quite restrictive and characteristic species such as Mesomphalia sublaevis Boheman, 1850 or Coptocycla (Podostraba) ruficornis Spaeth, 1936; also, some Plagiometriona Spaeth, 1899 are known only from the internal mountain chains. Even at this time, the area around Santana was rural and rather dry, and that's probably where he got many species associated typically with savannas or cerrado, mainly grass feeding hispines which are usually not that abundant in forests. On the other hand, he collected also some montane species so far known only from the chain of Serra do Mar (e.g. Chlamydocassis subcornuta (Boheman, 1850), Cyrtonota vulnerata (Boheman, 1850), some Plagiometriona etc.). Nevertheless, these species are rather rare in collections and their distribution is far from being understood. Very interesting is large material of Cephaloleia vittipennis Weise, 1910 (over 200 specimens), which is known to me from lowlands along the seacoast and eastern slopes of Serra do Mar. These specimens morphologically exactly match the type series collected in Santos, so it would be interesting to know whether there are similar cases also in other insect or other animal groups Mráz collected, which would mean that he might have visited lowlands east of Serra do Mar. I was trying to locate diaries and letters Mráz sent to Obenberger to possibly enlighten where exactly he might have collected but their whereabouts are presently unknown. On the other hand, the documentary bequest of Obenberger is vast and not properly organized, thus there is still a chance that some documents might be recovered in the future.

The Cassidinae material collected by Mráz was partially processed by Franz Spaeth, who described several new species based on this material (Charidotella (s.str.) recidiva (Spaeth, 1926), Charidotis aerosa Spaeth, 1936, Charidotis mrazi Spaeth, 1936, Coptocycla (Podostraba) ruficornis Spaeth, 1936, Microctenochira triplagiata (Spaeth, 1926), Stolas perfuga (Spaeth, 1926), and Stolas selecta (Spaeth, 1928)). However, Spaeth probably never saw the material complete as it was sent gradually during the period 1921-1927. He published only descriptions of the new species he recognized but he often did so a decade after he had identified and returned the material. For his service he received duplicate specimens for his collection, which he often further exchanged, so nowadays, the material is deposited in various museums. He also identified part of the hispines but he was not capable to process all of them and send duplicates he retained to Erich Uhmann, who described another two species ( $\mathrm{Ce}$ phaloleia impressa Uhmann, 1930 and C. nitida Uhmann, 1930) but unfortunately without inclusion of the NMPC material. Spaeth never produced a complex publication devoted to this material as he did not value new faunistic records, and as a consequence, many species are here reported from São Paulo for the first time.

\section{Catalogue of species of Cassidinae reported from São Paulo}

(species denoted with an asterisk were collected by Jaro Mráz)

\section{Tribe Alurnini}

\section{*Coraliomela aeneoplagiata (Lucas, 1859)}

Published records. São Paulo (WeISE 1900, as Silurnus consanguinea Weise 1900); Amparo; Campinas; Cotia; Jundiaí; Rio Claro; São Carlos; São Paulo Capital (Fischer 1935); Campinas (Mendes 1938); Rio Claro (Buck 1958); Piracicaba (Toledo Piza 1968).

Distribution. Brazil (Goiás, Minas Gerais, Rio de Janeiro, São Paulo).

\section{Coraliomela brunnea (Thunberg, 1821)}

Published records. Piracicaba (Bondar 1922); Amparo; Angatuba; Anhangaí; Avanhandava; Ipiranga; Piracicaba (FISCHER 1935); ItuFazenda Pau d'Alho (Uhmann 1964); Piracicaba (Toledo Piza 1968); Itu; Mogi Mirim-Fazenda Campininha; Piracicaba; São Paulo-Ipiranga (JUNG \& MONNÉ 2006).

Distribution. Argentina, Bolivia, Brazil (Amazonas, Bahia, Ceará, Goiás, Maranhão, Minas Gerais, Mato Grosso, Mato Grosso do Sul, Pará, Paraná, Paraíba, Pernambuco, Piauí, Rio Grande do Norte, Rio Grande do Sul, Rio de Janeiro, Rondônia, Santa Catarina, São Paulo, Sergipe), Paraguay, and Uruguay. 


\section{Coraliomela quadrimaculata \\ (Guérin-Méneville, 1840)}

Published records. Santos; São Paulo (Bondar 1940).

Distribution. Argentina, Bolivia, Brazil (Rio de Janeiro, Rio Grande do Sul, Santa Catarina, São Paulo), and Paraguay.

\section{Mecistomela marginata (Thunberg, 1821)}

Published records. São Paulo (Bondar 1913); Santos (BondAr 1922); Bocaina (Uhmann 1932); Alto da Serra; Jundiaí; São Paulo Capital; São Paulo-Santo Amaro (FisCHER 1935).

Distribution. Argentina, Brazil (Bahia, Distrito Federal, Espírito Santo, Goiás, Minas Gerais, Paraná, Rio Grande do Sul, Rio de Janeiro, Santa Catarina, São Paulo), and Paraguay.

\section{Platyauchenia latreillei (Laporte de Castelnau, 1840)}

Published record. São Paulo (BondAR 1940, STAINES 2007).

Distribution. Brazil (Bahia, Rio de Janeiro, São Paulo).

Tribe Cassidini

Agroiconota gibberosa (Boheman, 1855)

Published records. Salto Grande (Boheman 1855); Parque do Estado (Borowiec 2005).

Distribution. Brazil (Minas Gerais and São Paulo).

$$
\text { *Agroiconota inedita (Boheman, 1855) }
$$

Published records. Bertioga; Santa Anna; São Paulo Capital (BorowIEC 2005); Piracicaba; Saltinho (Lopes et al. 2016).

Distribution. Argentina, Bolivia, Brazil (Mato Grosso, Minas Gerais, Pará, Paraná, Pernambuco, Rio de Janeiro, Santa Catarina, São Paulo), and Paraguay.

\section{Agroiconota lateripunctata Spaeth, 1936}

Published record. 'Mendes [= name of collector; thus, São Paulo only]' (BOROWIEC 1996).

Distribution. Argentina, Bolivia, Brazil (Bahia, Mato Grosso do Sul, São Paulo), Paraguay, and Venezuela.

Remarks. BorowIEC (1996) published material labelled 'Sao Paulo Mendes' and interpreted Mendes as the name of locality. However, it is most likely a name of collector rather than locality (L. Borowiec, pers. comm. 2020) and thus, the record is here interpreted as São Paulo only.

\section{*Agroiconota pullula (Boheman, 1855)}

Published records. Boicaina (BorowIEC 1996); 'Borto flor.'; Cantareira; Jabaquara; Santo Amaro (BorowIEC 2005).

Distribution. Bolivia, Brazil (Minas Gerais, Rio de Janeiro, São Paulo), Paraguay, and Peru.

\section{Agroiconota urbanae Buzzi, 1996}

Published record. Jundaí-Serra do Japi (Buzzi 1996).

Distribution. Brazil (Mato Grosso do Sul and São Paulo). Remarks. This species was considered a synonym of $A$. sub- vittata (Boheman, 1855) due to misidentification of the latter species. SEKERKa (2016) clarified the situation, restored its species status and updated its distribution.

\section{*Agroiconota tristriata (Fabricius, 1792)}

New record. BRAZIL: São Paulo: without further data, 1 spec., J. Mráz leg. (NMPC).

Distribution. Argentina, Brazil (Amazonas, Maranhão, Mato Grosso, Minas Gerais, Pará, Santa Catarina), French Guiana, Peru, Paraguay, Suriname, Trinidad and Tobago, and Venezuela. New to São Paulo.

\section{Aporocassida graphica (Germar, 1823)}

Published record. São Paulo (BoHEMAN 1854).

Distribution. Argentina, Brazil (São Paulo), and Uruguay.

Remarks. Aporocassida graphica is one of the most common species of Cassidinae in Argentina, where it has been recorded from NW provinces south to Buenos Aires and from Uruguay (ŚwIĘTOJAŃSKA 2002). The record from São Paulo was published by BoHEMan (1854) but was not repeated by subsequent authors (i.e., SPAETH 1914, Borowiec 1999, ŚwIĘTOJAŃSKa 2002), although Świętojańska listed several specimens vaguely localized as from Brazil. The presence of $A$. graphica in Brazil (as well as in Paraguay) is quite predictable; so far, the closest record is from Resistencia in the province of Chaco (BoRowIEC 1996), which is basically at the border with Paraguay and circa $300 \mathrm{~km}$ (air distance) from the Brazilian borders of Rio Grande do Sul. On the other hand, the occurrence of A. graphica in São Paulo and thus in Brazil should be considered dubious until it is confirmed by new material.

*Charidotella (s. str.) conclusa (Boheman, 1855)

Published record. Amparo (BOROwIEC 1996).

Distribution. Brazil (São Paulo).

\section{Charidotella (s. str.) hoegbergi (Boheman, 1855)}

Published records. Barueri; São Paulo (BorowIEC 1996).

Distribution. Argentina, Brazil (Mato Grosso, Mato Grosso do Sul, Pará, Paraná, Rio de Janeiro, Rio Grande do Sul, São Paulo), Costa Rica, French Guiana, Mexico, Panama, and Paraguay.

\section{*Charidotella (s. str.) immaculata (Olivier, 1791)}

Published records. Cantareira (Borowiec 1996; as C. sejuncta (Boheman, 1855)); Mogi Guaçu-Fazenda Campininha; Ourinhos (BorowIEC 2002); Piracicaba; Rio Claro; Saltinho (Lopes et al. 2016).

Distribution. Argentina, Brazil (Goiás, Mato Grosso, Minas Gerais, Pará, Paraná, Pernambuco, Rio de Janeiro, Rio Grande do Sul, Roraima, Santa Catarina, São Paulo), Colombia, Dominican Republic, Ecuador, French Guiana, Paraguay, Peru, Suriname, Trinidad and Tobago, and Venezuela. 
Charidotella (s. str.) kesseli Borowiec, 1989

Published record. Bocaina (BorowIEC 1989).

Distribution. Brazil (São Paulo).

Charidotella (s. str.) limpida (Boheman, 1855)

Published record. Serra do Cantareira (BorowIEC 1996).

Distribution. Argentina, Bolivia, Brazil (Rio de Janeiro, São Paulo), Paraguay, and Peru.

*Charidotella (s. str.) morio (Fabricius, 1801)

Published record. São Paulo (Borowiec 1996).

Distribution. Brazil (Minas Gerais, Rio de Janeiro, São Paulo).

*Charidotella (s. str.) recidiva (Spaeth, 1926)

(Fig. 1)

Published records. São Paulo (SPAETh 1926a); Barueri (BorowIEC 1996); Jundiaí (BorowIEC 2002).

Type material examined. SyNTYPES: 1 spec. (Fig. 1), glued, 'SAÕ PAULO | BRAS. MRÁZ LGT. | MUS.PRAGENSE [w, p, cb, bf] || Metriona $\mid$ recidiva $\mid$ m. cotyp. $\mid$ n. sp. [w, hw by F. Spaeth, cb]' (NMPC); 2 spec., 'SAÕ PAULO | BRAS. MRÁZ LGT. | MUS.PRAGENSE [w, p, cb, bf] || Metriona | recidiva | Sp. | COTYPE [w, hw by J. Achard, cb]' (NMPC); 82 spec., 'SAÕ PAULO | BRAS. MRÁZ LGT. | MUS. PRAGENSE [w, p, cb, bf]' (NMPC). Each specimen was provided with an additional label: 'SYNTYPUS | Metriona | recidiva | Spaeth, 1926 | L. Sekerka des. 2008 [r, p, cb, bf]'.

Additional record. BRAZIL: São PAulo: Alto da Serra, 7.iii.1912, 1 spec., G. E. Bryant leg. (BMNH).

Distribution. Argentina and Brazil (Minas Gerais, Rio de Janeiro, Rio Grande do Sul, Santa Catarina, São Paulo).

Remarks. SPAETH (1926a) described C. recidiva based on extensive material from 'Sao Paulo (Mráz), St. Catharina (Böttcher), Rio Grande do Sul, Rio Janeiro' but did not mention precise number of specimens and their depositories. NMPC holds long series of specimens collected by J. Mráz and three specimens from Mendes in Rio de Janeiro (ex coll. J. Achard), but only one specimen from Mráz's series and two from Achard's collection have Spaeth's original identification labels which have 'cotype' written on them. Based on the primary description of $C$. recidiva, Spaeth did not select one representative as the 'type', therefore all specimens must be considered syntypes. As far as it is known to me, the series is consistent and there is no need for a lectotype designation now.

\section{*Charidotella (s. str.) sexpunctata (Fabricius, 1781)}

Published records. Barueri; Bosque da Saúde (Borowiec 1996); Saltinho (Lopes et al. 2016).

Additional record. BRAZIL: SÃo Paulo: Taubaté, 27.x.2005, 1 spec., Z. Tüdös leg. (OKZC).

Distribution. Argentina, Belize, Bolivia, Brazil (Amazonas, Bahia, Goiás, Maranhão, Mato Grosso, Mato Grosso do Sul, Minas Gerais, Pará, Paraná, Pernambuco, Rio Grande do Sul, Rio de Janeiro, Rondônia, Santa Catarina, São Paulo), Colombia, Costa Rica, Ecuador, El Salvador, French Guiana, Guatemala, Guiana, Honduras, Mexico,
Nicaragua, Panama, Paraguay, Peru, Suriname, Trinidad and Tobago, USA, and Venezuela.

Remarks. Charidotella sexpunctata as presently understood is a widely distributed species with many local forms. All specimens collected by J. Mráz belong to the form characterized by larger size of body, bright red/ orange-coloured elytra with large black spots in dead specimens.

\section{Charidotella (s. str.) virgo (Boheman, 1855)}

Published records. Salto Grande (Boheman 1855); Bosque da Saúde; São Paulo (Borowiec 1996).

Distribution. Argentina, Brazil (Paraná, São Paulo), and Paraguay.

\section{*Charidotella (Metrionaspis) rubicunda (Guérin-Méneville, 1844)}

Published records. Cantareira; 'Mendes [= collector; thus, São Paulo only]' (Borowiec 1996).

Distribution. Argentina, Bolivia, Brazil (Amazonas, Bahia, Mato Grosso, Mato Grosso do Sul, Minas Gerais, Paraná, Rio de Janeiro, São Paulo), Ecuador, Paraguay, and Peru.

Remarks. BorowiEC (1996) published material labelled 'Sao Paulo Mendes' and interpreted Mendes as the name of locality. However, it is most likely a name of collector rather than locality (L. Borowiec, pers. comm. 2020) and thus, the record is here interpreted as São Paulo only.

\section{Charidotella (Philaspis) polita (Klug, 1829)}

New record. BRAZIL: São Paulo: São Paulo, 1 spec. (BMNH).

Distribution. Brazil (Rio Grande do Sul). New to São Paulo.

Remarks. According to KLUG (1829: 2), the type material was collected (translated): 'in South Brazil, in the vicinity of Montevideo, Cassapava [= Caçapava do Sul], Porto Allegre by [Friedrich] Sellow'; the locality data were interpreted by following authors only as South Brazil. In the same work, Klug described another 18 species of Cassidinae, most of which are very common and widespread through the entire basin of the Paraná River. Their type specimens are morphologically similar to populations from Rio Grande do Sul while populations from the estuary of the Paraná River (including Montevideo area) are morphologically more or less distinct. Therefore, I assume that the Cassidinae were most likely collected in Porto Alegre or Caçapava do Sul, both located in the Brazilian state of Rio Grande do Sul.

\section{*Charidotella (Xenocassis) incerta (Boheman, 1855)}

Published records. Engenheiro Coelho; São Paulo (Simões \& Monné 2008).

Additional record. BRAZIL: São PAulo: Alto da Serra, 7.iii.1912, 1 spec., G. E. Bryant leg. (BMNH).

Distribution. Brazil (Minas Gerais, Rio de Janeiro, São Paulo). 


\section{Charidotis admirabilis Boheman, 1855}

New record. BRAZIL: São Paulo: São Paulo, 1 spec. (BMNH).

Distribution. Brazil (Santa Catarina). New to São Paulo.

*Charidotis aerosa Spaeth, 1936

(Fig. 3)

Published record. São Paulo (SPAEth 1936a).

Type material examined. SyntyPES: 1 spec. (Fig. 3), glued, 'SAÕ PAULO | BRAS. MRÁZ LGT. | MUS.PRAGENSE [w, p, cb, bf] || COTYPE [r, cb, hw] || Charidotis | aerosa m. cotyp. [hw by F. Spaeth] | Spaeth det. [w, cb, p]' (NMPC); 3 spec., glued, 'SAÕ PAULO | BRAS. MRÁZ LGT. | MUS.PRAGENSE [w, p, cb, bf] || COTYPE [r, hw, cb]' (NMPC). Each specimen was provided with an additional label: 'SYNTYPUS Charidotis | aerosa | Spaeth, 1936 | L. Sekerka des. 2008 [r, p, cb, bf]'.

Distribution. Brazil (São Paulo).

Remarks. SPAETH (1936a) described this species based on an unknown number of specimens from São Paulo and stated: 'coll. Spaeth, Typi; Mus. Prag, Mraz; Mus. Brit., Bryant; Mus. Ithaca, Hammar Coll.'. Because in the original description Spaeth did not select one specimen as the type, all specimens must be considered syntypes.

\section{*Charidotis annularis (Boheman, 1855)}

Published record. São Paulo (SPAETh 1936a, as C. signoreti Boheman, 1855).

Distribution. Brazil (Bahia, Minas Gerais, Paraná, Rio de Janeiro, São Paulo).

\section{Charidotis aurofasciata (Erichson, 1847)}

Published record. Teodoro Sampaio (BorowIEC 1996).

Distribution. Bolivia, Brazil (Amazonas, Pará, São Paulo), Colombia, Guatemala, French Guiana, Guyana, Honduras, Mexico, Nicaragua, Panama, and Peru.

\section{*Charidotis auroguttata Boheman, 1855}

New record. BRAZIL: São Paulo: without further data, 3 spec., J. Mráz leg. (NMPC).

Distribution. Argentina, Belize, Bolivia, Brazil (Amazonas, Goiás, Mato Grosso, Mato Grosso do Sul, Pará, Paraná, Rio Grande do Sul, Santa Catarina), Ecuador, Guatemala, Mexico, Panama, Paraguay, and Venezuela. New to São Paulo.

\section{Charidotis brevicollis Spaeth, 1936}

Published record. 'Mendes [= collector; thus, São Paulo only]' (BoROWIEC 1996).

Distribution. Brazil (Amazonas, ? São Paulo).

Remarks. BorowiEC (1996) published material labelled 'Sao Paulo Mendes' and interpreted Mendes as the name of locality. However, it is most likely a name of collector rather than locality (L. Borowiec, pers. comm. 2020) and thus, the record is here interpreted as São Paulo only.

Charidotis brevicollis was described from the locality 'S. Gabriel am Rio Negro', which refers to the municipality of São Gabriel da Cachoeira in the state of Amazonas. Occurrence of this species in São Paulo should be verified by additional material because most Charidotis Boheman, 1854 are forest species, usually quite restrictive concerning their habitat. Of all Brazilian species of Charidotis only the ones widespread and common in the entire tropical America (i.e., C. auroguttata, $C$. aurofasciata and $C$. vitreata (Perty, 1832)) are distributed in the Amazon and reaching also to SE Brazil.

\section{Charidotis candens (Boheman, 1855)}

Published record. São Paulo (SPAETh 1936a, as C. sanguinolenta (Swederus, 1787)).

Distribution. Brazil (Rio de Janeiro, São Paulo).

*Charidotis circumducta (Boheman, 1855)

Published records. Barueri; Cantareira (BOROwIEC 1996).

Distribution. Brazil (Rio de Janeiro, Rio Grande do Sul, Santa Catarina, São Paulo).

\section{*Charidotis circumscripta Boheman, 1855}

New record. BRAZIL: São PAULO: without further data, 1 spec., J. Mráz leg. (NMPC).

Distribution. Brazil (Mato Grosso, Paraná, Rio de Janeiro). New to São Paulo.

\section{Charidotis clypeolata Boheman, 1855}

Published record. São Paulo (BorowIEC 1996).

Distribution. Brazil (São Paulo) and Paraguay.

\section{*Charidotis concentrica (Boheman, 1855)}

New records. BRAZIL: São PAULo: without further data, 3 spec., J. Mráz leg. (NMPC); Alto da Serra, 9.iii.1912, 2 spec., G. E. Bryant leg. (BMNH)

Distribution. Brazil (Rio de Janeiro). New to São Paulo.

\section{Charidotis connexa (Boheman, 1855)}

Published record. São José do Rio Preto (Borowiec 2002)

Distribution. Brazil (Bahia, Paraná, São Paulo) and Bolivia.

*Charidotis consentanea (Boheman, 1855)

New records. BRAZIL: São PAULO: without further data, 9 spec., J. Mráz leg. (NMPC), 1 spec. (BMNH).

Distribution. Argentina, Brazil (Paraná, Rio Grande do Sul, Santa Catarina), and Paraguay. New to São Paulo.

\section{Charidotis crenata (Boheman, 1855)}

Published records. São Paulo (SPAETh 1936a); Jabaquara (BoROwIEC 1996).

Distribution. Brazil (São Paulo).

\section{*Charidotis furunculus (Boheman, 1855)}

Published records. Cantareira (Borowiec 1996); Mogi das Cruzes-Fazenda Carmelita (Borowiec 2002).

Distribution. Argentina and Brazil (Paraná, Rio de Janeiro, Rio Grande do Sul, Santa Catarina, São Paulo). 


\section{*Charidotis gemellata Boheman, 1855}

New records. BRAZIL: São Paulo: without further data, 4 spec., J. Mráz leg. (NMPC), 1 spec. (BMNH).

Distribution. Brazil (Paraná, Rio Grande do Sul, Santa Catarina). New to São Paulo.

\section{Charidotis laetabunda Spaeth, 1936}

Published record. São Paulo (SPAETh 1936a).

Distribution. Brazil (Espírito Santo, Santa Catarina, São Paulo).

\section{Charidotis laevisculpta Spaeth, 1936}

Published record. São Paulo (SPAETH 1936a).

Distribution. Brazil (São Paulo).

\section{*Charidotis mansueta (Boheman, 1855)}

Published records. Barueri (Borowiec 1996); Mogi Guaçu-Fazenda Campininha; Ourinhos (BorowIEC 2002).

Additional record. BRAZIL: São PAUlo: Campinas, v.1883, 1 spec., A. Fry leg. (BMNH)

Distribution. Argentina, Brazil (Minas Gerais, Paraná, Rio Grande do Sul, Santa Catarina, São Paulo), and Paraguay.

\section{*Charidotis marginella (Fabricius, 1775)}

Published records. Salto Grande (Boheman 1855, as C. herbida Boheman, 1855); 'Lurs de Golo'; São Paulo (Borowiec 1996, as C. punctatostriata Boheman, 1855).

Distribution. Argentina, Bolivia, Brazil (Mato Grosso, Mato Grosso do Sul, Paraná, Rio Grande do Sul, Santa Catarina, São Paulo), and Paraguay.

Remarks. Charidotis marginella was not formally reported from São Paulo; however, there are published records under two different nominal taxa which were recently synonymized with $C$. marginella; for more details see SEKERKA \& BARCLAY (2014)

\section{*Charidotis mrazi Spaeth, 1936}

$$
\text { (Fig. 2) }
$$

Published record. São Paulo (SPAETh 1936a).

Type material examined. SyNTYPE (Fig. 2): glued, 'SAÕ PAULO BRAS. MRÁZ LGT. | MUS.PRAGENSE [w, p, cb, bf] || TYPUS [r, p, cb, bf] || Charidotis | Mrazi m. Typus [hw by F. Spaeth] | Spaeth det. [w, cb, p] || SYNTYPUS | Charidotis | mrazi | Spaeth, 1936 | L. Sekerka des. $2020[\mathrm{r}, \mathrm{cb}, \mathrm{p}$ bf]' (NMPC)

Distribution. Brazil (Espírito Santo, Santa Catarina, São Paulo).

Remarks. SPAETH (1936a: 89) based the description on at least two specimens as he mentioned two localities and depositories: 'Sao Paulo (Mraz, Mus. Prague), Espirito Santo (coll. Spaeth)'. However, he did not explicitly fix the type, thus both (all) specimens are syntypes.

\section{Charidotis neglecta (Boheman, 1855)}

Published record. Ubatuba (SIMõES \& Monné 2008).

Distribution. Brazil (Rio de Janeiro, São Paulo).
*Charidotis nigrocincta Boheman, 1855

Published record. São Paulo (BOROWIEC 1996).

Distribution. Brazil (Rio de Janeiro, São Paulo).

Charidotis pupillata (Boheman, 1855)

Published record. Peruíbe (SIMões \& MONNÉ 2008).

Distribution. Brazil (Paraná, Santa Catarina, São Paulo).

\section{Charidotis rotundata Boheman, 1855}

Published record. São Paulo (SPAETH 1936a).

Distribution. Brazil (Rio de Janeiro, São Paulo).

Coptocycla (s. str.) auricoma Boheman, 1855

Published record. Cantareira (BORowIEC 1996).

Distribution. Brazil (Rio de Janeiro, São Paulo).

Coptocycla (s. str.) roseocincta Boheman, 1855

Published record. São Paulo (BorowIEC 1996).

Distribution. Brazil (Bahia, Pernambuco, São Paulo).

*Coptocycla (s. str.) stigma (Germar, 1823)

New records. BRAZIL: São Paulo: without further data, 1 spec., J. Mráz leg. (NMPC); Ilha de Santo Amaro-near Santos, 23.iii.1912, 2 spec., G. E. Bryant leg. (BMNH).

Distribution. Argentina, Bolivia, and Brazil (Mato Grosso, Pará, Paraná, Rio de Janeiro, Santa Catarina). New to São Paulo.

Remarks. All three specimens were identified as ' $\mathrm{Cop}$ tocycla undecimpunctata ab[erration]?' by F. Spaeth, probably because they have much larger spots on elytra than typical forms. However, the size, shape of the head and position of the spots perfectly agree with C. stigma.

\section{Coptocycla (s. str.) virguncula Boheman, 1862}

Published record. São Paulo (BoRowiec 2002).

Distribution. Brazil (Mato Grosso, São Paulo).

\section{*Coptocycla (Coptocyclella) adamantina} (Germar, 1823)

New records. BRAZIL: São PAulo: without further data, 4 spec., J Mráz leg. (NMPC); Capão Bonito, 13.xii.1978, 1 spec., J. H. Pedrosa leg. (SMNS); Ilha de Santo Amaro-near Santos, 17.iii.1912, 2 spec., G. E. Bryant leg. (BMNH); Santos, 1.iii.1912, 1 spec., G. E. Bryant leg. $(\mathrm{BMNH})$

Distribution. Argentina, Bolivia, Brazil (Distrito Federal, Mato Grosso do Sul, Minas Gerais, Pará, Paraná, Rio de Janeiro, Rio Grande do Sul, Santa Catarina), Paraguay, Peru, and Venezuela. New to São Paulo.

\section{Coptocycla (Podostraba) arcuata (Swederus, 1787)}

Published records. São Paulo (BoHEMAn 1857); Bocaina; Cantareira (BorowIEC 1996). 

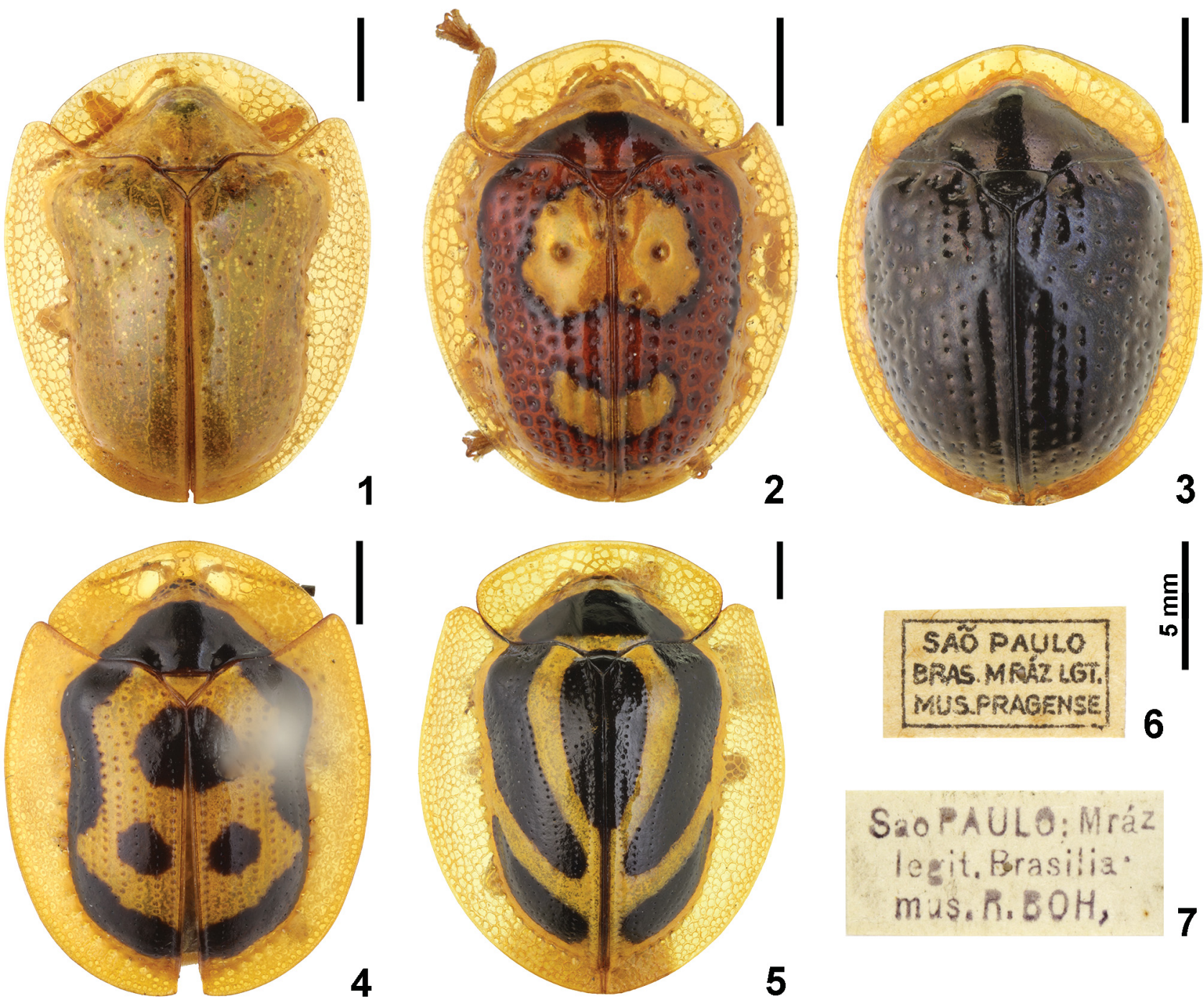

3

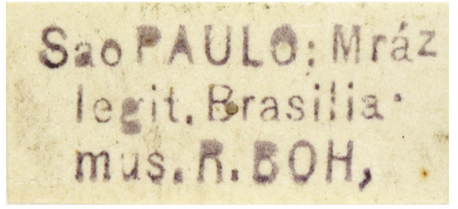

Figs 1-7. Dorsal aspects and labels. 1 - Charidotella (s.str.) recidiva (Spaeth, 1926), syntype; 2 - Charidotis mrazi Spaeth, 1936, syntype; 3 - Charidotis aerosa Spaeth, 1936, syntype; 4 - Microctenochira triplagiata (Spaeth, 1926), holotype; 5 - Coptocycla (Podostraba) ruficornis Spaeth, 1936, syntype; 6-7 - typical locality labels for Mráz’s material. Scale bars unless stated $=1 \mathrm{~mm}$.

Distribution. Brazil (Minas Gerais, Rio de Janeiro, Santa Catarina, São Paulo).

Remarks. The material published by Boheman needs to be verified as since that time SPAETH (1936b) described another three species belonging to this group.

\section{*Coptocycla (Podostraba) ruficornis Spaeth, 1936} (Fig. 5)

Published records. São Paulo (SPAETH 1936b); Cantareira (Borowiec 2002). Type material examined. SYNTYPES: 2 spec., glued, 'SAÕ PAULO | BRAS. MRÁZ LGT. | MUS.PRAGENSE [w, p, cb, bf] || Coptocycla | arcuata Sw. [hw by F. Spaeth] | Spaeth det. [w, p, cb]' (NMPC); 1 spec., glued, 'SAÕ PAULO | BRAS. MRÁZ LGT. | MUS.PRAGENSE [w, p, cb, bf] || Coptocycla | arcuata [hw by F. Spaeth] | Spaeth det. [w, cb, p]' (NMPC); 14 spec., glued, 'SAÕ PAULO | BRAS. MRÁZ LGT. | MUS. PRAGENSE [w, p, cb, bf]' (NMPC). Each specimen was provided with an additional label: 'SYNTYPUS | Coptocycla | ruficornis | Spaeth, 1936 | L. Sekerka des. 2020 [r, p, cb, bf]'.

Distribution. Brazil (São Paulo).

Remarks. SPAETH (1936b) described C. ruficornis based on material collected by J. Mráz as he wrote (translated from German): 'So far known only from the state of Sao Paulo, where mainly J. Mráz collected numerous specimens (Holotype and many paratypes in the National Museum in Prague, more paratypes in my collection).' Based on the NMPC material I assume that Spaeth borrowed the series and returned it identified as C. arcuata (Swederus, 1787) as there is no specimen identified as C. ruficornis, and that he retained part of the series for his collection. Later on, when he revised the genus Coptocycla Chevrolat, 1836 he realized that the series collected by J. Mráz actually belonged to a new species, described it and included the NMPC specimens in the type series by indication. However, he failed to label any specimen of the series as 'typus' thus they all must be considered syntypes. The three specimens in his collection have 'cotypus' on their labels (Lech Borowiec, pers. comm. 2020).

In NMPC there are two more specimens of C. ruficornis collected by J. Mráz, however they came from a private collection of A. Jedlička, who received them directly from J. Mráz and thus, they certainly were not examined by F. Spaeth and cannot be part of the type series. 


\section{Coptocycla (Psalidonota) contemta (Boheman, 1855)}

Published records. Piracicaba (Toledo Piza 1968); São Paulo (BoROWIEC 1996).

Distribution. Argentina, Bolivia, Brazil (Mato Grosso, Minas Gerais, Paraná, Rio Grande do Sul, Santa Catarina, São Paulo), Paraguay, and Peru.

\section{Cteisella confusa (Boheman, 1855)}

Published records. São Paulo (Spaeth 1926b); Jundiaí (Borowiec 2002).

Distribution. Argentina, Bolivia, Brazil (Mato Grosso do Sul, Pará, Paraná, Rio Grande do Sul, Rio de Janeiro, Santa Catarina, São Paulo), Paraguay, and Peru.

\section{Cteisella impura (Boheman, 1855)}

Published record. Bocaina (BorowIEC 1996).

Distribution. Brazil (São Paulo).

\section{Cteisella ramosa Spaeth, 1926}

Published record. São Paulo (Spaeth 1926b).

Distribution. Argentina, Brazil (Mato Grosso do Sul, Rio de Janeiro, Rio Grande do Sul, Santa Catarina, São Paulo), and Peru.

\section{Ctenocharidotis crispata (Boheman, 1855)}

Published record. São Paulo (SPAETH 1926b).

Distribution. Brazil (Goiás, Rio Grande do Sul, Santa Catarina, São Paulo).

\section{*Deloyala cruciata (Linnaeus, 1758)}

Published records. Rio Claro; Saltinho (Lopes et al. 2016).

Distribution. Argentina, Bolivia, Brazil (Amazonas, Mato Grosso, Minas Gerais, Pará, Paraná, Rio de Janeiro, Rio Grande do Sul, Santa Catarina, São Paulo), French Guiana, Guyana, Paraguay, Peru, Trinidad and Tobago, and Venezuela.

\section{Eremionycha bahiana (Boheman, 1855)}

Published records. Campinas (BorowIEC 1996); Campinas-Fazenda Santa Elisa, Monjolinho (TeIXEIRA \& CASARI 2003, as Microctenochira difficilis (Boheman, 1855), misidentification).

Distribution. Brazil (Bahia, Espírito Santo, Piauí, Santa Catarina). New to São Paulo.

Remarks. TEIXEIRA \& CASARI (2003) erroneously identified their material as Microctenochira difficilis (Boheman, 1855) while it belongs to E. bahiana (ŚwIĘTOJAŃSKA 2009); however, faunistic record was not adopted (SEKERKA 2020). The species is here registered formally as new to São Paulo. See also Remarks under $M$. difficilis.

\section{*Gratiana conformis (Boheman, 1854)}

Published records. Butantan; Cantareira (BorowIEC 1996); BebedouroFazenda Figueira (CASARI \& TEIXEIRA 2010).
Additional records. BRAZIL: São PAulo: Alto da Serra, 28.ii.1912, 1 spec., G. E. Bryant leg. (BMNH); São Paulo Capital, 20.ii.1912, 1 spec., 24.ii.1912, 5 spec., G. E. Bryant leg. (BMNH).

Distribution. Argentina, Bolivia, Brazil (Paraná, Rio Grande do Sul, Santa Catarina, São Paulo), and Paraguay.

\section{*Gratiana spadicea (Klug, 1829)}

Published record. São Paulo (BorowIEC 1996)

Distribution. Argentina, Brazil (Mato Grosso do Sul, Paraná, Rio Grande do Sul, São Paulo), Paraguay, and Uruguay.

\section{Helocassis flavorugosa (Boheman, 1855)}

New record. BRAZIL: São Paulo: Ilha de Santo Amaro-near Santos, 15.iv.1912, 1 spec., G. E. Bryant leg. (BMNH).

Distribution. Brazil (Paraná, Rio de Janeiro). New to São Paulo.

\section{Hybosa acutangula Spaeth, 1913}

Published record. Teodoro Sampaio (BorowIEC 1996).

Distribution. Argentina, Brazil (Espírito Santo, Rio Grande do Sul, Paraná, Santa Catarina, São Paulo), and Paraguay.

\section{Hybosa fornicata Boheman, 1855}

Published records. Rio Paraná (Borowiec 1996); Ourinho; São José do Rio Preto (BorowIEC 2002)

Distribution. Bolivia, Brazil (Goiás, São Paulo), and Paraguay.

\section{*Metriona elatior (Klug, 1829)}

Published records. Barueri; Butantan; Campos de Jordão (BorowIEC 1996); Campinas (Simões \& Monné 2008); Jundiaí (BorowieC \& TAKIZAWA 2011).

Additional records. BRAZIL: São Paulo: São Paulo Capital, 18.ii.1912, 1 spec., 21.ii.1912, 1 spec., G. E. Bryant leg. (BMNH); Serra de Bocaina, 1500 m, xi.1965, 2 spec. (NHMB).

Distribution. Argentina, Brazil (Goiás, Minas Gerais, Paraná, Rio de Janeiro, Rio Grande do Sul, Santa Catarina, São Paulo), Paraguay, and Uruguay.

Remarks. BOROWIEC (1996) recorded several species, which are common in southern part of South America, as new to French Guiana based on old material labelled as from Bas Maroni. BorowiEC \& MORAGUES (2005) critically evaluated all records published from French Guiana and removed four of these species published from Bas Maroni as their occurrence in French Guiana is very improbable; however, M. elatior remained in the review as an accepted record. In my opinion, also the specimens of $M$. elatior were mislabelled as the species occurs mainly in the Paraná Basin and along the Brazilian coast reaches as north as Rio de Janeiro. Also, the record from the state of Pará (Santarém) is dubious as M. elatior is usually associated with seasonally dry or semi-dry savannas and semideciduous forests. Moreover, $M$. elatior is one of the most common species of Brazilian cassidines, collected usually by almost every collector who collected some cassidines, and from each of 
the other states it has been confirmed by many specimens from multiple localities. Indeed, the present-day knowledge of the distribution of cassidines in Brazil is still far from being complete but based on field experience and general distribution patterns of cassidines, I find these two records very improbable and thus remove Pará and French Guiana from the distribution of $M$. elatior.

\section{*Metrionella calva (Boheman, 1855)}

New record. BRAZIL: São PAULo: without further data, 3 spec., J. Mráz leg. (NMPC, 1 LSPC).

Distribution. Bolivia, Brazil, Paraguay, and Venezuela. New to São Paulo.

Remarks. One of the abovementioned specimens is provided with the following identification label: 'Metriona placans m. [hw] | Spaeth det. | n. sp. [hw] | (Metrionella) $[\mathrm{hw}]$ | n. subg. [hw] [white printed label with some rows handwritten (hw) by Spaeth]'; however, Spaeth did not mention this specimen in the description of $M$. placans thus it does not belong to the type series. Moreover, it differs quite conspicuously from M. placans in more convex elytra with a distinct postscutellar hump (absent in M. placans), clypeal grooves deep and enclosing a short triangular field reaching only middle of the clypeus (vs. shallow grooves enclosing a long field reaching almost the base of antennae), and thorax black (vs. mesoventrite with large yellow patch on each side). These characters seem to match quite exactly M. calva, which so far has been reported from Brazil without additional data, despite the fact that SPAETH (1932b) mentioned that it is not a rare species in the tropical America. However, he did not mention its distribution more precisely.

\section{*Microctenochira aciculata (Boheman, 1855)}

Published records. Cantareira (Borowiec 1996); Mogi das CruzesFazenda Carmelita (BorowIEC 2002); Rio Claro (LoPES et al. 2016).

Distribution. Argentina, Brazil (Bahia, Paraná, Rio de Janeiro; Santa Catarina, São Paulo), and Paraguay.

\section{Microctenochira brasiliensis Świętojańska \& Borowiec, 1999}

Published records. Barueri (ŚwiętojańSKa \& Borowiec 1999); Serra do Japi-Jundiaí (Buzzi 1999, as M. frieirocostai Buzzi, 1999).

Distribution. Brazil (São Paulo).

\section{Microctenochira circumcincta (Boheman, 1855)}

Published record. São Paulo (SPAETh 1926b).

Distribution. Brazil (Bahia, São Paulo).

\section{*Microctenochira optata (Boheman, 1855)}

Published records. Barueri; Cantareira (BorowIEC 1996); Rio Claro; Saltinho (Lopes et al. 2016).

Distribution. Argentina, Bolivia, Brazil (Alagoas, Bahia, Goiás, Mato Grosso, Minas Gerais, Pará, Paraná, Rio de Janeiro, Rio Grande do Sul, Santa Catarina, São Paulo), French Guiana, Paraguay, and Peru.
*Microctenochira patruelis (Boheman, 1855)

New records. BRAZIL: São Paulo: without further data, 1 spec., J. Mráz leg. (NMPC); Alto da Serra, 5.iii.1912, 1 spec., 16.iii.1912, 1 spec., G. E. Bryant leg. (BMNH)

Distribution. Brazil (Espírito Santo, Goiás, Minas Gerais, Rio de Janeiro). New to São Paulo.

\section{Microctenochira quadrata (DeGeer, 1775)}

Published records. Rio Claro; Saltinho (Lopes et al. 2016).

Distribution. Brazil (Goiás, Mato Grosso, Minas Gerais, Pará, Santa Catarina, São Paulo), Colombia, French Guiana, Guyana, Paraguay, Suriname, Trinidad and Tobago, and Venezuela.

\section{*Microctenochira similata (Boheman, 1855)}

Published records. São Paulo (BoHEMan 1855, as M. sellata (Boheman, 1855)); São Paulo; 'Apiaty [= Apiaí]' (SPAETH 1926b).

Distribution. Argentina, Brazil (Rio Grande do Sul, Rio de Janeiro, Santa Catarina, São Paulo), and Paraguay.

\section{*Microctenochira triplagiata (Spaeth, 1926)} (Fig. 4)

Published record. São Paulo (SPAETh 1926b).

Type material examined. HolOTYPe (Fig. 4): pinned, 'SAÕ PAULO | BRAS. MRÁZ LGT. | MUS.PRAGENSE [w, p, cb, bf] || TYPUS [r, p, cb, bf] || Ctenochira | triplagiata m. [hw by F. Spaeth] | Spaeth det. | Typ. unic. [hw by F. Spaeth] [w, p, cb]' (NMPC).

Distribution. Brazil (São Paulo).

\section{Plagiometriona approximans Spaeth, 1937}

Published record. Alto da Serra (SPAETh 1937).

Distribution. Brazil (São Paulo).

*Plagiometriona constricta (Boheman, 1855)

Published record. Campos do Jordão (BoROwIEC 1996).

Distribution. Brazil (São Paulo).

*Plagiometriona deyrollei (Boheman, 1855)

New record. BRAZIL: São Paulo: without further data, 3 spec., J. Mráz leg. (NMPC).

Distribution. Brazil (Santa Catarina). New to São Paulo.

\section{Plagiometriona dorsosignata (Boheman, 1855)}

Published record. Campos do Jordão (BorowIEC 1996). Additional records. BRAZIL: SÃo PAulo: Alto da Serra, 9.iii.1912, 2 spec., G. E. Bryant leg. (BMNH); Serra de Bocaina, 1500 m, xi.1965, 1 spec. (NHMB).

Distribution. Brazil (Rio de Janeiro, São Paulo).

\section{Plagiometriona flavescens (Boheman, 1855)}

Published records. São Paulo (BoROwIEC 1996); Parque Estadual Intervales (NogueIRA-DE-SÁ \& TRIGo 2002, 2005); Ubatuba (BorowIEC 2009). Additional records. BRAZIL: São Paulo: Santos, v.1883, 1 spec. (BMNH), 22.ix.1911, 1 spec., 22.ii.1912, 2 spec., 1.iii.1912, 1 spec., 2.iii.1912, 5 spec., 29.iv.1912, 1 spec., G. E. Bryant leg. (BMNH) 
Distribution. Argentina, Brazil (Mato Grosso, Rio de Janeiro, São Paulo), and Paraguay.

\section{Plagiometriona forcipata (Boheman, 1855)}

Published records. Campos do Jordão (BorowIEC 1996, also under $P$. emarcida (Boheman, 1855)).

Distribution. Brazil (Rio de Janeiro, São Paulo).

*Plagiometriona praecincta (Boheman, 1855)

Published records. Piracicaba (Toledo PIZA 1968); São Paulo (BorowIEC 1996).

Distribution. Brazil (Rio de Janeiro, Santa Catarina, São Paulo).

\section{*Plagiometriona punctatissima (Boheman, 1855)}

Published record. Jundiaí (BorowIEC 2002, as P. punctipennis [sic!] (Boheman, 1855)).

Distribution. Argentina and Brazil (Paraná, Santa Catarina). New to São Paulo.

Remarks. BOROWIEC (2002) published a record under the name Plagiometriona punctipennis (Boheman, 1855) due to a mistake in spelling, which in fact belongs to P. punctatissima (Lech Borowiec, pers. comm. 2020). Therefore, the species is here recorded from São Paulo for the first time. The entry Plagiometriona punctipennis listed in the catalogue (BorowIEC 1999: 408) must be deleted as it is a duplicate of Agroiconota punctipennis (Boheman, 1855).

\section{*Plagiometriona stillata (Boheman, 1855)}

Published records. Cubatão; Santo André (Paranapiacaba) (SIMõES \& MonNÉ 2008).

Distribution. Brazil (Espírito Santo, Rio de Janeiro, Santa Catarina, São Paulo).

\section{*Plagiometriona tenella (Klug, 1829)}

New record. BRAZIL: São Paulo: without further data, 10 spec., J. Mráz leg. (NMPC).

Distribution. Argentina, Brazil (Paraná, Rio Grande do Sul, Santa Catarina), and Paraguay. New to São Paulo.

\section{*Plagiometriona vigens (Boheman, 1855)}

Published records. Bocaina; Cantareira (BorowIEC 1996); Paranapiacaba (BOROWIEC 2009).

Additional record. BRAZIL: São PAulo: Serra de Bocaina, 1500 m, xi.1965, 1 spec. (NHMB).

Distribution. Brazil (Minas Gerais, Rio de Janeiro, Rio Grande do Sul, São Paulo).

\section{Tribe Chalepini \\ *Acritispa dilatata (Uhmann, 1932)}

Published records. Mato do Governo; Fazenda Pau d'Alho (Uhmann 1964); São Paulo (STAINES 1988).

Distribution. Brazil (Bahia, Minas Gerais, Paraíba, São Paulo) and Paraguay.

\section{Anisostena (s. str.) promta Weise, 1910}

Published records. São Paulo (Weise 1910b, StaINes 1994); Bocaina (UHMANN 1932).

Distribution. Argentina, Brazil (Bahia, Minas Gerais, Mato Grosso, Rio de Janeiro, São Paulo), and Paraguay.

\section{*Anisostena (Apostena) angustata Pic, 1934}

Published record. São Paulo Capital-Rua Maestro Cardim (Uhmann 1964).

Distribution. Argentina and Brazil (Rio Grande do Sul, Santa Catarina, São Paulo).

\section{*Baliosus conspersus Weise, 1911}

New records. BRAZIL: São PAulo: without further data, 1 spec., J. Mráz leg. (NMPC); Jundiaí, 750 m, 1.-15.xi.1976, 1 spec., A. B. Schneble leg. (SMNS).

Distribution. Argentina, Brazil (Santa Catarina), and Paraguay. New to São Paulo.

\section{Baliosus hospes Weise, 1905}

Published records. Jabaquara; São Paulo Capital (Uhmann 1964).

Distribution. Bolivia, Brazil (Goiás, Rio Grande do Sul, São Paulo), and Paraguay.

*Baliosus parvulus (Chapuis, 1877)

Published records. Bocaina (Uhmann 1932), Fazenda Pau d'Alho (UHMANN 1964).

Additional records. São Paulo: Jundiaí, 750 m, 1.-15.xi.1976, 3 spec., A. B. Schneble leg. (SMNS, 1 LSPC).

Distribution. Argentina, Brazil (Bahia, Mato Grosso, Minas Gerais, Rio Grande do Sul, Rio de Janeiro, Santa Catarina, São Paulo), and Paraguay.

\section{*Baliosus pectoralis (Baly, 1864)}

Published record. Cantareira (UHMANN 1964)

Distribution. Brazil (São Paulo).

\section{Baliosus rubiginosus (Guérin-Méneville, 1844)}

Published record. Campinas (Uhmann 1939).

Distribution. Argentina, Bolivia, Brazil (Goiás, Mato Grosso, Rio Grande do Sul, Santa Catarina, São Paulo), Paraguay, and Venezuela.

\section{*Baliosus subparvulus Uhmann, 1948}

Published records. Alto da Serra; Bosque de Saúde (Uhmann 1948b), Salesópolis-Estação Biológica de Boraceia (UHMANN 1964).

Distribution. Brazil (Minas Gerais, São Paulo).

\section{Baliosus terminatus (Chapuis, 1877)}

Published records. Barueri; Jabaquara (Uhmann 1964).

Distribution. Brazil (Santa Catarina, São Paulo). 


\section{Baliosus varius Weise, 1911}

Published records. Santos (WeISE 1921); São Paulo Capital-Jabaquara (UHMANN 1964).

Distribution. Brazil (Mato Grosso, São Paulo).

\section{Bothrispa depressa (Chapuis, 1877)}

Published records. Batataes; Itu-Fazenda Pau d'Alho; Jundaí (UhmanN 1964).

Distribution. Brazil (Goiás, Mato Grosso, Minas Gerais, São Paulo).

\section{Bruchia sparsa Weise, 1906}

Published record. São Bernardo do Campo (Uhmann 1964).

Distribution. Argentina, Brazil (Bahia, Minas Gerais, Pará, São Paulo), and Ecuador.

Chalepotatus (s. str.) coarctatus (Chapuis, 1877)

Published record. Monte Alegre (Uhmann 1964).

Distribution. Argentina, Brazil (Rio Grande do Sul, Santa Catarina, São Paulo), and Paraguay.

\section{*Chalepus aenescens Weise, 1910}

New record. BRAZIL: São PAulo: without further data, 1 spec., J. Mráz leg. (NMPC)

Distribution. Brazil (Santa Catarina). New to São Paulo.

\section{Chalepus consimilis Weise, 1905}

Published record. Porto Epitácio (Uhmann 1964).

Distribution. Argentina, Brazil (Mato Grosso, Santa Catarina, São Paulo), and Paraguay.

\section{*Chalepus cordiger (Chapuis, 1877)}

Published records. São Paulo (Uhmann 1938b); Ilha Seca (SouzA Lopes 1940); Cantareira; Estação Biologica de Paranapiacaba; ItuFazenda Pau d'Alho; São Paulo Capital (Uhmann 1964).

Distribution. Argentina, Brazil (Bahia, Minas Gerais, Rio Grande do Sul, Santa Catarina, São Paulo), and Paraguay.

\section{Chalepus dorni Uhmann, 1930}

Published record. Campinas (Uhmann 1936).

Distribution. Brazil (Minas Gerais, São Paulo).

\section{Chalepus erosus Uhmann, 1948}

Published record. Campos do Jordão (Uhmann 1964)

Distribution. Brazil (Minas Gerais, Santa Catarina, São Paulo).

\section{*Chalepus marginiventris (Chapuis, 1877)}

Published records. São Paulo (Uhmann 1931); Bocaina (Uhmann 1932); Boraceia (Buck 1958); Barueri; Cantareira; Cocaia; Estação Biológica de Paranapiacaba; São Paulo Capital (Uhmann 1964). Additional record. BRAZIL: São Paulo: Jundiaí, $750 \mathrm{~m}, 1 .-15$. xi.1976, 4 spec., A. B. Schneble leg. (SMNS, 1 LSPC).

Distribution. Argentina and Brazil (Bahia, Goiás, Mato
Grosso, Minas Gerais, Paraná, Rio de Janeiro, Rio Grande do Sul, Santa Catarina, São Paulo).

\section{Chalepus pauli Pic, 1932}

Published record. São Paulo (Descarpentries \& Villiers 1959).

Distribution. Brazil (São Paulo).

Remarks. In the original description, PIC (1932) mentioned that the species is from 'Brésil' only; however, the type material also has São Paulo written on the labels, which was specified by Descarpentries \& Villiers (1959).

\section{*Chalepus quadricostatus (Chapuis, 1877)}

Published record. Bocaina (UHMANN 1932)

Distribution. Argentina, Brazil (Rio Grande do Sul, Rio de Janeiro, Santa Catarina, São Paulo).

\section{*Chalepus sanguinicollis (Linnaeus, 1771)}

Published records. Ilha Seca (Souza Lopes 1940); Anhangai; Barueri; Ilha Bela; Indiana; Iporanga; Itu-Fazenda Pau d'Alho; São Paulo-Rua Maestro Cardim (Uhmann 1964).

Additional record. BRAZIL: São Paulo: Jundiaí, $750 \mathrm{~m}, 1 .-15$. xi.1976, 3 spec., A. B. Schneble leg. (SMNS).

Distribution. Argentina, Bolivia, Brazil (Amapá, Bahia, Espírito Santo, Goiás, Mato Grosso, Paraná, Rio Grande do Sul, Santa Catarina, São Paulo), Colombia, Cuba, Dominican Republic, French Guiana, Jamaica, Paraguay, Puerto Rico, Suriname, Trinidad and Tobago, and United States of America.

\section{Charistena ruficollis (Fabricius, 1801)}

Published records. São Paulo (Weise 1921, Uhmann 1938c); Ilha Seca (Souza Lopes 1940); Barueri; Ilha Bela; Itu-Fazenda Pau d'Alho; Salesópolis-Estação Biológica de Boraceia; São Paulo-Rua Maestro Cardim; São Sebastião-Barra de Una (Uhmann 1964).

Additional record. BRAZIL: São PAulo: Jundiaí, $750 \mathrm{~m}, 1 .-15$. xi.1976, 14 spec., A. B. Schneble leg. (SMNS, 2 LSPC).

Distribution. Argentina, Bolivia, Brazil (Bahia, Minas Gerais, Pernambuco, Rio de Janeiro, Santa Catarina, São Paulo), Colombia, Costa Rica, French Guiana, Panama, Suriname, and Venezuela.

\section{Clinocarispa plaumanni Uhmann, 1938}

Published records. Ilha Santo Amaro near Santos (Uhmann 1938); Jacupiranga (RAMOs 1996).

Distribution. Brazil (Santa Catarina, São Paulo).

\section{Clinocarispa sauveuri (Chapuis, 1877)}

Published records. Araçatuba-Rio Jacaretinga; Boa Esperança do Sul; São Paulo (Ramos 1996).

Distribution. Brazil (Amazonas, Bahia, Espírito Santo, Goiás, Minas Gerais, Rio de Janeiro, Santa Catarina, São Paulo), Colombia, and Peru.

Corynispa clavicornis (Uhmann, 1930)

Published record. Saúde (Uhmann 1937a).

Distribution. Brazil (Bahia, Rio de Janeiro, Sao Paulo). 


\section{Decatelia pallipes (Weise, 1922)}

New records. BRAZIL: São PAulo: Ribeirão Pires, xi.1898, 1 spec., E. Gounelle leg. (MNHN); Vale do Rio Prado, xii.1898, 1 spec., E. Gounelle leg. (MNHN).

Distribution. Argentina, Brazil (Minas Gerais, Rio de Janeiro, Santa Catarina), and Paraguay. New to São Paulo.

\section{Heptachispa crassicornis (Chapuis, 1877)}

Published record. Itu-Fazenda Pau d'Alho (Uhmann 1964).

Distribution. Brazil (Goiás, Minas Gerais, São Paulo).

\section{Heptachispa delkeskampi (Uhmann, 1940)}

Published record. Barueri (Uhmann 1964).

Distribution. Brazil (Amazonas, Mato Grosso, São Paulo) and Paraguay.

\section{Heptatomispa kesseli Uhmann, 1932}

Published record. Bocaina (UHMANN 1932).

Distribution. Brazil (São Paulo).

Remarks. Uhmann (1932) described the genus Heptatomispa with a single species $H$. kesseli but formally did not mention that it is the type of the genus. Later on, UHMANN (1940) published a list of type species for the genera he established in his earlier publications without original designation of the type species. Subsequently, UHMANN (1957) listed the generic name with year 1940 and considered its 1932 appearance a nomen nudum; this was followed until recently (e.g., STAINES 2002). However, UHMANN (1932) combined description of a new genus and a single new nominal species assigned to it and provided them with 'n. gen.' and 'n. sp.', respectively, thus conditions of the Article 13.4. are fulfilled and the name is valid from the 1932 description (ICZN 1999).

\section{*Heterispa costipennis (Boheman, 1859)}

Published records. Santos (Weise 1906, as H. orientalis Weise, 1906); São Paulo (Uhmann 1931); Barueri; Cantareira; Cubatão; Diadema; Iporanga; Itu-Pau d'Alho; São Paulo Capital (Uhmann 1964); São Paulo-Ipiranga (CASARi \& Teixeira 2004, as H. vinula (Erichson, 1847)).

Additional records. São Paulo: Jundiaí, 750 m, 1.-15.xi.1976, 6 spec., A. B. Schneble leg. (SMNS).

Distribution. Argentina, Bolivia, Brazil (Bahia, Espírito Santo, Minas Gerais, Paraná, Rio Grande do Sul, Santa Catarina, São Paulo), ? Ecuador, Paraguay, and Uruguay.

Remarks. Species of Heterispa Chapuis, 1875 are hard to identify as they are very similar to each other and the widespread species are rather variable with some local forms. The genus was never revised, and the identification of specimens is rather putative. Usually, all specimens with predominantly dark elytra with only humeri pale coloured are identified as $H$. vinula (Erichson, 1847). However, the mentioned species is distributed along the Andes, and in southern Bolivia and Argentina it is replaced by $H$. costipennis distributed in the Paraná Basin and central and eastern Brazil.

CASARI \& TEIXEIRA (2004) published a description of larva which was identified as $H$. vinula. Based on the photograph of the adult available in the same publication, the description certainly belongs to $H$. costipennis and thus $H$. vinula does not occur in São Paulo.

\section{Metazycera purpurata (Guérin-Méneville, 1844)}

Published record. Campinas (CASARI 2005).

Distribution. Brazil (Espírito Santo, Rio de Janeiro, São Paulo), Colombia, French Guiana, Paraguay, Suriname, and Uruguay.

\section{Octhispa binotata (Chapuis, 1877)}

Published records. São Paulo (Uhmann 1939); Cantareira (Uhmann 1964).

Distribution. Brazil (Bahia, Minas Gerais, São Paulo) and Paraguay.

\section{Octhispa elongata (Chapuis, 1877)}

Published records. Anhangaí; São Paulo-Ipiranga (Uhmann 1964).

Distribution. Argentina, Bolivia, Brazil (Goiás, Mato Grosso, Pernambuco, Rio Grande do Sul, Santa Catarina, São Paulo), and Paraguay.

\section{*Octhispa gemmata (Germar, 1823)}

New record. BRAZIL: São Paulo: without further data, 1 spec., J. Mráz leg. (NMPC).

Distribution. Argentina, Brazil (Goiás, Rio Grande do Sul, Santa Catarina), and Paraguay. New to São Paulo.

\section{Octhispa kraatzi Weise, 1905}

Published record. Santos (WeISE 1905).

Distribution. Brazil (São Paulo), French Guiana, and Suriname.

\section{Octhispa pustulata (Chapuis, 1877)}

Published records. Jabaquara; São Paulo Capital (Uhmann 1964).

Distribution. Brazil (Bahia, São Paulo).

\section{Octhispa quadrinotata Weise, 1905}

Published record. Cantareira (Uhmann 1964).

Distribution. Brazil (Goiás, Minas Gerais, São Paulo) and Peru.

\section{*Octhispa robinsonii (Baly, 1864)}

New record. BRAZIL: São PAulo: without further data, 3 spec., J. Mráz leg. (NMPC).

Distribution. Brazil (Espírito Santo). New to São Paulo.

\section{Octhispa severini Weise, 1911}

Published records. Barueri; Rio Grande-Repreza near São Paulo Capital (UHMANN 1964).

Distribution. Brazil (Espírito Santo, Goiás, Rio Grande do Sul, Rio de Janeiro, Santa Catarina, São Paulo) and Paraguay. 


\section{Octhispa spitzi Uhmann, 1938}

Published records. Jabaquara; São Paulo Capital (Uhmann 1964).

Distribution. Argentina, Brazil (Bahia, Minas Gerais, Santa Catarina, São Paulo), and Paraguay.

\section{*Octotoma brasiliensis Weise, 1921}

New record. BRAZIL: São PAulo: without further data, 4 spec., J. Mráz leg. (NMPC, 1 LSPC).

Distribution. Brazil. New to São Paulo.

Remarks. The species was so far known only from the type material labelled generally Brazil.

\section{*Octotoma crassicornis Weise, 1910}

New record. BRAZIL: São PAulo: without further data, 2 spec., J. Mráz leg. (NMPC, LSPC).

Distribution. Brazil (Minas Gerais). New to São Paulo.

\section{Octuroplata walkeri (Baly, 1865)}

Published record. Salesópolis-Estação Biológica de Boracéia (CASARI \& QueIroz 2005).

Distribution. Argentina, Brazil (Minas Gerais, Santa Catarina, São Paulo), and Paraguay.

\section{Oxychalepus anchora (Chapuis, 1877)}

Published records. Jabaquara; $10 \mathrm{mi}$ W of Sorocaba (STAINES 2010).

Distribution. Argentina, Belize, Bolivia, Brazil (Bahia, Distrito Federal, Goiás, Mato Grosso do Sul, Rondônia, São Paulo), Colombia, Costa Rica, Ecuador, El Salvador, Guatemala, Honduras, Mexico, Nicaragua, Panama, Paraguay, Peru, Trinidad and Tobago, Venezuela.

Remarks. Ramos (1998) revised the Brazilian species of Oxylepus and excluded O. anchora from the fauna of Brazil as the records published earlier belonged to O. externus. Oxychalepus anchora is a species very common and widespread in Central America and NE portion of South America but the records from the southern part of South America are dubious and must be verified. In general, species of Oxychalepus Uhmann, 1937 are quite difficult to identify due to their morphological similarity and strong polymorphism.

STAINES (2010) published a revision of the entire genus which, however, contains numerous mistakes and misidentifications, and many specimens fall under several different species using the key he proposed; therefore, a great part of the records must be considered with caution. In the same revision, $O$. anchora was published from two localities in São Paulo.

\section{*Oxychalepus centralis Uhmann, 1940}

New record. BRAZIL: São Paulo: without further data, 1 spec., J. Mráz leg. (NMPC)

Distribution. Argentina, Brazil (Amazonas, Mato Grosso, Minas Gerais, Rio de Janeiro, Rondônia), and Paraguay. New to São Paulo.

\section{*Oxychalepus externus (Chapuis, 1877)}

Published records. Itu-Fazenda Pau d'Alho; São Paulo-Ipiranga (Uhmann 1964, also as O. confinis Weise, 1911); Atibaia; Barueri; Caraguatatuba; Itu-Fazenda Cuiabá; Itu-Fazenda Pau d'Alho; Jundiaí; Mairiporã; Monte Alegre-Fazenda Experim.; Ona Verde-Fazenda São João; Riberão Preto; São Paulo-Ipiranga (RAmos 1998); Campinas; Cantareira; Horto Florestal; São Paulo (StAInes 2010).

Distribution. Argentina, Bolivia, Brazil (Amazonas, Bahia, Espiríto Santo, Goiás, Mato Grosso, Mato Grosso do Sul, Minas Gerais, Pará, Paraíba, Pernambuco, Rio de Janeiro, Rio Grande do Sul, Rondônia, Santa Catarina, São Paulo), Ecuador, French Guiana, Paraguay, Peru, Suriname, and Venezuela.

\section{Oxychalepus insignitus (Chapuis, 1877)}

Published records. Barueri (Uhmann 1964); Santos (Staines 2010).

Distribution. Brazil (Goiás, Minas Gerais, Paraná, São Paulo).

Remarks. Ramos (1998) synonymized this taxon with O. proximus; however, STAINES (2010) restored its species status. The original specimen from Barueri was also studied and published again by RAmos (1998) as $O$. proximus. The validity of this taxon is a subject of further revision.

\section{Oxychalepus proximus (Guérin-Méneville, 1844)}

Published record. Nova Europa-Fazenda Itaquerê (Ramos 1998).

Distribution. Bolivia, Brazil (Goiás, Mato Grosso, Mato Grosso do Sul, Pará, Rio Grande do Sul, Santa Catarina, São Paulo, Tocantins), and Paraguay.

\section{Physocoryna parvula Weise, 1921}

Published record. São Paulo (STAINES 1998).

Distribution. Brazil (Bahia, São Paulo) and Paraguay.

\section{Physocoryna scabra Guérin-Méneville, 1844}

Published records. Barueri; Itanhaém (Uhmann 1964).

Distribution. Argentina, Brazil (Bahia, Goiás, Mato Grosso, Pará, Rio Grande do Sul, Rio de Janeiro, Santa Catarina, São Paulo), Colombia, Paraguay, and Peru.

Remarks. Material from Itanhaém published by (UHMANN 1964) was mentioned again by STAINES (1998: 167) but wrongly assigned to the state of Pará.

\section{*Probaenia crenata (Blanchard, 1843)}

Published record. Itu-Fazenda Pau d'Alho (Uhmann 1964). Additional record. BRAZIL: São Paulo: Amparo, 4.-30.iv.1982, 1 spec., Wewalka leg. (NHMW).

Distribution. Argentina, Bolivia, Brazil (Goiás, Minas Gerais, Mato Grosso, Paraná, Rio Grande do Sul, Rio de Janiero, São Paulo), Chile, Colombia, and Paraguay.

\section{Probaenia decipiens (Chapuis, 1877)}

Published record. Bosque de Saúde (Uhmann 1948b).

Distribution. Brazil (São Paulo). 


\section{*Probaenia grayi (Baly, 1865)}

Published records. Aracatuba; Barueri; Campos do Jordão; Cantareira; Interlagos; Itu-Fazenda Pau d'Alho (Uhmann 1964).

Additional record. BRAZIL: São Paulo: Jundiaí, $750 \mathrm{~m}, 1 .-15$ xi.1976, 2 spec., A. B. Schneble leg. (SMNS).

Distribution. Argentina, Brazil (Bahia, Minas Gerais, Paraná, Rio de Janeiro, Rio Grande do Sul, Santa Catarina, São Paulo), and Paraguay.

\section{*Probaenia nobilis (Chapuis, 1877)}

Published records. Barueri; Interlagos; Salesópolis-Estação Biológica de Boraceia; São Paulo-Ipiranga (Uhmann 1964).

Distribution. Brazil (Bahia, Rio de Janeiro, Santa Catarina, São Paulo).

\section{Probaenia weisei Uhmann, 1927}

Published record. Santos (Uhmann 1927).

Distribution. Brazil (São Paulo).

\section{*Sternostena triangularis Uhmann, 1931}

Published records. São Paulo (Uhmann 1931); Bocaina (Uhmann 1932, as $S$. varians ab. triangularis Uhmann, 1932); Cidade Universitária; São Paulo Capital (Uhmann 1964).

Distribution. Brazil (São Paulo).

Remarks. UhMANN (1931) described S. triangularis. A year later, UHMANN (1932) described $S$. varians ab. triangularis. According to the catalogue (UHMANN 1957), both nominal taxa belong to the same species and moreover, the aberration is an unavailable infrasubspecific name.

\section{Sumitrosis brevenotata (Pic, 1929)}

Published record. São Paulo (DesCARPENTRIES \& Villiers 1959).

Distribution. Brazil (São Paulo).

Remarks. In the original description, PIC (1929) mentioned 'Brésil' only; however, the type material also has São Paulo written on the labels, which was specified by DESCARPENTRIES \& VilLIERS (1959).

\section{Sumitrosis difficilis (Monrós \& Viana, 1947)}

Published record. George Oeterer (Uhmann 1964).

Distribution. Argentina, Bolivia, and Brazil (Goiás, São Paulo).

\section{Sumitrosis fuscicornis (Weise, 1910)}

Published record. São Paulo (MonRós \& VIANA 1947).

Distribution. Argentina, Brazil, (Bahia, Santa Catarina, São Paulo), and Colombia.

Remarks. MONRÓs \& VIANA (1947) mentioned occurrence of S. fuscicornis in São Paulo and attributed the record to BosQ (1943). However, the occurrence in Brazil is not mentioned in the latter publication. Monrós \& Viana were not particularly clear when mentioning the source of distributional information; sometimes it referred to a publication while in other cases to a collection from which they examined material. I assume that the latter is also the case here and they may have studied material from the collection of Bosq.

\section{Sumitrosis maculata (Uhmann, 1931)}

Published record. São Paulo (Uhmann 1931).

Distribution. Brazil (São Paulo).

Sumitrosis reichardti (Uhmann, 1968)

Published record. Magda (Uhmann 1968).

Distribution. Brazil (São Paulo).

Temnochalepus imitans Uhmann, 1935

New record. BRAZIL: São Paulo: Jundiaí, 750 m, 1.-15.xi.1976, 3 spec., A. B. Schneble leg. (SMNS, 1 LSPC).

Distribution. Brazil (Rio de Janeiro). New to São Paulo.

*Temnochalepus insolitus Uhmann, 1935

Published records. Anhangai; Indiana (Uhmann 1964).

Distribution. Argentina, Bolivia, Brazil (Mato Grosso, Paraná, Rio Grande do Sul, Santa Catarina, São Paulo), and Paraguay.

\section{Temnochalepus lugubris (Chapuis, 1877)}

Published record. São Roque (Uhmann 1964).

Distribution. Argentina, Brazil (Amazonas, Bahia, Goiás, Pernambuco, Rio de Janeiro, Santa Catarina, São Paulo), and Paraguay.

\section{Uroplata acuta Uhmann, 1968}

Published record. Aracatuba-Rio Jacarecatinga (UHMANn 1968).

Distribution. Brazil (Mato Grosso do Sul, São Paulo).

\section{Uroplata bilineata Chapuis, 1877}

Published record. São Paulo (MonRós \& VianA 1947).

Distribution. Argentina, Bolivia, Brazil (Bahia, São Paulo), and Paraguay.

\section{Uroplata borgmeieri Uhmann, 1937}

Published record. Serro Azul (Uhmann 1937b).

Distribution. Brazil (Rio Grande do Sul, São Paulo).

*Uroplata coarctata Weise, 1921

New record. BRAZIL: São Paulo: without further data, 1 spec., J. Mráz leg. (NMPC).

Distribution. Argentina, Brazil (Bahia, Distrito Federal, ? Mato Grosso, Minas Gerais, Rio de Janeiro, Rio Grande do Sul), and Paraguay. New to São Paulo.

\section{*Uroplata ferruginea Weise, 1905}

Published record. São Paulo (Uhmann 1931).

Distribution. Brazil (Goiás, São Paulo). 
Uroplata girardi Pic, 1934

Published record. São Paulo (Uhmann 1937b).

Distribution. Argentina, Brazil (Bahia, Minas Gerais, Rio Grande do Sul, Santa Catarina, São Paulo), and Paraguay.

\section{Uroplata iheringi Weise, 1911}

Published record. Campos do Jordão (WeISE 1911).

Distribution. Brazil (São Paulo).

\section{Uroplata insularum Uhmann, 1968}

Published records. Ilha dos Búzios; Ilha da Vitória (Uhmann 1968).

Distribution. Brazil (São Paulo).

\section{Uroplata interrupta Weise, 1911}

Published records. Barueri; Jabaquara (Uhmann 1964).

Distribution. Brazil (Minas Gerais, São Paulo).

\section{*Uroplata minuscula (Chapuis, 1877)}

New record. BRAZIL: São Paulo: without further data, 2 spec., J. Mráz leg. (NMPC).

Distribution. Argentina, Brazil (Rio Grande do Sul), Paraguay, and Uruguay. New to São Paulo.

\section{Uroplata reimoseri Spaeth, 1937}

Published records. Barueri; Itapecarica (Uhmann 1964).

Distribution. Brazil (São Paulo) and Paraguay.

*Xenochalepus (s. str.) bicostatus (Chapuis, 1877)

Published record. Franca (Uhmann 1964).

Distribution. Argentina, Brazil (Goiás, Mato Grosso, Rio de Janeiro, Rio Grande do Sul, Santa Catarina, São Paulo), Paraguay, and Venezuela.

\section{Xenochalepus (Neochalepus) assimilis Uhmann, 1938}

Published records. Saúde (Uhmann 1938a); Itanhaém; Ilha de São Sebastião (Uhmann 1964).

Distribution. Brazil (Bahia, Rio de Janeiro, Rio Grande do Sul, Santa Catarina, São Paulo).

\section{Xenochalepus (Neochalepus) dictiopterus (Perty, 1832)}

Published records. Barueri; Itu-Fazenda Pau d'Alho (Uhmann 1964).

Distribution. Brazil (Amazonas, Santa Catarina, São Paulo).

Xenochalepus (Neochalepus) guerini (Chapuis, 1877)

Published records. São Paulo (Uhmann 1931, 1938a); Bosque da Saúde; Porto Epitácio (Uhmann 1964)

Distribution. Argentina, Bolivia, Brazil (Bahia, Espírito Santo, Goiás, Mato Grosso, Rio de Janeiro, Santa Catarina, São Paulo), Paraguay, and Peru.
*Xenochalepus (Neochalepus) monrosi Uhmann, 1951

Published records. Fazenda do Bonito; São José do Barreiro; Serra da Bocaina (UHMANN 1964).

Distribution. Brazil (Paraná, Rio Grande do Sul, São Paulo).

*Xenochalepus (Neochalepus) platymeroides Uhmann, 1938

Published record. São Paulo-Ipiranga (Uhmann 1964).

Distribution. Brazil (Bahia, Minas Gerais, Rio de Janiero, Santa Catarina, São Paulo).

*Xenochalepus (Neochalepus) platymerus (Lucas, 1859)

Published record. Campos do Jordão (Uhmann 1964)

Distribution. Brazil (Minas Gerais, Rio Grande do Sul, Rio de Janeiro, Santa Catarina, São Paulo) and Paraguay.

\section{*Xenochalepus (Neochalepus) trilineatus (Chapuis, 1877)}

Published records. Diadema; São Paulo-Rua Maestro Cardim; Rio Grande-Repreza near São Paulo Capital (Uhmann 1964).

Additional record. BRAZIL: São Paulo: Jundiaí, $750 \mathrm{~m}, 1 .-15$. xi.1976, 1 spec., A. B. Schneble leg. (SMNS).

Distribution. Argentina and Brazil (Bahia, Goiás, Mato Grosso, Rio de Janeiro, Rio Grande do Sul, Santa Catarina, São Paulo).

Tribe Dorynotini

Dorynota (s. str.) bidens (Fabricius, 1781)

Published records. Engenheiro Coelho (SIMões \& MonnÉ 2011); Rio Piracicaba (Simões \& SeKerka 2015).

Distribution. Brazil (Bahia, Espírito Santo, Minas Gerais, Pernambuco, Rio de Janeiro, São Paulo), French Guiana, Paraguay, and Trinidad and Tobago.

\section{Dorynota (s. str.) cornigera (Boheman, 1854)}

Published records. Arujá (BorowIEC \& TAKIZAWA 2011); Rosana (Porto Primavera); São Paulo (Simões \& SeKerka 2015).

Distribution. Argentina, Brazil (Distrito Federal, Goiás, Mato Grosso, Minas Gerais, Rio de Janeiro, Rio Grande do Sul, Santa Catarina, São Paulo), and Paraguay.

\section{Dorynota (s. str.) monoceros (Germar, 1823)}

Published records. Engenheiro Coelho (SIMõES \& MonNÉ 2011); Serra da Bocaína-Bananal; Peruíbe; São Paulo (Simões \& SeKerka 2015).

Distribution. Brazil (Bahia, Espírito Santo, Mato Grosso, Minas Gerais, Pará, Rio de Janeiro, Santa Catarina, São Paulo), Paraguay, Uruguay, and ? Venezuela.

\section{Dorynota (s. str.) parallela (Blanchard, 1842)}

Published records. Jundaí; Rio Claro; São Paulo; Vale do Anhangabaú (Simões 2014, Simões \& SeKerKa 2015).

Distribution. Argentina, Bolivia, Brazil (Bahia, Goiás, Mato Grosso, Minas Gerais, Pará, Rio de Janeiro, Santa Catarina, São Paulo), Peru, and Paraguay. 


\section{*Dorynota (s. str.) pugionata (Germar, 1823)}

Published records. São Paulo (Boheman 1857); Piracicaba (Toledo Piza 1968); São Paulo (Borowiec 1996); São José do Rio Preto (BoROWIEC 2002).

Additional record. BRAZIL: São PAulo: Serra de Bocaina, $1500 \mathrm{~m}$, xi.1965, 1 spec. (NHMB)

Distribution. Argentina, Bolivia, Brazil (Bahia, Espírito Santo, Goiás, Mato Grosso, Minas Gerais, Pará, Paraná, Rio Grande do Sul, Rio de Janeiro, Santa Catarina, São Paulo), and Paraguay.

\section{Paranota apiculata (Boheman, 1854)}

Published record. São Paulo (BoHEMan 1857).

Distribution. Brazil (Goiás, Mato Grosso, Pará, Rondônia, São Paulo).

\section{Paranota ensifera (Boheman, 1854)}

Published records. São Paulo (Boheman 1857, as P. gregaria (Boheman, 1854)); Amparo; 'Engenho Coelho [= Engenheiro Coelho]'; São Paulo (Simões 2014).

Distribution. Argentina, Bolivia, Brazil (Distrito Federal, Goiás, Mato Grosso, Mato Grosso do Sul, Minas Gerais, Rio de Janiero, São Paulo), Ecuador, and Paraguay.

\section{Paranota rugosa (Wagener, 1881)}

Published records. Indiana; São Paulo (SIMõEs 2014).

Distribution. Argentina, Bolivia, Brazil (Mato Grosso, São Paulo), and Paraguay.

\section{Paranota spinosa (Boheman, 1854)}

Published records. Mogi Guaçu (Buzzı 1988); Franca; Mogi GuaçuFazenda Campinha (SimõES 2014).

Distribution. Argentina, Bolivia, Brazil (Goiás, Mato Grosso, Mato Grosso do Sul, Minas Gerais, Pará, São Paulo), and Paraguay.

\section{Tribe Goniocheniini \\ *Chlamydocassis (s. str.) cribripennis (Boheman, 1850)}

Published records. Paiol Grande; Rio Pardo; São Bento do Sapucaí; São Paulo Capital; São Paulo-Ipiranga (VIANA 1964b); Parque Nacional do Itatiaia (BorowIEC 2002); Nonoai (Lopes et al. 2016).

Distribution. Argentina, Bolivia, Brazil (Bahia, Paraná, Rio Grande do Sul, Santa Catarina, São Paulo), and Paraguay.

\section{*Chlamydocassis (s. str.) metallica metallica}

(Klug, 1829)

Published record. Rio Pardo (VIANa 1964b).

Distribution. Argentina, Bolivia, Brazil (Mato Grosso do Sul, Pará, Rio de Janeiro, Rio Grande do Sul, São Paulo), Paraguay, and Uruguay.
Chlamydocassis (s. str.) perforata (Boheman, 1850)

Published record. São Paulo (BoHEMAN 1862).

Distribution. Argentina, Bolivia, ? Brazil (Rio Grande do Sul, São Paulo), and Peru.

Remarks. Presence of $C$. perforata should be verified by more material and examination of the published specimens. In general, species of Chlamydocassis Spaeth, 1952 in HINCKs (1952) are morphologically very variable and some populations are hard to identify correctly. Moreover, many species have quite a wide range through southern parts of tropical and subtropical South America, and local populations are distinct. The genus needs a complex revision, which should combine morphology with molecular methods to delimit the borders among species. Chlamydocassis perforata is the most common species of the genus in Bolivian savannas (Chiquitania, which is also its terra typica) and very likely reaches to the Brazilian state of Mato Grosso do Sul but based on the present material the species would not have a particularly wide range: VIANA (1964b) did not recognize the species in the material he studied despite having a very large sample of Argentinean Chlamydocassis, and the presence of $C$. perforata in Argentina is based on a single specimen from the province of Chaco (BOROWIEC 1996).

\section{Chlamydocassis (Ceratocassis) laticollis}

(Boheman, 1850)

Published records. Anhuagahg; Indiana; Rio Pardo (VIANA 1964b); Indiana (Buzzi 1988, as C. bicornuta (Boheman, 1850), misidentification).

Distribution. Argentina, Bolivia, Brazil (Mato Grosso, Pará, Rio Grande do Sul, São Paulo), Paraguay, and Venezuela.

Remarks. Bahia is removed from the range of $C$. laticollis as all records belong to C. bicornuta. See further remarks under the latter species in the section of Species excluded from the fauna of São Paulo.

\section{*Chlamydocassis (Ceratocassis) subcornuta (Boheman, 1850)}

Published record. Bocaina (BorowIEC 1996).

Distribution. Brazil (Minas Gerais, Rio de Janeiro, São Paulo).

\section{Goniochenia (s. str.) parvula Weise, 1896}

Published record. Bocaina (BorowIEC 1996).

Distribution. Brazil (Pernambuco, Rio de Janeiro, São Paulo).

Remarks. Goniochenia parvula was described from Cayenne in French Guiana (WeISE 1896); however, I find this peculiar as 1) additional specimens of G. parvula were collected in Eastern Brazil, and 2) all other species belonging to the nominotypical subgenus of Goniochenia Weise, 1896 are restricted to Eastern Brazil (from Pernambuco to Santa Catarina). Therefore, I assume that the material Weise had was mislabelled and thus the type locality of G. parvula is erroneous. French Guiana is hereby removed from the distribution of G. parvula. 


\section{Goniochenia (s. str.) quadraticollis (Boheman, 1850)}

Published records. Cantareira; Paranapiacaba; São Paulo (VIANA 1964b); 'Mendes [= collector; thus, São Paulo only]' (Borowiec 1996).

Distribution. Brazil (Espírito Santo, Minas Gerais, Rio de Janeiro, Santa Catarina, São Paulo).

Remarks. BorowieC (1996) published material labelled 'Sao Paulo Mendes' and interpreted Mendes as the name of locality. However, it is most likely a name of collector rather than locality (L. Borowiec, pers. comm. 2020) and thus, the record is here interpreted as São Paulo only.

\section{Zeugonota quadrinodosa (Boheman, 1857)}

Published records. São Paulo (BoHeman 1862, as Z. plicicollis (Boheman, 1862)); São Paulo (Spaeth 1914).

Distribution. Brazil (Goiás, Minas Gerais, Paraná, São Paulo).

Tribe Hemisphaerotini

Spaethiella speculicollis (Spaeth, 1928)

Published record. 'Borque da Saude [= Bosque da Saúde]' $($ SpaEtH 1928).

Distribution. Brazil (Pernambuco, Santa Catarina, São Paulo) and Paraguay.

\section{Spaethiella tristis (Boheman, 1850)}

Published record. Peruíbe (Borowiec 1996).

Distribution. Brazil (Bahia, Minas Gerais, Pará, Santa Catarina, São Paulo) and Ecuador.

Tribe Imatidiini

\section{*Calliaspis brevicornis Spaeth, 1905}

Published records. São Paulo (SPAETh 1905); Jabaguara (BorowIEC 1996).

Distribution. Brazil (Paraná and São Paulo).

\section{Calliaspis cinnabarina Boheman, 1850}

Published record. Amparo (SIMões \& MonNé 2011).

Distribution. Brazil (Bahia, ? Rio de Janeiro, ? São Paulo), French Guiana, Peru, and Suriname.

Remarks. SimõES \& MonNÉ (2011) recorded this species from Rio de Janeiro (P.N. Itatiaia) and São Paulo for the first time. They also provided a colour photograph of one specimen in dorsal view, but it is not known which population they used. The voucher specimens from São Paulo were deposited in MNRJ and destroyed by the fire in 2018. Unfortunately, the photograph is of a rather low resolution and does not allow recognition of the species with certainty.

Calliaspis cinnabarina was described from 'Cayenna, Brasilia' and is certainly distributed over the Guiana shield and the adjacent Amazon. However, another species, C. umbonata, was described from southern Brazil (Santa Catarina), differing from $C$. cinnabarina in conspicuous postscutellar tubercle with concave posterior slope in la- teral view while $C$. cinnabarina has only slightly irregular elytra in the postscutellar point. Therefore, the specimens recorded by SimõES \& MonNÉ (2011) could belong to either of the two species. Judging from the photograph the beetle looks more similar to $C$. umbonata as it has uniformly yellow and longer antennae (usually at least with darkened tips in C. cinnabarina) and the reflection in the postscutellar area gives the impression of a higher tubercle but it is merely my feeling rather than reasonable discrimination. Thus, I leave both records within the distribution of C. cinnabarina but particularly that from São Paulo must be verified by additional material as $C$. umbonata is here recorded as new to São Paulo. Calliaspis cinnabarina has been recently recorded also from Bahia (ALBERTONI 2017) based on specimens I compared with the type material that agreed fairly well.

\section{*Calliapis umbonata Hincks, 1956}

New record. BRAZIL: São PAulo: without further data, 1 spec., J. Mráz leg. (NMPC).

Distribution. Brazil (Santa Catarina). New to São Paulo.

\section{Cephaloleia abdita Staines, 2014}

Published record. Cerqueira Cesar (Staines \& García-Robledo 2014).

Distribution. Brazil (São Paulo).

*Cephaloleia caeruleata Baly, 1875

$$
\text { (Figs 8-10) }
$$

Cephaloleia caeruleata Baly, 1875: 75. Type locality: Brazil, Rio de Janeiro, Nova Friburgo.

Cephaloleia dilatata Uhmann, 1948a: 220. Type locality: Brazil, Minas Gerais, Virginia-Fazenda Campos.; syn. nov.

Type material examined. Cephaloleia caeruleata: HoLOTyPe (Fig. 8): + , pinned, 'Type $\mid$ H. T. [w, p, cb, circle label with red frame] $\|$ N. Frib. [green, hw, cb] || caeruleata [w, hw by Baly cb] || Baly Coll. [w, p, cb] || Cephaloleia | caeruleata $\mid$ Baly $\mid \mathrm{N}$ : Friburg [green, hw, cb]' (BMNH).

Cephaloleia dilatata: HOLOTYPE: ${ }^{2}$, pinned (missing right antenna), 'Virginia - 1500 m | S. Minas-Geraes | Faz. Campos | v. - 1919 [hw] | J.F.Zikán [w, p, cb] || No. [p] 28 [hw] | J.F.Zikán [w, p, s, bf] || Cephaloleia $\widehat{O}[\mathrm{hw}]$ | dilatate uh [hw] | UHMANN DET. 4[p]5[hw] [w, p + hw by Uhmann, s] || Holotypus [r, hw by Uhmann, s] || Kopf u. Hlsch. | 1945 [brown, hw by Uhmann, cb] || Oberseite | 1946 [brown, hw by Uhmann, cb] || DEI Müncheberg | Col - 02785 [green, p, s]' (SDEI).

Additional material examined. BRAZIL: São PAULO: without further data, 4 ô 3 우, J. Mráz leg. (NMPC, 2 LSPC).

Distribution. Brazil (Minas Gerais, Rio de Janeiro). New to São Paulo.

Remarks. BALY (1875) based his description explicitly on a single specimen but did not compare the species to any other. UHMANN (1948a) also based his description on a single specimen and compared it to three dark brown coloured species with depressed elytra but stated that $C$. dilatata is distinct by the shape of pronotum and metallic green colour of the dorsal side. STAINES \& GARCÍA-ROBLEDo (2014) considered both taxa valid and separated $C$. caeruleata by antennomere I shorter than III (vs. longer in C. dilatata), elytral punctures larger laterally (vs. smaller) and prosternum rugose laterally (vs. alutaceous). The figure (fig. 92) they published for C. caeruleata is not in 


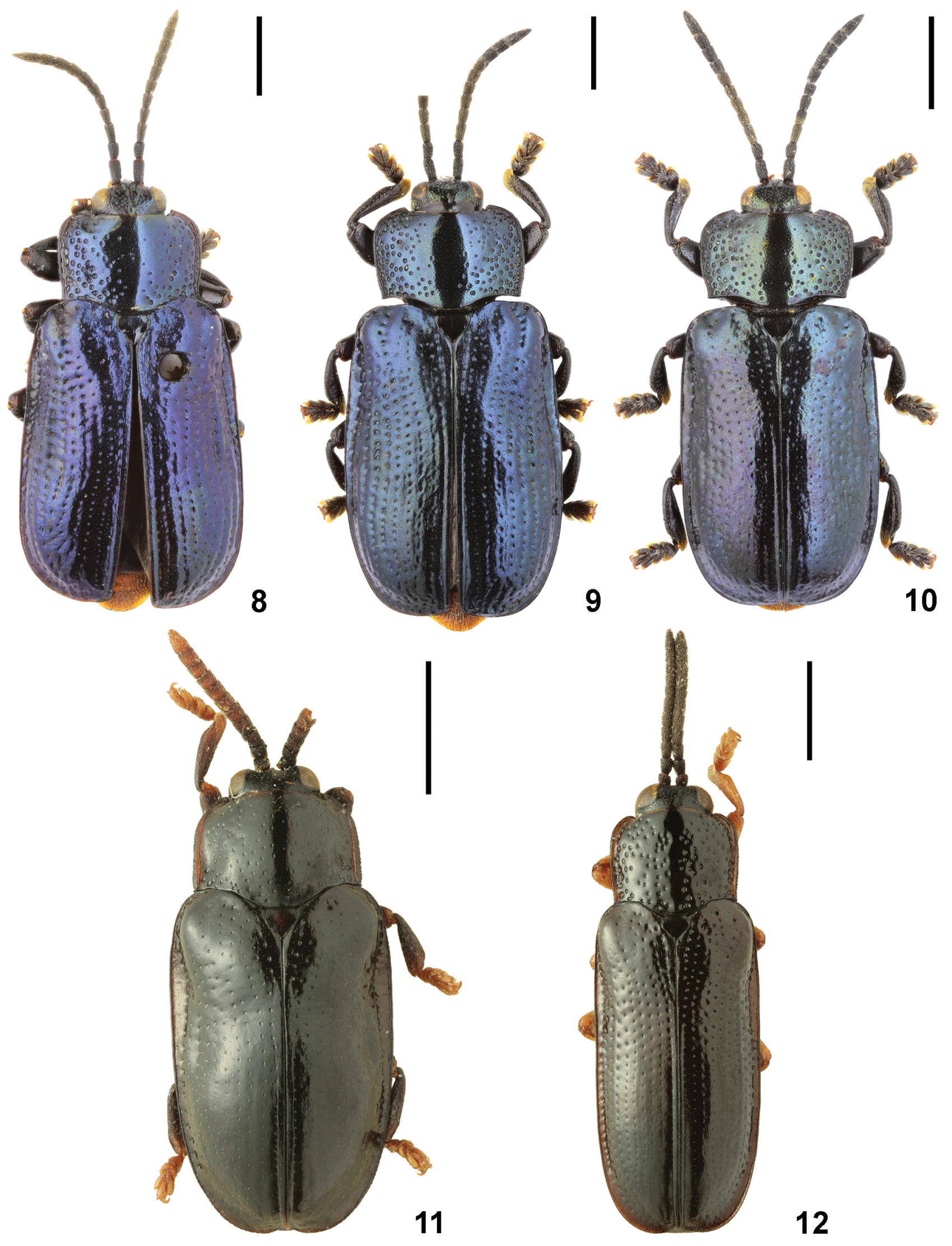

Figs 8-12. Dorsal aspects. 8-10 - Cephaloleia caeruleata Baly, 1875: 8 - holotype, $9 ; 9$ - 9 collected by J. Mráz; 10 - đallected by Mráz and compared to the holotype of C. dilatata Uhmann, 1948; 11 - Cephaloleia impressa Uhmann, 1930, holotype; 12 - Cephaloleia nitida Uhmann, 1930, paratype. Scale bars $=1 \mathrm{~mm}$. 
agreement with the holotype and belongs to a completely different species; the figure of $C$. dilatata was taken from the holotype specimen.

I have studied holotypes of both taxa and compared them to the series of specimens collected by J. Mráz and they belong to a single species. I could not see the differences in lengths of antennomeres which are in all specimens proportionally the same. Also, the structure of hypomera is very similar in all specimens. On the other hand, the difference in the coarseness of the punctation on elytra is obvious and it is a result of sexual dimorphism. Males (Fig. 10) have strongly shiny (polished) elytra, which are completely shiny and finely punctate; their lateral slopes are slightly irregular and have slightly coarser punctation than in the sutural rows but in general appearance, elytra are smooth, while females (Figs 8-9) have much coarser punctation on the lateral slope, the area is conspicuously irregular and velvet-like, not shiny. The entire series collected by J. Mráz has dorsum obscurely turquoise green; the colouration is constant and male specimens (Fig. 10) perfectly match the holotype of $C$. dilatata also structurally. Holotype of $C$. caeruleata is dark blue with distinctly coarser structure of elytra than in female specimens collected by Mráz but the remaining characters are the same. Therefore, I hereby synonymize C. dilatata with $C$. caeruleata.

Staines \& García-Robledo (2014) published three additional specimens for $C$. caeruleata, however, four collecting dates were listed. One specimen was from Rio de Janeiro; two from Santa Catarina: Nova Teutonia, and the last one from Ecuador: San Gabriel. I am suspicious with regard to the correctness of this identification as the photograph they presented does not match C. caeruleata and it was taken either for the specimen from Rio de Janeiro or Ecuador. Moreover, the record from Ecuador is very improbable simply for the biogeographical reasons and thus is not adopted here. Based on the photograph provided by Ed Riley the two specimens from Santa Catarina belong to Cephaloleia impressa Uhmann, 1930, not C. caeruleata. The species occurs with certainty in Rio de Janeiro as the holotype originates from there; however, I did not have the opportunity to study the specimen published by Staines \& García-Robledo (2014).

\section{Cephaloleia deyrollei Baly, 1859}

Published records. São Paulo (WeISE 1910a); 'Vila Darvin [field station of MZUSP at that time]' (LÜDERWALDT 1910); Santos; Estação Biológica de Paranapiacaba (Uhmann 1964); Casa Grande (Staines \& GARCÍA-Robledo 2014).

Distribution. Brazil (Bahia, Paraná, Rio de Janiero, Santa Catarina, São Paulo).

Remarks. StAINES \& GARcíA-Robledo (2014) recorded this species from Bolivia, Ecuador and French Guiana for the first time; however, these records are dubious and most likely represent misidentifications. Species of Cephaloleia Chevrolat, 1836 are somewhat restrictive considering their distribution and habitat preferences, with only several species truly widespread. The same is true for their host plants, in this case Calathea and/or He- liconia species. Therefore, the abovementioned countries are removed from the distribution of $C$. deyrollei, which is restricted to eastern Brazil. However, the respective specimens should be studied to confirm this action.

\section{Cephaloleia elaeidis Maulik, 1924}

Published record. Cantareira (Staines \& García-Robledo 2014).

Distribution. Brazil (Bahia, São Paulo) and ? Ecuador. Remarks. The records from Ecuador (STAINES \& GARCÍA-Robledo 2014) are dubious and the species was probably misidentified. On the other hand, the species is a pest on palms and thus could have been imported to Ecuador. Also, the record from São Paulo should be verified to confirm its identity as many species feeding on palm trees are morphologically similar.

\section{* Cephaloleia flavovittata Baly, 1859}

New record. BRAZIL: São PaUlo: without further data, 8 spec., J. Mráz leg. (NMPC, 1 LSPC).

Distribution. Brazil (Bahia). New to São Paulo.

Remarks. The species was described generally from Brazil (BALY 1859) and subsequently listed only in catalogues (e.g., UHMANn 1957). The only record so far was published by BONDAR (1938) from Bahia but it was not mentioned in species distribution in the recent revision (Staines \& García-Robledo 2014). The species is here newly recorded from São Paulo and very likely occurs in all east Brazilian states.

\section{Cephaloleia fulvipes Baly, 1859}

Published record. Cantareria (Uhmann 1964, Staines \& García -RobLedo 2014).

Distribution. Brazil (São Paulo).

Remarks. UHMANN (1964) published two specimens collected in Cantareira on 29 October 1939; STAINES \& GARCÍA-Robledo (2014) published the same specimens again and added some additional ones collected on different dates. The occurrence in Ecuador (STAINES \& GARCÍA-ROBLEDo 2014) is dubious and probably based on misidentification, and thus it is removed from the species range.

\section{* Cephaloleia impressa Uhmann, 1930 (Fig. 11)}

Published record. São Paulo (Uhmann 1930).

Type material examined. НоLотуре (Fig. 11): glued, missing right antenna from antennomere VI on, 'SAÕ PAULO | BRAS.MRÁZ LGT. | MUS.PRAGENSE [w, p, cb, bf] || HOLOTYPE [r, t, s] || Cephaloleia | impressa n. sp. | det. Uhmann 28. [w, hw by Uhmann, s] || Collect. | Spaeth [w, p, cb]' (NHMW).

Additional material examined. BRAZIL: São PAULO: without further data, 9 spec., J. Mráz leg. (NMPC, 3 LSPC).

Distribution. Brazil (Santa Catarina, São Paulo).

Remarks. Spaeth also identified part of the hispines and retained duplicates for his collection. Among them were also specimens which he was not able to identify to species, so he sent them later to Uhmann but probably without mentioning that more specimens exist in NMPC 
collection. As a result, $C$. impressa was described based on the holotype assigned to Spaeth's collection, even though there were more specimens in NMPC.

Staines \& García-Robledo (2014) published two specimens from Nova Teutonia (Santa Cataria) as $C$. caerulescens; however, based on the photograph provided by Ed Riley they were misidentified and belong to C. impressa.

\section{Cephaloleia linkei Uhmann, 1939}

Published record. Campos do Jordão (Uhmann 1964).

Distribution. Brazil (Santa Catarina, São Paulo).

\section{Cephaloleia marantae Uhmann, 1957}

Published record. São José dos Campos (Staines \& García-Robledo 2014).

Distribution. Argentina, Bolivia, Brazil (Paraná, Rio Grande do Sul, Santa Catarina, São Paulo), and Paraguay. Remarks. Staines \& García-Robledo (2014) published material from 'Porto Alegre, 17 October 1951' deposited in USNM and scored it for the state of Amazonas. I have not seen the respective specimen(s), but it must certainly be a misinterpretation of the locality data as $C$. marantae is distributed in the Paraná Basin. Most likely the locality refers to Porto Alegre in Rio Grande do Sul, and this would be supported also by the year 1951 as F. Monrós (collection nowadays in USNM) received numerous specimens from this period from P. Buck, who was based in Porto Alegre, RGS.

\section{*Cephaloleia nitida Uhmann, 1930 (Fig. 12)}

Published records. São Paulo (Uhmann 1930, Staines \& García-RobleDo 2014); Cubatão (Uhmann 1964).

Type material examined. HоLOTYPE: glued, missing terminal antennomeres in both antennae 'SAÕ PAULO | BRAS.MRÁZ LGT. | MUS. PRAGENSE [w, p, cb, bb] \|| HOLOTYP [r, t, s] || PARATYPE [r, hw by Uhmann, s] || Cephaloleia [hw] | nitida Uh. [hw] | UHMANN DET. [p] 29 [hw] [w, p + hw by Uhmann, s]' (SDEI); PARATYPE (Fig. 12): glued, 'SAÕ PAULO | BRAS.MRÁZ LGT. | MUS.PRAGENSE [w, p, cb, bb] || C. nitida | Uhm. cotyp. [w, hw by Spaeth, cb] || CO TYPUS [pink, p, cb] || Collect. | Spaeth [w, p, cb]' (NHMW).

Additional material examined. BRAZIL: São PAULO: without further data, 10 spec., J. Mráz leg. (NMPC, 2 LSPC).

Distribution. Argentina and Brazil (Santa Catarina, São Paulo).

Remarks. Spaeth was unable to identify this species and retained three specimens for his collection. Later on, he sent at least one to Uhmann for identification but without mentioning existence of other specimens in NMPC. UHMANN (1930) described the species as $C$. nitida explicitly based on two specimens and stated that the holotype was in his collection (now SDEI) and the paratype in Spaeth's collection (hispines now in NHMW). However, I assume that Uhmann saw only the holotype as the paratype specimen does not bear his identification labels, while he usually labelled all type specimens he had examined. Moreover, there is a third specimen in Spaeth's collection, which Spaeth provided with his pink cotype label, but it does not belong to the type series as the species was based on two specimens only. In NMPC, there is a series of specimens belonging to this species, but they were not included in the type series and until now remained identified only as Cephaloleia sp. by Spaeth.

\section{Cephaloleia picta Baly, 1859}

Published record. Cantareira (Uhmann 1960).

Distribution. Argentina, Brazil (Bahia, Rio de Janeiro, Santa Catarina, São Paulo), and Paraguay.

\section{*Cephaloleia teutonica Uhmann, 1937}

Published records. Rio Grande-Repreza near São Paulo Capital; Salesópolis-Estação Biológica de Boraceia (Uhmann 1964); Cantareira; São Paulo; São Paulo-Botanical Garden (StaInes \& GarcíA-Robledo 2014). Additional record. São Paulo: Ribeirão Pires, xi.1898, 1 spec., E. Gounelle leg. (MNHN).

Distribution. Brazil (Rio Grande do Sul, Santa Catarina, São Paulo).

\section{*Cephaloleia trilineata Uhmann, 1942}

New record. São Paulo: without further data, 1 spec., J. Mráz leg. (NMPC).

Distribution. Brazil (Rio de Janeiro). New to Minas Gerais and São Paulo.

Remarks. StAines \& GARCÍA-Robledo (2014) published a new record for this species as follows: 'BRAZIL: São Paulo- Río Piracicaba, February 1885 (USNM). Total: 1.' In Brazil, there are at least two Piracicaba Rivers, one in Minas Gerais and the other in São Paulo. Although they did not provide further data, I am quite sure that the specimen was collected by Philibert Germain as I have examined various specimens of Cassidinae with this locality data in MNHN. The exact transcription of the locality label is: 'Brésil Rio Piracicaba P. Germain Février 1885'. According to Horn \& KAHLE (1935), Germain collected in Brazil in the state of Minas Gerais. Consequently, the record of $C$. trilineata is here transferred and the species is recorded as new to Minas Gerais. However, the species is here recorded also for São Paulo as J. Mráz collected one specimen. It differs from the holotype in absence of lateral black stripes, which are only obscurely indicated near humeri; also, the sutural stripe vanishes towards the midlength. Otherwise, the specimen seems to match the holotype fairly well.

\section{Cephaloleia tucumana Weise, 1904}

Published record. São Paulo Capital-Rua Maestro Cardim (UhmanN 1964).

Distribution. Argentina and Brazil (Bahia, São Paulo).

\section{*Cephaloleia vittipennis Weise, 1910}

Published records. Santos (WeIse 1910a); Cubatão; Estação Biologica de Paranapiacaba (Uhmann 1964); Cantareira; Santos (Staines \& GarCÍA-RoBLEDo 2014).

Distribution. ? Argentina and Brazil (Santa Catarina, São Paulo). 
Remarks. Staines \& GARcíA-RoBledo (2014) recorded this species from Argentina, Peru and Venezuela for the first time. Occurrence in Argentina is possible; however, the record must be taken with caution as the revision contains numerous misidentifications and misinterpretations. Occurrence in Peru and Venezuela is very improbable as the entire $C$. deyrollei species-group is restricted to eastern Brazil, and the two countries are removed from the species distribution.

\section{*Cephaloleia zikani Uhmann, 1935}

New record. BRAZIL: São Paulo: without further data, 3 spec., J. Mráz leg. (NMPC, 1 LSPC).

Distribution. Brazil (Rio de Janeiro, Santa Catarina). New to São Paulo.

Remarks. One specimen matches the holotype fairly well. The other has somewhat narrower pronotum but the other structures seem to be the same, and I consider it to be intraspecific variability.

\section{*Stenispa vespertina Baly, 1877}

New record. BRAZIL: São Paulo: without further data, 1 spec., J. Mráz leg. (NMPC).

Distribution. Brazil (Bahia, Rio de Janeiro, Santa Catarina). New to São Paulo.

\section{*Stenispa viridis (Pic, 1931)}

New record. BRAZIL: São PAulo: without further data, 5 spec., J. Mráz leg. (NMPC, 2 LSPC).

Distribution. Brazil (Minas Gerais). New to São Paulo. Remarks. Until now, this species was known only from the type specimen in MNHN described from 'Minas' and recently transferred from the genus Cephaloleia to Stenispa Baly, 1859 (SEKERKA 2014).

\section{*Xenispa bicolorata (Uhmann, 1948)}

New records. BRAZIL: São Paulo: without further data, 1 spec., J. Mráz leg. (NMPC); Vale do Rio Prado, xii.1898, 5 spec., E. Gounelle leg. (MNHN, 2 LSPC).

Distribution. Brazil (Santa Catarina) and Paraguay. New to São Paulo.

\section{Xenispa grayella (Baly, 1859)}

Published records. Alto da Serra; Salesópolis-Estação Biológica de Boraceia (Uhmann 1964).

Distribution. Brazil (Paraná, Rio de Janeiro, São Paulo).

\section{Xenispa plaumanni (Uhmann, 1937)}

Published record. Campos do Jordão (Uhmann 1964).

Distribution. Brazil (Rio Grande do Sul, Santa Catarina, São Paulo) and Paraguay.

\section{Xenispa pygidialis (Uhmann, 1940)}

Published record. Salesópolis-Estação Biológica de Boraceia (UHMANN 1964).
Distribution. Brazil (Rio Grande do Sul, Santa Catarina, São Paulo) and Paraguay.

Tribe Ischyrosonychini

\section{Cistudinella apiata (Boheman, 1854)}

Published record. São Paulo (SPAETh 1923).

Distribution. Brazil(Rio de Janeiro, Santa Catarina, São Paulo).

*Cistudinella notata (Boheman, 1854)

Published record. Butanta (BorowIEC 1996).

Distribution. Argentina, Brazil (Bahia, Minas Gerais, Paraná, Rio de Janeiro, Rio Grande do Sul, Santa Catarina, São Paulo), and Paraguay.

\section{Cistudinella obducta (Boheman, 1854)}

Published record. Nonoai (LoPEs et al. 2016).

Distribution. Argentina, Bolivia, Brazil (Distrito Federal, Mato Grosso, Mato Grosso do Sul, Minas Gerais, Pará, Paraná, Rio Grande do Sul, Rondônia, Santa Catarina), Paraguay, Peru, and Venezuela.

\section{Cistudinella rufitarsis Spaeth, 1905}

Published record. 'Mendes [= collector; thus only São Paulo]' (BoROwIEC 1996).

Distribution. Brazil (São Paulo).

Remarks. BorowiEc (1996) published material labelled 'Sao Paulo Mendes' and interpreted Mendes as the name of locality. However, it is most likely a name of collector rather than locality (L. Borowiec, pers. comm. 2020) and thus, the record is here interpreted only as São Paulo.

\section{Eurypedus peltoides (Boheman, 1854)}

Published record. São Paulo (BoHEMAN 1854).

Distribution. Argentina, Bolivia, Brazil (Bahia, Espírito Santo, Minas Gerais, Paraná, Rio de Janeiro, Rio Grande do Sul, São Paulo), and Paraguay.

\section{*Orexita postica (Boheman, 1855)}

Published record. Cantareira; Sampaio (Borowiec 1996, as O. picta (Boheman, 1855)).

Distribution. Argentina, Brazil (Minas Gerais, Rio de Janeiro, Rio Grande do Sul, Santa Catarina, São Paulo), and Paraguay.

Remarks. Until recently, this taxon was reported as $O$. picta; however, the name $O$. postica has the priority (SEKERKA 2016).

Mesomphaliini

\section{*Acromis spinifex (Linnaeus, 1763)}

Published records. Indiana; Mogi das Cruzes; Santos; São Vicente (BuZzI 1988, also as A. nebulosa (Boheman, 1854)); Barueri; Cantareira; Guaruja near Santos (Borowiec 1996); Bertioga; Campinas; Cantareira; Campos do Jordão; Ilha Porchat; Mogi das Cruzes (as 'M. da Cruses' and perhaps also 'Loggdasbruzes'); Santos; São Paulo; São Vicente (Снавоо 2001); 
Peruíbe-Estação Ecológica da Juréia-Itatins (BorowiEc 2002). Additional records. BRAZIL: São Paulo: Santos, v.1883, 1 2 우

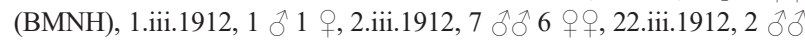
2 우, G. E. Bryant leg. (BMNH).

Distribution. Argentina, Bolivia, Brazil (Bahia, Espírito Santo, Goiás, Mato Grosso, Mato Grosso do Sul, Minas Gerais, Pará, Paraná, Rio de Janeiro, Santa Catarina, São Paulo), Colombia, Ecuador, French Guiana, Guyana, Paraguay, Peru, Suriname, Trinidad and Tobago, and Venezuela.

\section{Agenysa caedemadens (Lichtenstein, 1796)}

Published records. 'Copla do Alto [= Capela do Alto]'; 'Sao Paulo Sitio [= ? São Paulo Capital]' (Borowiec 1996, as A. guianiensis (Boheman, 1857)).

Distribution. Argentina, Brazil (Amazonas, Mato Grosso, Pará, ? São Paulo), Curaçao, French Guiana, Suriname, and Venezuela.

Remarks. Agenysa guianiensis was recently synonymized with $A$. caedemadens as it is only a uniformly metallic-coloured form of the same species. It is distributed primarily in the Guiana Shield and the Lower Amazon Basin, and records from the interior of South America were rejected (SEKERKA 2016). The species was recorded from São Paulo only once based on seven specimens collected by E. Rivier in 1976 (BorowIEC 1996). Indeed, they could have been mislabelled but probably not as in the same paper, there are published many specimens of other species collected by E. Rivier in São Paulo, and all are reasonable records with the exception of A. guianiensis. Unfortunately, I have not had the opportunity to examine these specimens yet, thus the occurrence of $A$. caedemadens should be considered dubious until more material is found.

\section{Agenysa parellina (Boheman, 1850)}

Published record. Sorocaba (VIANA 1968).

Distribution. Argentina, Brazil (Mato Grosso, Minas Gerais, Rio Grande do Sul, Santa Catarina, São Paulo), Paraguay, and Uruguay.

\section{Amythra valida (Boheman, 1855)}

Published record. Campinas (BorowIEC 1996).

Distribution. Argentina and Brazil (São Paulo).

\section{*Anacassis candida (Boheman, 1854)}

New record. BRAZIL: São Paulo: without further data, 1 spec., J. Mráz leg. (NMPC).

Distribution. Brazil (Minas Gerais). New to São Paulo.

\section{*Anacassis cribrum (Klug, 1829)}

Published record. Casa Grande (Buzzi 1988).

Distribution. Argentina, Brazil (Paraná, Rio Grande do Sul, Santa Catarina, São Paulo), and Uruguay.

\section{*Anacassis dubia (Boheman, 1854)}

Published records. Butantan; Cantareira; São Paulo (Borowiec 1996).
Distribution. Argentina and Brazil (Minas Gerais, Paraná, Rio de Janeiro, Rio Grande do Sul, Santa Catarina, São Paulo).

\section{*Anacassis fuscata (Klug, 1829)}

Published records. Paranapiacaba (Buzzi 1988); Barueri; Cantareira; São Paulo (Borowiec 1996); São Roque-Sitio Itatuba (Borowiec 2002); Campinas (BorowIEC 2009).

Additional records. BRAZIL: São Paulo: Amparo, 4.-30.iv.1982, 1 spec., Wewalka leg. (NHMW); Cantareira, 10.vii.1923, 1 spec., G. L. R. Hancock leg. (BMNH); Itararé, xii.1935, 1 spec., D. Braz leg. (NHMB); Santos, 5.iv.1912, 1 spec., G. E. Bryant leg. (BMNH); São Paulo Capital, v.1883, 4 spec., A. Fry leg. (BMNH), 20.ii.1912, 1 spec., G. E. Bryant leg. (BMNH).

Distribution. Argentina, Bolivia, Brazil (Minas Gerais, Paraná, Rio de Janeiro, Rio Grande do Sul, Santa Catarina, São Paulo), Paraguay, and Uruguay.

\section{*Anacassis phaeopoda Buzzi, 1976}

Published records. Campos do Jordão (BorowIEC 1996); Serra do Japi (NogueIRA-DE-SÁ \& VASCONCELlos-Neto 2003a,b).

Additional records. BRAZIL: São Paulo: São Paulo Capital, 19.ii.1912, 1 spec., 28.ii.1912, 1 spec., G. E. Bryant leg. (BMNH).

Distribution. Brazil (Paraná, Rio Grande do Sul, Santa Catarina, São Paulo).

\section{Anacassis punctulata (Klug, 1829)}

Published record. Campos do Jordão (BoROwIEC 1996).

Distribution. Brazil (Minas Gerais, Paraná, Rio de Janeiro, Rio Grande do Sul, Santa Catarina, São Paulo).

Anacassis rubroornata (Boheman, 1854)

Published record. Bocaina (BOROWIEC 1996).

Distribution. Brazil (São Paulo).

\section{Anacassis testudinaria (Boheman, 1850)}

Published record. Cantareira-Parque do Estado de São Paulo (SIMõES \& MONNÉ 2008).

Distribution. Argentina and Brazil (Espírito Santo, Minas Gerais, Paraná, Rio de Janeiro, Rio Grande do Sul, Santa Catarina, São Paulo).

\section{Botanochara haematodes (Perty, 1832)}

Published record. Nova Granada (BorowIEC 1996).

Distribution. Brazil (Bahia, Mato Grosso, São Paulo) and Peru.

\section{*Botanochara impressa (Panzer, 1798)}

Published records. São Paulo (Haitlinger 1991, as B. texta (Boheman, 1850)); Piracicaba (Toledo Piza 1968, as B. nervosa (Fabricius, 1801)); Sumaré (Costa et al. 1988); Monte Azul (BorowieC 1996); São RoqueSitio Itatuba (BoRowiEC 2002).

Distribution. Argentina, Bolivia, Brazil (Amapá, Amazonas, Goiás, Maranhão, Minas Gerais, Pará, Paraná, Rio de Janeiro, Santa Catarina, São Paulo, Tocantins), Paraguay, and Peru. 
Botanochara impressipennis (Boheman, 1850)

Published record. Ilha Seca (SouZa Lopes 1940). Additional record. BRAZIL: São PAulo: Amparo, 4.-30.iv.1982, 2 spec., Wewalka leg. (NHMW, 1 LSPC).

Distribution. Brazil (Goiás, Mato Grosso do Sul, Mato Grosso, São Paulo).

\section{Botanochara nigropicta (Boheman, 1850)}

Published record. Engenheiro Coelho (SIMÕES \& MonNÉ 2008).

Distribution. Brazil (Goiás, Mato Grosso, Minas Gerais, São Paulo) and Paraguay.

\section{Botanochara quinquefasciata (Perty, 1832)}

Published record. Angatuba (SIMÕES \& MonNÉ 2008).

Distribution. Brazil (Mato Grosso, Paraná, Rio Grande do Sul, São Paulo).

\section{Botanochara rubroguttata (Boheman, 1850)}

Published records. São Paulo (BoHEMAn 1857, as B. anthracina (Boheman, 1857); SPAETH 1940).

Distribution. Argentina, Brazil (Goiás, Minas Gerais, São Paulo), and Paraguay.

\section{Botanochara ruforeticulata (Boheman, 1850)}

Published record. São Paulo (SPAETH 1940).

Additional record. BRAZIL: SÃo PAulo: Serra de Bocaina, 1500 m, xi.1965, 1 spec. (NHMB).

Distribution. Brazil (Bahia, Espírito Santo, Mato Grosso, Minas Gerais, Rio de Janeiro, São Paulo).

\section{Botanochara sedecimpustulata (Fabricius, 1781)}

Published record. São Paulo (Borowiec 1996).

Distribution. Argentina, Bolivia, Brazil (Amazonas, Minas Gerais, Pará, Piauí, Rondônia, São Paulo), Paraguay, and Uruguay.

\section{Botanochara sigillata (Spaeth, 1940)}

Published record. São Paulo (SPAETh 1940).

Distribution. Brazil (São Paulo).

\section{Botanochara tesselata (Burmeister, 1870)}

Published record. Saltinho (LOPES et al. 2016).

Distribution. Argentina, Bolivia, Brazil (Ceará, Goiás, Mato Grosso do Sul, Pará, Rio de Janeiro), and Paraguay.

\section{Chelymorpha commutabilis Boheman, 1854}

New record. BRAZIL: São PAULO: without further data, $1 \mathrm{spec}$. (BMNH). Distribution. Brazil (Minas Gerais). New to São Paulo.

*Chelymorpha constellata (Klug, 1829)

New records. BRAZIL: São PAulo: without further data, 1 , J. Mráz leg. (NMPC); Guarujá-Perequê, 23.x.1994, 1 spec., W. Wittmer leg.
(NHMB); Santos, 22.xi.1911, 3 spec., 22.ii.1912, 7 spec., 2.iii.1912, 4 spec., 18.iii.1912, 2 spec., 17.iv.1912, 1 spec., G. E. Bryant leg. (BMNH).

Distribution. Brazil (Distrito Federal, Minas Gerais, Pará, Rio de Janeiro, Rio Grande do Sul, Santa Catarina), French Guiana, and Peru. New to São Paulo.

\section{*Chelymorpha inflata Boheman, 1854}

Published records. São Paulo-Ipiranga (as C. infaceta Boheman, 1854); Butanta; Cantareira (BorowIEC 1996); São Roque-Sitio Itatuba (BOROWIEC 2002).

Distribution. Argentina, Brazil (Pará, Paraná, Rio de Janeiro, Rio Grande do Sul, Santa Catarina, São Paulo), Ecuador, and Paraguay.

\section{Chelymorpha limbatipennis Spaeth, 1926}

Published record. São Paulo (SPAETh 1926c).

Distribution. Brazil (Rio Grande do Sul, São Paulo).

\section{*Chelymorpha multipunctata (Olivier, 1790)}

Published records. Campinas (VASConcellos-Neto 1988, as C. cribraria auct.); Guarulhos (BuzZI 1988, as C. rufipennis Boheman, 1854); Bocaina (Borowiec 1996, as 'rufipennis' form); São Paulo (BorowieC 2002, as C. cribraria auct.); Jundiaí (BorowieC \& TAKIZAWA 2011, as C. cribraria auct.); Rio Claro; Saltinho (Lopes et al. 2016, as C. cribraria auct.).

Additional record. BRAZIL: São Paulo: Taubaté, 27.x.2005, $1 \mathrm{spec}$., Z. Tüdös leg. (OKZC).

Distribution. Argentina, Bolivia, Brazil (Amapá, Amazonas, Distrito Federal, Espírito Santo, Goiás, Mato Grosso, Minas Gerais, Pará, Paraná, Rio de Janeiro, Rio Grande do Norte, Rio Grande do Sul, São Paulo), Colombia, Costa Rica, Dominica, Dominican Republic, Ecuador, French Guiana, Grenada, Guadeloupe, Panama, Paraguay, Peru, St. Vincent, Trinidad, USA, and Venezuela.

Remarks. For a long time, this taxon was known under the name $C$. cribraria; however, the name was misapplied and true $C$. cribraria was synonymized with $C$. cassidea (Fabricius, 1775). Thus, the oldest available synonym became the valid name for this taxon (SEKERKA \& BARCLAY 2014).

\section{*Chelymorpha nigricollis Boheman, 1854}

Published record. Campos do Jordão (BorowIEC 1996).

Distribution. Bolivia, Brazil (Amazonas, Bahia, Paraná, Rio Grande do Sul, Santa Catarina, São Paulo), Paraguay, and Peru.

\section{Chelymorpha reimoseri Spaeth, 1928}

Published records. Campinas (BOROwIEC 2009, BotTCHER et al. 2009).

Distribution. Argentina and Brazil (São Paulo).

\section{Chelymorpha subpunctata Boheman, 1854}

Published record. São Paulo (Borowiec \& SKUZA 2004).

Distribution. Brazil (São Paulo). 


\section{*Cyrtonota cyanea (Linnaeus, 1758)}

Published record. Santos (Hincks 1956).

Additional records. BRAZIL: São Paulo: without further data, 1 , J. Mráz leg. (NMPC); Alto da Serra, 9.iii.1912, 5 spec., G. E. Bryant leg. (BMNH); Guarujá-Perequê, 23.x.1994, 1 spec., W. Wittmer leg. (NHMB); Santos, 22.xi.1911, 3 spec., 23.iii.1912, 1 spec., 4.iv.1912, 3 spec., G. E. Bryant leg. (BMNH).

Distribution. Brazil (Rio de Janeiro, Santa Catarina, São Paulo).

Remarks. HincKs (1956) published a specimen labelled only 'Santos' and wrote the following: '[this specimen] agrees closely with a specimen from the same locality in the Spaeth collection and is exceptional in being of small size and entirely black in colour.' I am quite sure that Santos refers to the well-known municipality in São Paulo as well as that the specimen in Spaeth's collection belongs to $C$. cyanea, thus this note constitutes the first published record for the state. However, I am not sure about the identity of the small and black specimen mentioned by Hincks. Black colouration in C. cyanea is quite common and is either natural or caused post mortem by the wax from the body (it can be cleaned by some dissolvent). But the small size is very unusual as one of the principal characters of $C$. cyanea is large body size, being distinctly larger than the other species of this group. I did not have the opportunity to examine the specimen, but my feeling is that it belongs rather to $C$. thalassina (Boheman, 1850), characterized by smaller size and also is more abundant in São Paulo than C. cyanea.

In the same publication Hincks also recorded one specimen from Peru (San Pedro, ii.[19]38, F. Tippmann). Cyrtonota cyanea is restricted to the eastern coast of Brazil and the record from Peru is very improbable. It is possible that the specimen was imported with some fruits or was mislabelled or misidentified, and thus the species is removed from the fauna of Peru.

\section{Cyrtonota sexpustulata (Fabricius, 1781)}

Published record. Bocaina (BorowIEC 1996).

Distribution. Brazil (Distrito Federal, Minas Gerais, Paraná, Rio de Janeiro, São Paulo).

Remarks. HINCKS (1956) recorded this species from Peru (Tinjo [= Tingo] Maria, v.1938, F. Tippmann) but noted that the data may be erroneous. Based on the available material, $C$. sexpustulata is primarily distributed in eastern Brazil and thus very unlikely in Peru. The specimen must have been mislabelled, and $C$. sexpustulata is removed from the fauna of Peru.

\section{*Cyrtonota thalassina (Boheman, 1850)}

Published records. Barueri; Cantareira (Borowiec 1996); São RoqueSitio Itatuba (Borowiec 2002); 'A Arusa [=Arujá]'; São Paulo (BorowIEC \& TAKIZAWA 2011).

Additional records. BRAZIL: São Paulo: São Paulo Capital, 17.ii.1912, 1 spec., 25.ii.1912, 1 spec., G. E. Bryant leg. (BMNH), 23.i.1968, 4 spec., J. Bick leg. (KVSC); Serra de Bocaina, 1500 m, xi.1965, 1 spec. (NHMB); Vale do Rio Prado, xii.1898, 1 spec., E. Gounelle leg. (BMNH).
Distribution. Argentina, Brazil (Distrito Federal, Espírito Santo, Minas Gerais, Pará, Paraná, Rio de Janeiro, Rio Grande do Sul, Santa Catarina, São Paulo), and Paraguay.

\section{*Cyrtonota vulnerata (Boheman, 1850)}

New record. BRAZIL: São Paulo: without further data, $1 \stackrel{\jmath}{\partial}$ J. Mráz leg. (NMPC).

Distribution. Brazil (Minas Gerais, Rio de Janeiro). New to Minas Gerais and São Paulo.

Remarks. SIMÕES \& MonNÉ (2011) published new faunistic records of Cyrtonota similata (Boheman, 1850) from Manhumirim (Minas Gerais), Itatiaia, and Resende-Serrinha do Alambari (both in Rio de Janeiro). All four specimens were deposited in the National Museum in Rio and thus destroyed by the fire in 2018. Nevertheless, the authors provided a colour photograph of one representative, which quite clearly shows that the species was misidentified as $C$. vulnerata. The latter is characterized by semiopaque and velvet-like dorsum due to finer punctation and by high postscutellar tubercle, while $C$. similata has very low postscutellar tubercle and shiny and coarsely punctate elytra. It is also considerably smaller than $C$. vulnerata but unfortunately, no scale was provided. Consequently, all three records must be transferred to $C$. vulnerata and removed from $C$. similata, which is thus known only from the type locality - the state of Bahia (BOHEMAN 1850) as no other records have been published for it. Cyrtonota vulnerata was so far reported only from Rio de Janeiro (FLINTE et al. 2009).

\section{Cyrtonota zikani (Spaeth, 1932)}

Published records. Campos do Jordão (SpaEth 1932a, Borowiec 1996).

Distribution. Brazil (Rio de Janeiro, Rio Grande do Sul, São Paulo).

\section{Hilarocassis albida (Germar, 1823)}

Published record. São Paulo (SIMÕES \& MONNÉ 2008).

Distribution. Brazil (Minas Gerais, Rio de Janeiro, São Paulo) and Paraguay.

\section{*Hilarocassis evanida (Boheman, 1850)}

New record. BRAZIL: São PAulo: Campinas, 1 spec. (BMNH).

Distribution. Argentina, Bolivia, Brazil (Paraná), Colombia, ? Mexico, Paraguay, and Peru. New to São Paulo.

\section{Mesomphalia gibbosa (Fabricius, 1781)}

New record. BRAZIL: SÃo PAULo: without further data, 1 spec. (BMNH).

Distribution. Brazil (Espírito Santo, Minas Gerais, Rio de Janeiro). New to São Paulo.

\section{Mesomphalia nudoplagiata Spaeth, 1901}

Published record. Itanhaém (SIMõES \& MONNÉ 2014).

Distribution. Brazil (Espírito Santo, Minas Gerais, São Paulo). 
*Mesomphalia sublaevis Boheman, 1850

Published records. Campos do Jordão; Cantareira (SIMõEs \& MonnÉ 2014).

Distribution. Brazil (Minas Gerais, Rio Grande do Sul, Rio de Janeiro, Santa Catarina, São Paulo).

\section{*Mesomphalia turrita (Illiger, 1801)}

Published records. Santos; São Paulo (BorowiEC 1996, as M. sexmaculata Boheman, 1850); Cubatão (Borowiec 2009); São Paulo (BorowiEC \& TAKIZAWA 2011, as M. sexmaculata); Campos do Jordão; Cubatão; Joinville; Mogi da Cruzes; São Paulo; Serra da Cantareira; Serra da Bocaina (SIMÕES \& MONNÉ 2014).

Additional records. BRAZIL: São Paulo: Alto da Serra, 5.iii.1912, 2 spec., 9.iii.1912, 3 spec., G. E. Bryant leg. (BMNH); Santos, 22.xi.1911, 1 spec., 18.iii.1912, 1 spec., 20.iii.1912, 1 spec., 16.iv.1912, 3 spec., 20.iv.1912, 1 spec., 25.iv.1912, 2 spec., G. E. Bryant leg. (BMNH)

Distribution. Argentina and Brazil (Espírito Santo, Goiás, Paraná, Rio de Janeiro, Santa Catarina, São Paulo).

\section{*Nebraspis corticina (Boheman, 1850)}

New records. São Paulo: without further data, $1 \lesssim 1$ ๆ J. Mráz leg. (NMPC), 1 spec. (BMNH); Butantan, iii.1934, 1 spec. (NHMB).

Distribution. Argentina, Brazil (Goiás), and Paraguay. New to São Paulo.

\section{Omaspides (s. str.) iheringi (Spaeth, 1909)}

Published record. Franca (SPAETH 1909).

Distribution. Brazil (Rio de Janeiro, São Paulo) and Peru. Remarks. This species was recorded from Madre de Dios Department in Peru by BorowIEC (2009). I did not have the opportunity to study the respective material; however, the distribution is suspicious as no other species of Omaspides Chevrolat, 1836 has such a vicariant distribution in eastern Brazil and the interior Amazon. In this case, the locality data are almost certainly correct as the material was provided directly by the collector, thus it would be desirable to compare the material from Peru to the holotype of $O$. iheringi to confirm its identity.

\section{Omaspides (s. str.) pallidipennis (Boheman, 1854)}

Published records. Indiana; São Sebastião (Buzzi 1988); Serra do Mar-Est. Biol. Boracéia, 50 km SE of Mogi das Cruzes (Borowiec 2009); 'Arusa [= Arujá]' (BorowIEC \& TAKIZAWA 2011).

Additional record. BRAZIL: SÃo Paulo: Alto da Serra, 28.ii.1912,

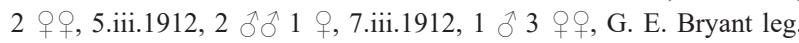
$(\mathrm{BMNH})$.

Distribution. Brazil (Espírito Santo, Goiás, Rio Grande do Sul, Minas Gerais, Paraná, Rio de Janeiro, Santa Catarina, São Paulo).

\section{*Omaspides (s. str.) tricolorata (Boheman, 1854)}

Published records. Cantareira; São Paulo (BorowIEC 1996); 'Arusa [= Arujá]' (Borowiec \& TAKIZAWA 2011).

Distribution. Brazil (Minas Gerais, São Paulo).
*Omaspides (Paromaspides) brunneosignata (Boheman, 1854)

Published record. Engenheiro Coelho (SIMõES \& Monné 2008).

Distribution. Brazil (Minas Gerais, São Paulo).

*Omaspides (Paromaspides) squalida

(Boheman, 1854)

Published record. Bocaina (BorowIEC 1996).

Distribution. Brazil (Rio de Janeiro, São Paulo).

Paraselenis (Spaethiechoma) aulica (Boheman, 1854)

Published record. Bocaina (BorowIEC 1996).

Distribution. Brazil (Rio de Janeiro, São Paulo).

Paraselenis (Spaethiechoma) collata (Boheman, 1854)

Published record. 'Mendes [= collector; thus, São Paulo only]' (BoROWIEC 1996).

Distribution. Brazil (Minas Gerais, São Paulo).

Remarks. Borowiec (1996) published material labelled 'Sao Paulo Mendes' and interpreted Mendes as the name of locality. However, it is most likely a name of collector rather than locality (L. Borowiec, pers. comm. 2020) and thus, the record is here interpreted as São Paulo only.

\section{Paraselenis (Spaethiechoma) decipiens}

(Boheman, 1854)

Published record. Peruíbe (Buzzi 1988).

Distribution. Brazil (Minas Gerais, Rio de Janeiro, São Paulo).

*Paraselenis (Spaethiechoma) dichroa (Germar, 1823)

Published records. Piracicaba (Toledo Piza 1968); Barueri; Campinas; Cantareira; São Paulo (BorowIEC 1996); São Roque-Sitio Itatuba (Borowiec 2002).

Additional record. BRAZIL: São Paulo: Campinas, $1 q(\mathrm{BMNH})$, v.1883, 2 ㅇ, A. Fry leg. (BMNH).

Distribution. Argentina, Brazil (Minas Gerais, Paraná, São Paulo), and Paraguay.

Paraselenis (Spaethiechoma) flava (Linnaeus, 1758)

Published record. Saltinho (LoPes et al. 2016).

Distribution. Argentina, Bolivia, Brazil (Paraíba, São Paulo), Guyana, and Paraguay.

\section{Paraselenis (Spaethiechoma) transversalis (Boheman, 1854)}

Published record. Campinas (BorowIEC 1996).

Distribution. Brazil (São Paulo).

*Stolas acuta (Boheman, 1850)

New records. BRAZIL: São PAulo: without further data, 1 \ $9 \circ$, J. Mráz leg. (NMPC), 1 spec. (BMNH). 
Distribution. Brazil (Rio de Janeiro). New to São Paulo.

Stolas aenea (Olivier, 1790)

New record. BRAZIL: São PAULO: without further data, 2 spec, Weir leg. (BMNH).

Distribution. Brazil (Minas Gerais, Rio de Janeiro, Santa Catarina), and Paraguay. New to São Paulo.

Remarks. BoRowIEC (1996) recorded several species, which are common in southern part of South America, as new to French Guiana based on old material labelled as from Bas Maroni. Borowiec \& Moragues (2005) critically evaluated all records published from French Guiana and removed four of these species published from Bas Maroni as their occurrence in French Guiana is very improbable; however, $S$. aenea remained in the review as an accepted record. However, occurrence of $S$. aenea in French Guiana is also dubious as the species has not been recorded in NE Brazil, and it is removed from the species distribution.

\section{*Stolas antiqua (Sahlberg, 1823)}

Published record. São Paulo (BorowIEC 1996).

Distribution. Argentina, Brazil (Mato Grosso, Paraná, Rio de Janeiro, Rio Grande do Sul, Santa Catarina, São Paulo), and Paraguay.

\section{*Stolas areolata (Germar, 1823)}

Published records. Barueri; Bocaina; Butantan; Cantareira; São Paulo (BorowIEC 1996); Jundiaí; São Roque-Sitio Itatuba (BorowIEC 2002); Serra do Japi (NogueIra-DE-SÁ \& Vasconcellos-Neto 2003a,b); Itu (Borowiec 2009).

Additional records. BRAZIL: São Paulo: São Paulo Capital, v.1883, 3 spec., A. Fry leg. (BMNH), 17.ii.1912, 6 spec., 20.ii.1912, 12 spec., 24.ii.1912, 1 spec., 26.ii.1912, 9 spec., G. E. Bryant leg. (BMNH).

Distribution. Argentina and Brazil (Minas Gerais, Paraná, Rio de Janeiro, São Paulo).

*Stolas brevicuspis (Spaeth, 1923)

Published record. São Paulo (SPAETh 1923).

Distribution. Brazil (Rio de Janeiro, São Paulo).

\section{Stolas brunni (Spaeth, 1905)}

Published record. São Paulo (SPAETH 1905).

Distribution. Brazil (São Paulo).

\section{*Stolas chalybaea (Germar, 1823)}

Published records. Piracicaba (Toledo Piza 1968); Bocaina (HAITlinGER 1991); Cantareira; São Paulo (BorowIEC 1996); Parque Estadual Intervales (NogueIRA-DE-SÁ \& Trigo 2002); Serra do Japi (NogueIRA -DE-SÁ \& VASCONCELlos-Neto 2003a,b).

Additional records. BRAZIL: São PAULo: Sao Paulo Capital, v.1883, 3 spec., A. Fry leg. (BMNH), 21.ii.1912, 1 spec., G. E. Bryant leg. (BMNH), 30.iv.1949, 1 spec. (NHMB), 5.ii.1967, 2 spec., J. Bick leg. (KVSC).

Distribution. Argentina, Brazil (Pará, Paraná, Rio Grande do Sul, Santa Catarina, São Paulo), and Paraguay.

\section{*Stolas conspersa (Germar, 1823)}

Published record. Campos do Jordão (BorowiEC 1996). Additional record. BRAZIL: São Paulo: Campinas-Fazenda Souzas, 30.x.1994, 1 spec., W. Wittmer leg. (NHMB).

Distribution. Brazil (Amazonas, Ceará, Distrito Federal, Espírito Santo, Mato Grosso, Minas Gerais, Pará, Rio de Janeiro, São Paulo) and Paraguay.

\section{Stolas deleta (Boheman, 1850)}

Published record. São Paulo (BorowIEC 1996).

Additional record. BRAZIL: São Paulo: Santos, 22.xi.1911, $1 \mathrm{spec}$., 3.iii.1912, 1 spec., 29.iii.1912, 1 spec., G. E. Bryant leg. (BMNH).

Distribution. Brazil (Minas Gerais, Rio Grande do Sul, São Paulo).

\section{*Stolas iheringi (Spaeth, 1913)}

Published records. Alto da Serra (SpAEth 1913); Mato do Governo (BOROWIEC 1996).

Distribution. Brazil (São Paulo).

\section{Stolas imperialis (Spaeth, 1898)}

Published record. São Paulo (Borowiec 1996).

Distribution. Brazil (Minas Gerais, Santa Catarina, São Paulo).

\section{Stolas implexa (Boheman, 1850)}

Published record. Cantareira (BorowiEc 1996).

Distribution. Argentina, Brazil (Minas Gerais, Paraná, Rio de Janeiro, Rio Grande do Sul, Santa Catarina, São Paulo), and Uruguay.

\section{*Stolas impluviata (Boheman, 1850)}

Published record. São Paulo (BorowIEC \& PoMORSKa 2009). Additional record. BRAZIL: São Paulo: Cantareira, $1 \mathrm{spec}$., Monte leg. (BMNH).

Distribution. Brazil (Rio de Janeiro, Santa Catarina, São Paulo).

\section{*Stolas lacordairei (Boheman, 1850)}

Published records. São Paulo (Borowiec 1996, BuZzi \& MiYaZaKI 1999).

Distribution. Argentina, Brazil (Paraná, Rio Grande do Sul, Santa Catarina, São Paulo), Chile, Paraguay, and Uruguay.

Remarks. All specimens collected by J. Mráz belong to a uniformly metallic blue-green form while the typical form has red elytra with numerous metallic spots.

\section{Stolas lenis (Boheman, 1850)}

Published records. Monte Azul (Borowiec 1996); São Paulo (BoROWIEC \& POMORSKA 2009).

Distribution. Brazil (São Paulo). 
*Stolas lineaticollis (Boheman, 1850)

$$
\text { (Figs 13-17) }
$$

Mesomphalia lineaticollis Boheman, 1850: 333. Type locality: 'Brasilia'. Mesomphalia silaceipennis Boheman, 1862: 169. Type locality: 'Brasilia'; syn. nov.

Type material examined. Mesomphalia lineaticollis: LECTOTYPE (designated by Borowiec (1999)): $\hat{\partial}$, pinned, 'Brasil [w, p, s] \| M. Wien [w, p, s] \| Type. [w, p, s] || LECTOTYPE | des. L. Borowiec [r, p, cb] || NHRS-JLKB | 000022713 [w, p, cb]' (NHRS). Paralectotypes: 1 q, pinned, 'Brasil [w, p, s] || Schh [w, p, s] || Type. [w, p, s] || PARALECTOTYPE | des. L. Borowiec [r, p, cb] || NHRS-JLKB | 000022714 [w, p, cb]' (NHRS); 1 ;, pinned: 'Brasil [w, p, s] || Bhn. [w, p, s] || PARALECTOTYPE | des. L. Borowiec [r, p, cb] || NHRS-JLKB | 000022715 [w, p, cb]' (NHRS); 1 \%, pinned: 'Brasil [w, p, s] || M. Wien [w, p, s] || PARALECTOTYPE | des. L. Borowiec [r, p, cb] || NHRS-JLKB | 000022716 [w, p, cb]' (NHRS).

Mesomphalia silaceipennis: SYNTYPE: d, 'pinned, Brasil [w, p, s] || Dohrn [w, hw, s] || Type. [w, p, s] || NHRS-JLKB | 000022641 [w, p, cb]' (NHRS).

Additional type material examined for comparative purpose. Mesomphalia augur. HolotyPe: + , pinned: 'E. Coll | Chev'. [w, p, cb] || 78 [g, p, s] || Type [w, p, s, circle label with red frame] || N. | Picoffer [w, hw, s] || Mesomp | augur | Bhn | Bras [w, hw, s, Chevrolat's hw] || 67.56 [w, p, s]' (BMNH).

Additional material examined. São PaULo: without further data, $9 \curvearrowright 20$ †, J. Mráz leg. (NMPC); Campinas, v.1883, 2 spec., A. Fry leg. (BMNH). Published records. Indiana; São Paulo (BuzzI 1988); Cantareira (BoROWIEC 1996).

Distribution. Argentina, Bolivia, Brazil (Espírito Santo, Minas Gerais, Rio de Janeiro, Santa Catarina, São Paulo), and Peru.

Remarks. BorowIEC (1999) synonymized Stolas silaceipennis with $S$. augur without further details, but presumably based on the note in Spaeth's unpublished manuscript Cassidinae der Welt stating that 'M[esomphalia]. silaceipennis is presumably hardly different'. BOHEMAN (1862) stated that Mesomphalia silaceipennis had the same size and shape as M. lineaticollis and, comparing the primary descriptions of the two species, that the main differences were in body colouration, i.e., yellow pronotum with a medial black stripe continuing along the suture to the apex of elytra in $M$. lineaticollis, and red-brown pronotum without a medial dark stripe but elytra with a brown stripe along the anterior third of the suture in $M$. silaceipennis. Mesomphalia augur was described from the collection of Chevrolat presumably based on a single specimen (BOHEMAN 1857) which was never studied by subsequent workers on Cassidinae; as a result, $M$. augur was only cited in catalogues (see BorowIEC 1999). I have studied types of all three taxa involved, and in my opinion S. silaceipennis differs from $S$. lineaticollis only in colour, otherwise the shape of elytra and pronotum, convexity of elytra, and their punctation are identical. Even the syntype of S. silaceipennis has a slightly indicated medial stripe in the anterior third of pronotum, and it is well known that small species of Stolas Billberg, 1820, associated with Convolvulaceae, are very variable in colouration. This is nicely demonstrated by the series of specimens collected by Mráz in São Paulo which contain examples nearly identical to the types of both, S. lineaticollis (Fig. 13) and S. silaceipennis (Fig. 17), with numerous intermediate forms (e.g. Figs 14-16) having a broad black medial stripe on brown pronotum or yellow pronotum nearly without a medial stripe; some of the yellow specimens have suture in the postscutellar third more or less brownish. Finally, S. lineaticollis was reported as feeding on Convolvulaceae by BuzzI (1994). Therefore, I hereby synonymize $S$. silaceipennis with $S$. lineaticollis.

Stolas augur also belongs to this group of small species presumably feeding on Convolvulaceae but it has narrower body and smaller but denser punctation of elytra in comparison to $S$. lineaticollis; thus, I consider it a valid species. So far, I have not seen any other specimen matching it and thus it is known only from the holotype specimen.

The material collected by Mráz was identified by Spaeth as S. lineaticollis and S. silaceipennis, and he considered all specimens with brown pronotum as belonging to $S$. silaceipennis, not distinguishing whether they had a medial black stripe on pronotum or not. On the other hand, this character was used as critical to separate $S$. lineaticollis and $S$. augur (incl. S. silaceipennis) in his manuscript of Cassidinae der Welt. Probably most, if not all specimens identified by Spaeth as $S$. silaceipennis are in accordance with its type but do not belong to $S$. augur.

\section{Stolas modica (Boheman, 1850)}

Published record. Engenheiro Coelho (SIMõES \& MonNÉ 2008).

Distribution. Brazil (Bahia, Espírito Santo, Rio de Janeiro, Santa Catarina, São Paulo).

\section{*Stolas oblita (Boheman, 1850)}

Published record. São Paulo Capital (BorowiEc 2009).

Distribution. Brazil (Pará, Santa Catarina, São Paulo).

\section{Stolas paulista (Spaeth, 1935)}

Published records. Santos; São Paulo (SPAETH 1935).

Distribution. Brazil (Paraná, São Paulo).

\section{*Stolas perfuga (Spaeth, 1926)}

$$
\text { (Fig 18) }
$$

Published record. São Paulo (SPAETH 1926c).

Type material examined. SyNTYPE (Fig. 18): 1 , pinned, 'SAÕ PAULO | BRAS. MRÁZ LGT. | MUS.PRAGENSE [w, p, cb, bf] || Pseudomesomphal. | perfuga cotyp. [hw by Spaeth] | Spaeth determ. [w, p, cb] || SYNTYPUS | Pseudomesomphalia | perfuga | Spaeth, 1926 | L. Sekerka des. 2020 [r, p, cb, bf]' (NMPC).

Distribution. Brazil (São Paulo).

Remarks. SPAETH (1926c) described this species based on three specimens collected by Jaro Mráz in São Paulo. He did not fix the type (in the meaning of holotype) in the original publication, hence all three are syntypes. The two remaining specimens were retained by F. Spaeth for his collection.

\section{*Stolas plagicollis (Boheman, 1850)}

Published records. Guaruja near Santos (BorowIEC 1996); São Paulo (Borowiec \& PoMorsKa 2009).

Additional record. BRAZIL: São PAulo: Cerqueira César, i.1889, 1 1 †, E. Gounelle leg. (BMNH).

Distribution. Argentina, Brazil (Bahia, Minas Gerais, Paraná, Rio Grande do Sul, São Paulo), and Paraguay. 


\section{*Stolas redtenbacheri (Boheman, 1850)}

Published records. São Paulo (Haitlinger 1991); Campinas (BoROWIEC 1996); São Roque-Sitio Itatuba (BorowIEC 2002); Rio Claro (Lopes et al. 2016).

Additional record. BRAZIL: São PAulo: Ilha de Santo Amaro-near
Santos, 14.iv.1912, 1 spec., G. E. Bryant leg. (BMNH).

Distribution. Argentina, Brazil (Mato Grosso, Minas Gerais, Paraná, Rio de Janeiro, Rio Grande do Sul, São Paulo), and Paraguay.
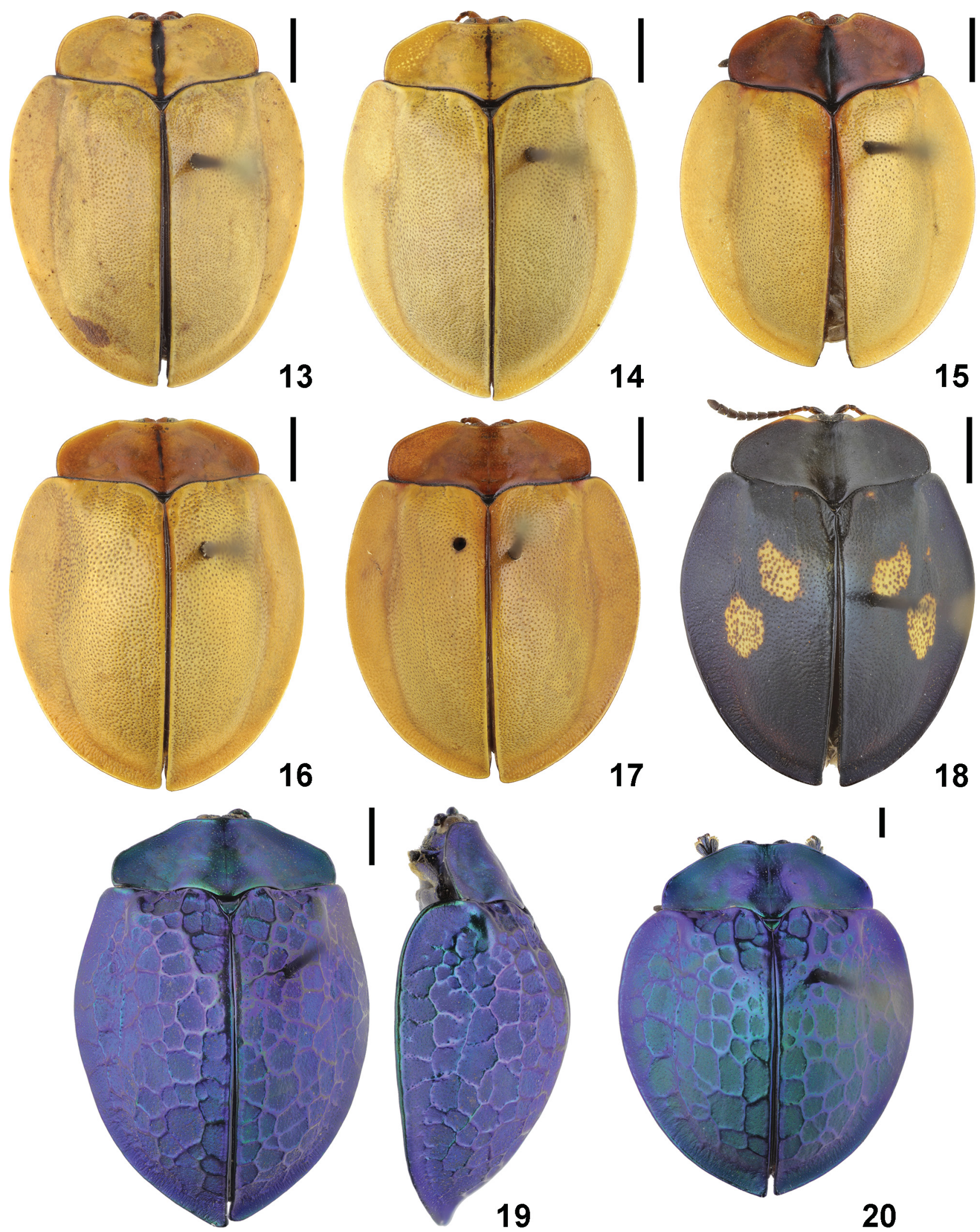

Figs 13-20. Dorsal aspects. 13-17 - Stolas lineaticollis (Boheman, 1850), variability, all collected by J. Mráz; 18 - Stolas perfuga (Spaeth, 1926), syntype, $+; 19-20-$ Stolas selecta (Spaeth, 1928), syntypes: $19-+; 20-$. Scale bars $=2 \mathrm{~mm}$. 
*Stolas selecta (Spaeth, 1928)

(Figs 19-20)

Published records. Amparo; São Paulo (SPAETH 1928).

Type material examined. SYNTYPES: 1 (Fig. 19), pinned, 'SAÕ PAULO | BRAS. MRÁZ LGT. | MUS.PRAGENSE [w, p, cb, bf] || Pseudomesomphal. | selecta $\mathrm{m}$. + [hw by F. Spaeth] | Spaeth determ. [w, p, cb] || COTYPUS [pink, p, cb]' (NMPC); 1 (Fig. 20), pinned, 'Sao PAULO; Mráz | legit. Brasilia | mus. R. BOH, [w, p, cb] || Pseudomesomphal. | selecta m. cot. [hw by F. Spaeth] | Spaeth determ. [w, p, cb]' (NMPC); 2 ôे 3 우, pinned, 'SAÕ PAULO | BRAS. MRÁZ LGT. | MUS.PRAGENSE [w, p, cb, bf]' (NMPC); 2 + $ᄋ$, pinned, 'SAO PAULO | Br. MRÁZ [w, p, cb]' (NMPC); 3 đô, pinned, 'Sao PAULO; Mráz| legit. Brasilia| mus. R. BOH, [w, p, cb]' (NMPC); 1 ' pinned, 'Sao PAULO; Mráz| legit. Brasilia | mus. R. BOH, [w, p, cb] || Pseudomesomphal. | retis Wag. [hw by F. Spaeth] | Spaeth determ. [w, p, cb]' (NMPC). Each specimen was provided with an additional label: 'SYNTYPUS | Pseudomesomphalia | selecta | Spaeth, 1928 | L. Sekerka des. 2020 [r, p, cb, bf]'.

Additional record. BRAZIL: São Paulo: São Paulo Capital, v.1883, 6 spec., A. Fry leg. (BMNH).

Distribution. Brazil (Mato Grosso, Rio de Janeiro, Rio Grande do Sul, Santa Catarina, São Paulo).

Remarks. SPAETH (1928) described the species based on numerous specimens, mainly collected by J. Mráz, but did not mention the precise number of specimens nor he did mention one particular specimen being the type. In NMPC there were two series, one identified as $S$. selecta while only two specimens were labelled with Spaeth's original label as cotypes, and another identified as $S$. retis (Wagener, 1881). All specimens have the same morphology and differ only in colour. I assume that Spaeth identified the entire series as $S$. retis at first and later on, when he decided that it was actually a new species, he considered the entire series in NMPC to be part of the type series. Therefore, I consider all specimens part of the type series and they all are syntypes.

The series display variability in colouration and size of reticulation of elytra. Six specimens are green-blue (one of them being the labelled male syntype, Fig. 20), one specimen is blue-green (the labelled female syntype, Fig. 19), four are dark blue (three of them were identified as $S$. retis), and finally two specimens are nearly black with obscure blue tint.

\section{*Stolas sexplagiata (Boheman, 1850)}

Published records. Bosque da Saúde; Cantareira (BorowIEc 1996).

Distribution. Brazil (Rio de Janeiro, São Paulo).

\section{Stolas sexsignata (Boheman, 1850)}

New record. BRAZIL: São PAuLo: without further data, 1 spec. (ZSMC).

Distribution. Brazil (Paraná). New to São Paulo.

\section{Stolas sommeri (Boheman, 1850)}

New record. BRAZIL: São PAulo: Alto da Serra, 7.iii.1912, 1 spec., G. E. Bryant leg. (BMNH).

Distribution. Brazil (Paraná) and Paraguay. New to São Paulo.

\section{*Stolas subreticulata (Boheman, 1850)}

New record. BRAZIL: São PAulo: without further data, 1 , J. Mráz leg. (NMPC).
Distribution. Argentina, Brazil (Rio de Janeiro). New to São Paulo.

\section{Stolas tumulus (Boheman, 1850)}

Published record. Peruíbe (BorowIEC 1996).

Distribution. Brazil (Santa Catarina, São Paulo).

\section{Zatrephina lineata (Fabricius, 1787)}

Published record. Bocaina (BorowIEC 1996).

Distribution. Brazil (Amapá, Pará, Paraíba, Pernambuco, Rio de Janeiro, São Paulo), Colombia, French Guiana, and Paraguay.

\section{Tribe Omocerini}

\section{Canistra (s. str.) irrorata (Guérin-Méneville, 1844)}

Published records. Santo Amaro (VIANA 1964a); Monte Azul (BoROWIEC 1996).

Distribution. Argentina, Bolivia, Brazil (Amazonas, Goiás, Mato Grosso, Mato Grosso do Sul, Pará, São Paulo), and Paraguay.

\section{* Canistra (Canistrella) rubiginosa (Guérin-Méneville, 1844)}

Published records. Alto da Serra; Barueri; Campos do Jordão; Congonhas; Jundiaí; Mogi das Cruzes; Osaco; São Paulo; São Paulo-Ipiranga; Tres Pedras (Viana 1964a); Piracicaba (Toledo Piza 1968); Barueri; Butantan; Cantareira; Teodoro Sampaio (Borowiec 1996); São RoqueSitio Itatuba (BorowIEC 2002).

Additional records. BRAZIL: São PAulo: São Paulo Capital, 16.ii.1912, 1 spec., G. E. Bryant leg. (BMNH).

Distribution. Argentina, Brazil (Bahia, Espírito Santo, Goiás, Minas Gerais, Paraná, Rio de Janeiro, Rio Grande do Sul, Santa Catarina, São Paulo), Paraguay, and Uruguay.

Carlobruchia (Smodingonota) carbonaria (Klug, 1829)

Published record. Agudos (Viana 1964a).

Distribution. Argentina, Bolivia, Brazil (Goiás, 'Mato Grosso', Minas Gerais, Rio Grande do Sul, São Paulo), Paraguay, and Uruguay.

\section{Cassidinoma denticulata (Boheman, 1850)}

Published record. Iraguare (Fernandes \& Borowiec 2013).

Distribution. Argentina, Bolivia, Brazil (Bahia, Goiás, Mato Grosso, Mato Grosso do Sul, Minas Gerais, São Paulo), and Paraguay.

\section{*Cyclosoma (Dolichotoma) fuscopunctatum (Spaeth, 1919)}

Published record. São Paulo (BorowiEc 1996). Additional records. BRAZIL: SÃo PAULo: without further data, 16 spec., J. Mráz leg. (NMPC, 3 LSPC); Alto da Serra, ii.1928, 1 spec. (BMNH).

Distribution. Brazil (Santa Catarina, São Paulo). Remarks. Boheman (1850) described Dolichotoma clypeata presumably based on a single specimen originally 
from the collection of Dejean deposited in the collection of Mannerheim. The species was listed only in catalogues since its description. SpaEth (1919) described $D$. fuscopunctata and stated that it was of the same size and shape as D. clypeata and differing in colouration of elytra, denser and coarser punctation of the elytra, shallower emargination of pronotum, black colouration of ventral side including larger parts of the legs. These characters, except for the punctation of elytra, are very variable in the species of Dolichotoma Hope, 1839 (nowadays a subgenus of Cyclosoma Guérin, 1835). It is nicely demonstrated by the series collected by J. Mráz as almost each of the twelve specimens has a slightly different colour. Most of them are similar to the colouration of $C$. fuscopunctatum with more or less distinct dark patches on the explanate margin of elytra, but three specimens have colouration of $C$. clypeatum which should be red with black spots only on the disc of elytra with uniformly red explanate margin. In BMNH I found two specimens (ex coll. J. Baly) identified by Boheman as C. clypeatum, and these perfectly match the specimens collected by $\mathrm{J}$. Mráz. On the other hand, Boheman certainly identified these specimens after the description of C. clypeatum (probably during 1855-1861) and because the type is not in his collection, he most likely did not compare the specimens, so there is a chance he misidentified the two specimens from Baly, and they belong to C. fuscopunctatum. If they are truly conspecific with $C$. clypeatum, then C. fuscopunctatum is a synonym of it. Because I was not able to locate and thus study the type of C. clypeatum, I do not propose the synonymy yet.

BOROWIEC \& TAKIZAWA (2011) recorded this species from the Peruvian Amazon (Nautá near Iquitos), and I find this record doubtful. All species of the subgenus Dolichotoma Hope, 1839 are distributed with certainty in eastern Brazil; other records must be taken as dubious unless confirmed by new and reliable material. Obviously, part of the material published by BOROWIEC \& TAKIZAWA (2011) must have been mislabelled because the distribution would be very unlikely as a species presumably restricted to the Atlantic forest was recorded also from the Peruvian Amazon. There are such species of Cassidinae but they usually have a much wider distribution through most of South America. Therefore, C. fuscopunctatum is removed from the fauna of Peru.

\section{Cyclosoma (Dolichotoma) germari (Spaeth, 1913)}

Published records. Alto da Serra; Campos da Serra-Rio M'boy Guassú; Interlagos; Jundiaí; Paranapiacaba; Rio da Serra; São Paulo-Ipiranga (VIANA 1964a); Cantrareira (BOROWIEC 1996).

Additional records. BRAZIL: São Paulo: Vale do Rio Prado, xii.1898, 2 spec., E. Gounelle leg. (BMNH).

Distribution. Brazil (Espírito Santo, Minas Gerais, Rio de Janeiro, Santa Catarina, São Paulo) and Uruguay.

Remarks. Viana (1964a) recorded three specimens from Rio Juruá (which springs from the Ucayalí province in Peru and flows through the states of Acré and Amazonas, where it flows into the Amazon River). BorowIEC (1996) also published one specimen from the Kourou River in French
Guiana. I consider these records doubtful, being off the distributional limits of $C$. germari, and the specimens were probably mislabelled. Accordingly, the species is removed from the fauna of French Guiana and the state of Amazonas.

\section{Cyclosoma (Dolichotoma) mitior (Boheman, 1850)}

Published records. Alto da Serra; Itarare; Ribeirao Pires (VIAna 1964a).

Distribution. Brazil (Minas Gerais, São Paulo, ?Pará).

Remarks. VianA (1964a) recorded one specimen from 'Amazonas, Itautuba'. It is most likely Itaituba on the Amazon River in the state of Pará. No other specimen was recorded from the Amazon. As stated under C. germari I find the occurrence of species of Dolichotoma in other areas except for eastern Brazil dubious and caused either by mislabelling of specimens or misidentifications. Therefore, C. mitis is removed from the fauna of the state Amazonas; moreover, the record, if accepted, should be scored to the state of Pará.

\section{Omocerus (Nebroma) gibberus (Boheman, 1850)}

Published records. Osasco; São Paulo-Ipiranga (VIAna 1964a).

Distribution. Brazil (Minas Gerais, Paraná, São Paulo).

Omocerus (Nebroma) humerosus (Spaeth, 1913)

Published records. São Paulo (SPAETH 1913); Franca (VIANA 1964a); Rio Claro (BorowIEC \& TAKIZAWA 2011).

Distribution. Brazil (Goiás, Mato Grosso do Sul, Minas Gerais, Paraná, São Paulo).

Omocerus (Nebroma) klugi (Spaeth, 1913)

Published record. São Paulo (BoHEMAn 1857, as O. antiquus (Klug, 1829)).

Distribution. Argentina, Bolivia, Brazil (Mato Grosso, Minas Gerais, Paraná, Rio Grande do Sul, Santa Catarina, São Paulo), and Paraguay.

\section{Omocerus (Platytauroma) cornutus (Boheman, 1850)}

New record. BRAZIL: São Paulo: without further data, 1 spec., Weir leg. (BMNH).

Distribution. Brazil (Bahia, Rio de Janeiro). New to São Paulo.

\section{*Omocerus (Platytauroma) truncatus (Boheman, 1850)}

Published records. São Paulo (SoAres 1962); Campo Grande; Cantareira; Jabaquara; Jaguaratinga; Jupuvura; Juquiá; Juquiá-Fazenda Poço Grande; Santo Amaro; São Paulo; São Paulo-Ipiranga (VIANA 1964a); Cantareira; São Paulo (BorowIEc 1996).

Distribution. Argentina, Brazil (Espírito Santo, Minas Gerais, Paraná, Rio de Janeiro, Rio Grande do Sul, Santa Catarina, São Paulo), and Paraguay.

Remarks. Records from Brazil: Amazonas: Manaus (Couto SoAres 1962), Bolivia: Chiquitos (VIANA 1964a), Colombia: Ocaña (HINCKs 1956), and French Guiana: Cayenne (CHABOо 2002) are dubious and occurrence in 
these countries or states, respectively, was based on misidentifications or erroneously labelled specimens. Species of the subgenus Platytauroma Spaeth, 1913 are distributed in south-eastern part of South America from the state of Bahia (Brazil) to northern Argentina (Misiones).

*Polychalca (s. str.) punctatissima (Wolf, 1818)

Published record. São Paulo (BorowIEC 1996).

Distribution. Argentina and Brazil (Bahia, Ceará, Goiás, Minas Gerais, Pará, Paraíba, Pernambuco, Rio de Janeiro, Rio Grande do Norte, Rio Grande do Sul, São Paulo).

\section{*Polychalca (Desmonota) platynota (Germar, 1823)}

Published records. Barueri; Juquiá-Fazenda Poço Grande; Mogi das Cruzes; Peruhybe; Registro (VIAna 1964a); Cantareira (BorowIEC 1996); Mogi das Cruzes-Fazenda Carmelita (BorowieC 2002).

Distribution. Argentina and Brazil (Distrito Federal, Minas Gerais, Rio de Janeiro, Santa Catarina, São Paulo).

Tribe Sceloenoplini

\section{Acentroptera basilica Thomson, 1856}

Published record. Campos do Jordão (Uhmann 1964).

Distribution. Argentina, Brazil (Bahia, Rio de Janeiro, Santa Catarina, São Paulo), French Guiana, and Paraguay.

\section{Acentroptera norrisii Guérin-Méneville, 1844}

Published record. São Paulo (STAINES 2014).

Distribution. Brazil (Paraná, Rio de Janeiro, Santa Catarina, São Paulo) and French Guiana.

\section{*Acentroptera pulchella}

(Guérin-Méneville, 1830)

Published record. Cachoeiro Paulista (Uhmann 1964).

Distribution. Brazil (Minas Gerais, Rio de Janeiro, Santa Catarina, São Paulo).

\section{Ocnosispa conicicollis (Baly, 1859)}

Published record. Município de Iporanga (Uhmann 1964).

Distribution. Brazil (Amazonas, Minas Gerais, Santa Catarina, São Paulo).

\section{Pseudispa brunni (Weise, 1910)}

Published record. Bocaina (Uhmann 1932).

Distribution. Brazil (Santa Catarina and São Paulo).

\section{*Pseudispa marginata (Guérin-Méneville, 1844)}

Published record. Barueri (Uhmann 1964).

Distribution. Brazil (Espírito Santo, Minas Gerais, Paraná, Rio Grande do Sul, São Paulo).
Sceloenopla bimaculaticollis (Pic, 1948)

Published record. São Paulo (Descarpentries \& ViLliers 1959).

Distribution. Brazil (São Paulo).

Remarks. In the original description, PIC (1948) mentioned 'Brésil' only; however, the type material also has São Paulo written on the labels, which was specified by DesCARPENTRIES \& VilLIERS (1959).

\section{Sceloenopla pretiosa (Baly, 1859)}

Published record. Santo Amaro; São Paulo-Ipiranga (Uhmann 1964); Salesópolis-Estação Biológica de Boracéia (CASARI \& QUEIROZ 2005).

Distribution. Argentina, Brazil (Bahia, Espírito Santo, Rio de Janeiro, Santa Catarina, São Paulo), and Paraguay.

\section{Sceloenopla rectelineta (Pic, 1929)}

Published records. Campinas (Costa et al. 1988, as $S$. af. bidens (Fabricius, 1792)); Campinas, Capivari, Jundiaí, Louveira, Paulínia, Piracicaba (LourEnÇÃo et al. 1991, as S. bidens); both misidentifications.

Distribution. Bolivia, Brazil (Ceará), and Peru. New to São Paulo.

Remarks. This species was previously reported under the name $S$. bidens; however, based on photograph published by LOURENÇÃo et al. (1991) it looks different and matches $S$. rectelineata; see also Remarks under $S$. bidens. It seems that $S$. rectelineta is distributed in seasonally dry habitats in the belt from Bolivia and southern Peru to São Paulo, although it has not been recorded from central Brazil formally, and probably extends along the Atlantic coast to Ceará (Serra de Baturité) assuming that Uhmann (1961) identified the respective material correctly.

Tribe Spilophorini

\section{Calyptocephala brevicornis Boheman, 1850}

Published record. São Paulo (BorowIEC 1996).

Distribution. Argentina, Brazil (Amazonas, Goiás, Mato Grosso, Minas Gerais, Paraná, Rio Grande do Sul, São Paulo), Colombia, Paraguay, and Venezuela.

\section{*Calyptocephala nigricornis (Germar, 1823)}

New record. BRAZIL: São Paulo: without further data, 1 spec., J. Mráz leg. (NMPC).

Distribution. Brazil (Paraná, Rio de Janeiro, Rio Grande do Sul, Santa Catarina). New to São Paulo.

\section{Oediopalpa basalis (Baly, 1859)}

Published record. Bocaina (Uhmann 1932).

Distribution. Brazil (Rio de Janeiro, Rio Grande do Sul, São Paulo).

\section{*Oediopalpa brunnea (Uhmann, 1943)}

New record. BRAZIL: São Paulo: without further data, 1 spec., J. Mráz leg. (NMPC).

Distribution. Brazil (Santa Catarina) and Paraguay. New to São Paulo. 
Remarks. This species is similar to O. nigripes Baly, 1859 which was also recorded from São Paulo but can be distinguished by stout body (vs. slender body in O. brunnea). Otherwise, the two taxa are very similar.

\section{*Oediopalpa caerulescens (Baly, 1875)}

New record. BRAZIL: São Paulo: without further data, 1 spec., J. Mráz leg. (NMPC).

Distribution. Brazil (Bahia, Minas Gerais) and Paraguay. New to São Paulo.

\section{Oediopalpa fulvipes Baly, 1859}

New record. BRAZIL: São PaULo: Amparo, 1 spec. (MNRJ; destroyed).

Distribution. Brazil. New to São Paulo.

Remarks. Unfortunately, the abovementioned specimen was destroyed during the fire in MNRJ. Nevertheless, I feel it is important to mention it as it is not only a new record for the state of São Paulo but also the first accurately labelled specimen of this species as $O$. fulvipes was described generally from Brazil. It represented a very characteristic species, and the specimen perfectly matched the holotype which I compared it to back in 2017.

\section{*Oediopalpa gibbula (Uhmann, 1948)}

Published record. Monte Alegre-Fazenda Santa Maria (Uhmann 1964).

Distribution. Brazil (Rio de Janeiro, Santa Catarina, São Paulo).

\section{Oediopalpa insecta (Uhmann, 1948)}

Published record. Salesópolis-Estação Biol. de Boraceia (Uhmann 1964).

Distribution. Brazil (Paraná, Rio Grande do Sul, Santa Catarina, São Paulo).

\section{Oediopalpa nigripes Baly, 1859}

Published records. São Paulo (WeISE 1905); Cantareria (Uhmann 1964).

Distribution. Brazil (Santa Catarina, São Paulo) and Paraguay.

\section{Oediopalpa pertyi (Guérin-Méneville, 1844)}

Published records. São Paulo (WeIse 1905); Indiana; Porto Cabral (UhMANn 1964).

Distribution. Argentina, Brazil (Ceará, Mato Grosso, Pará, Rio Grande do Sul, São Paulo), Colombia, Paraguay, and Venezuela.

\section{Oediopalpa sternalis (Weise, 1910)}

Published record. Juquiá (Uhmann 1964).

Distribution. Brazil (Espírito Santo, São Paulo).

Oediopalpa thoracica (Uhmann, 1930)

Published record. Morumbi (Uhmann 1964).

Distribution. Brazil (Santa Catarina, São Paulo) and French Guiana.

\section{Oediopalpa variolata Uhmann, 1960}

Published record. Alto da Serra (Uhmann 1960).

Distribution. Brazil (São Paulo).

Species excluded from the fauna of São Paulo (arranged in alphabetic order)

\section{Agroiconota subvittata (Boheman, 1855) (Cassidini)}

Distribution. Bolivia, Brazil (Goiás), and Peru.

Remarks. The occurrence in São Paulo was based on the type locality of $A$. urbanae which was erroneously synonymized with $A$. subvittata and recently revalidated (SEKERKA 2016); see also remarks under A. subvittata.

\section{Alurnus batesii Baly, 1864 (Alurnini)}

Published record. São Paulo (StaInes 2013).

Distribution. Brazil (Amazonas), Ecuador, and Peru. Remarks. Alurnus batesii is an Amazonian species distributed in the Upper Amazon Basin and is known to occur in Brazil, Ecuador and Peru (Uhmann 1957, Staines 2013). In Brazil, it has been reliably recorded only from the state of Amazonas and most specimens in collections are old and were collected either near Tefé (= Teffe or Ega in the past) or São Paulo de Olivença (Sekerka, unpubl. data). STAINES (2013) published a few specimens from the two previous localities and also two specimens with locality data only stating 'São Paulo'. I did not have the opportunity to study these specimens, but it is very unlikely that the species would occur in the state of São Paulo and I assume that the locality refers to São Paulo de Olivença. Therefore, $A$. batesii is removed from the fauna of São Paulo.

\section{Alurnus grossus Fabricius, 1775 (Alurnini)}

Published record. Campinas (Staines 2013).

Distribution. Brazil (Pará), Ecuador, French Guiana, Peru, and Suriname.

Remarks. Staines (2013) published one specimen from 'Campinas' in Brazil and the record was interpreted as being the well-known Brazilian municipality Campinas in the state of São Paulo (Sekerka 2020). However, $A$. grossus is distributed on the Guiana shield and within Brazil it has been reported only from the state of Pará (Fischer 1935, Staines 2013). Therefore, my previous interpretation is erroneous and Campinas (= plains or meadows) is a very common geographical name, used for hundreds of places in Brazil. The locality either refers to a different Campinas, whereabouts of which is unknown to me as no other data were provided, or it has been mislabelled. Consequently, A. grossus is removed from the fauna of São Paulo.

\section{Cephaloleia affinis Baly, 1859 (Imatidiini)}

Published record. São Paulo (STAINES \& GARCía-Robledo 2014).

Distribution. Bolivia, Brazil (Amazonas, Pará), Colombia, Ecuador, French Guiana, Guyana, Peru, Suriname, and Venezuela. 
Remarks. StAINES \& GARCÍA-RoBLEDO (2014) published an unknown number of specimens from São Paulo collected during August-September 1879 and deposited in USNM but they did not present any additional data. I assume that this specimen(s) was actually collected in São Paulo de Olivença in the state of Amazonas as I have seen numerous specimens with similar data in the collection of René Oberthür in MNHN. Also, C. affinis is an Amazonian species, thus its occurrence in the state of São Paulo is improbable. Therefore, C. affinis is removed from the fauna of São Paulo.

\section{Cephaloleia dimidiaticornis Baly, 1869 (Imatidiini)}

Published record. São Paulo (Staines \& García-Robledo 2014).

Distribution. Brazil (? Amazonas) and Peru.

Remarks. Cephaloleia dimidiaticornis was described from Peru and afterwards cited only in catalogues. STAINES \& GARCÍA-Robledo (2014) published specimens from the Peruvian Amazon and 'São Paulo' without any further data. If the locality data and identification are correct, I assume that it must refer to São Paulo de Olivença which was frequently confused with the state of São Paulo by various authors. Alternatively, the specimen was either mislabelled or misidentified but I am quite sure that the species does not occur in the state of São Paulo as it is morphologically similar to some other Amazonian species. Therefore, the record is here tentatively placed as from São Paulo de Olivença in the state of Amazonas and the species is removed from the fauna of São Paulo.

\section{Cephaloleia obsoleta Weise, 1910 (Imatidiini)}

Published record. 'Cipo’ (Staines \& García-Robledo 2014).

Distribution. Brazil.

Remarks. Until recently, Cephaloleia obsoleta was known only based on the holotype specimen with locality data 'Brasilia'. StaInes \& GARcía-Robledo (2014) published two specimens from São Paulo: Cipo deposited in E. Riley's collection. I did not examine them in hand, but Ed Riley provided me with a photograph of these specimens. They certainly do not belong to $C$. obsoleta but to some other small black coloured species as they have acompletely different shape of body. Therefore, the species is excluded from the fauna of São Paulo.

\section{Cephaloleia ornata Waterhouse, 1881 (Imatidiini)}

Published record. Boraceia (STAINES \& GARCÍA-Robledo 2014).

Distribution. Bolivia, ? Brazil (Pará), Colombia, Ecuador, Peru, and Venezuela.

Remarks. StAINES \& GARCÍA-ROBLEDO (2014) recorded this species from Brazil for the first time but did not formally mention that. They published material from Taperina, which was unassigned to the state, and then from Boraceia in the state of São Paulo. I did not have the opportunity to examine this material but based on the presently known distribution, the species is found in the Upper Amazon basin including foothills of the Andes and thus its occurrence in São Paulo is very improbable. In general, any species of the group, where $C$. ornata belongs does not occur in eastern Brazil.
Therefore, the material must have been either mislabelled or misidentified, and C. ornata is removed from the fauna of São Paulo.

On the other hand, occurrence of $C$. ornata in the Brazilian Amazon is probable. As for the other specimen(s), Taperina is most likely an alternative spelling of Taperinha, which is close to Santarém in Pará. However, STAINeS \& GARCÍA-ROBLEDO (2014) did not provide any additional data for this specimen(s), thus it would be necessary to verify the labels and identification to confirm the occurrence of $C$. ornata in Brazil. For now, I leave the distribution in Brazil with a question mark.

\section{Cephaloleia ornatula Donckier, 1899 (Imatidiini)}

Distribution. Brazil (Rio de Janeiro).

Remarks. Until recently, this species was known only based on two syntypes with locality data 'America Meridional'. StAINES \& GARCíA-Robledo (2014) published two specimens from the state of Rio de Janeiro; however, in the line with the species distribution São Paulo only is mentioned. This is obviously a mistake and the species should not be listed as present in São Paulo because there is no formal record. The other possibility would be that they recorded C. ornata from São Paulo and placed the record under the wrong name.

\section{Chlamydocassis (Ceratocassis) bicornuta (Boheman, 1850) (Goniocheniini)}

Published record. Indiana (Buzzi 1988, = C. laticollis, see remarks under that species).

Distribution. Brazil (Bahia, Ceará, Minas Gerais, Pernambuco) and ? Colombia.

Remarks. Distribution of C. bicornuta is here revised as some authors considered $C$. laticollis a synonym of $C$. bicornuta (e.g., SpAETH 1931, Buzzi 1988); thus, the distribution of the two taxa was mixed. I did not have the opportunity to examine the material Buzzi studied but very likely some of these specimens belong to C. laticollis and were published earlier by Viana (1964b). Chlamydocassis bicornuta was described from Bahia and Colombia. Further records from Bahia, Ceará and Minas Gerais (VIANA 1964) are reliable as Viana studied the type material of both taxa. I found two paralectotypes from Colombia in the Zoological Museum in Copenhagen (coll. Westermann) and both morphologically agree with the lectotype; however; their locality data are questionable as such a vicariant distribution is very improbable. Remaining records belong to $C$. laticollis, and thus São Paulo, Paraguay and Venezuela are removed from the range of $C$. bicornuta.

\section{Coptocycla (s. str.) undecimpunctata (Fabricius, 1781) (Cassidini)}

Published record. 'S. Paulo' (Spaeth 1914).

Distribution. Brazil (Amapá, Pará) and French Guiana. Remarks. In the Coleopterorum Catalogus, SpAeth (1914) stated the distribution of $C$. undecimpunctata as 'Cayenne, S. Paulo'. At his time, the species was well known from French Guiana but there was no published record from 
São Paulo and no other record exists. It is impossible to find out whether this was a simple mistake or Spaeth had some material from São Paulo and published the record in Coleopterorum Catalogus, which is, however, very unusual as Spaeth did not publish faunistic records unless it was a report from some expedition. On the other hand, the specimen reported here as C. stigma was identified by Spaeth as ' $C$. undecimpunctata var.', thus he might have some other specimens. Also, it is possible that the locality does not refer to the state of São Paulo but to the municipality of São Paulo de Olivença, where C. undecimpunctata could theoretically occur. The species is known only from the eastern part of the Guiana shield and its occurrence in the state of São Paulo is very improbable.

\section{Heterispa vinula (Erichson, 1847) (Chalepini)}

Published record. São Paulo-Ipiranga (CASARI \& TEIXEIRA 2004).

Distribution. Bolivia, Brazil (Amazonas), Costa Rica, Colombia, Ecuador, Mexico, Nicaragua, Panama, Peru, and Venezuela.

Remarks. The species was recorded from São Paulo based on misidentification with $H$. costipennis, see Remarks under the latter species.

\section{Microctenochira difficilis (Boheman, 1855) (Cassidini)}

Distribution. Brazil (Bahia, Paraná, Rio de Janeiro), Ecuador, and Peru.

Remarks. Presence of $M$. difficilis in the state of São Paulo was published by TEIXEIRA \& CASARI (2003); however, this record proved to be misidentification of Eremionycha bahiana, see ŚWIĘTOJAŃSKa (2009: 79). Therefore, M. difficilis is accordingly removed from the fauna of São Paulo. On the other hand, its occurrence in São Paulo is probable.

\section{Oxychalepus posticatus (Baly, 1885) (Chalepini)}

Published record. Santos (Weise 1921, Staines 2010).

Distribution. Costa Rica, Nicaragua, and Panama.

Remarks. The species was recorded only once from Santos in São Paulo based on historic material (WeISE 1921). STAINES (2010) repeated part of the same material. Otherwise, the species is known only from the southern part of Central America. Such disjunct distribution is very improbable and thus $O$. posticatus is removed from the fauna of Brazil.

\section{Paraselenis (Pseudechoma) marginipennis (Spaeth, 1907) (Mesomphaliini)}

Published record. 'Mendes [= collector; thus, São Paulo only]' (BoROWIEC 1996).

Distribution. Bolivia, Ecuador, and Peru.

Remarks. BorowiEC (1996) published material labelled 'Sao Paulo Mendes' and interpreted Mendes as the name of locality. However, it is most likely a name of collector rather than locality (L. Borowiec, pers. comm. 2020) and thus, the record is here interpreted as São Paulo only.

Moreover, occurrence of this species in São Paulo is dubious as P. marginipennis is distributed in the foothills (up to ca. $1000 \mathrm{~m}$ ) of the Andes, primarily in Peru and N Bolivia. Therefore, the species is removed from the fauna of Brazil as the only record is the one from São Paulo.

\section{Sceloenopla bidens (Fabricius, 1792) (Sceloenoplini)}

Distribution. Brazil (Amazonas), Colombia, Ecuador, French Guiana, Peru, and Suriname.

Remarks. Costa et al. (1988) published description of larva of Sceloenopla af. bidens (Fabricius, 1792) based on material collected in Campinas but did not discuss further the identification. LOURENÇÃo et al. (1991) published biological observations on the same population of $S$. bidens and provided colour photograph of the adult. Regarding identification, they wrote following: 'Specimens were sent in 1984 to Systematic Entomology Laboratory, USDA and identified by Dr. R. E. White as S. bidens or very close species. In 1989, Dr. Cleide Costa compared these specimens to the holotype of $S$. bidens in MNHN and concluded that they are the same species.' I have repeatedly tried to locate the type of S. bidens in MNHN and failed; however, it was described from Suriname and the species is common in the Guiana shield. The specimen figured by LoURENÇÃo et al. (1991) shows high and broad costae on the elytra matching another species, S. rectelineata. I had at disposal numerous specimens of $S$. bidens from French Guiana and historical material from MNHN and BMNH identified as this species; they have distinctly lower and narrower costae in comparison to the holotype of $S$. rectelineata. Therefore $S$. bidens is removed from the fauna of São Paulo and the respective records are transferred to $S$. rectelineata. True $S$. bidens is with certainty distributed in the Guiana shield, other records should be verified.

\section{Sternostena varians Weise, 1910 (Chalepini)}

Published record. São Paulo (MonRós \& VIANA 1947).

Distribution. Argentina, Brazil (Minas Gerais, Rio de Janeiro, Santa Catarina, São Paulo), Peru, Suriname, and Trinidad and Tobago.

Remarks. MONRÓS \& ViAnA (1947) mentioned occurrence in São Paulo for S. varians ab. apicalis Weise, 1910; however, this record probably refers to the material published by UHMANN (1932) as $S$. varians ab. triangularis and thus the species Sternostena varians Weise, 1910 was never recorded from São Paulo, although its occurrence there is possible.

\section{Stolas hermanni (Spaeth, 1911) (Mesomphaliini)}

Published record. Itapecirica da Serra (BOROWIEC \& TAKIZAWA 2011).

Distribution. Ecuador and Peru.

Remarks. BOROWIEC \& TAKIZAWA (2011) recorded two specimens of S. hermanni from São Paulo, Brazil; however, I find it quite unlikely that they truly were collected in $\mathrm{E}$ Brazil and in my opinion, they were erroneously labelled. Stolas hermanni belongs among the species characterized by usually more or less visible pubescence on elytra, disc of elytra usually black and explanate margin with more or less developed yellow/red/orange pattern. There are several species-groups with these characters, and all are restricted 
to the Andes and sub-Andean zones, particularly in Ecuador and Peru. So far, the easternmost of these species is $S$. agenysiformis Borowiec, 2011 from Rondônia. Therefore, $S$. hermanni is removed from the fauna of São Paulo. The same case is $S$. pleurosticha and S. scoparia recorded in the same publication; all three species are common in Peru. Moreover, all three were recorded from the same locality and collecting date and are the only published from this locality by BOROWIEC \& TAKIZAWA (2011); thus, I am quite sure they must have been mislabelled.

\section{Stolas pleurosticha (Erichson, 1847) (Mesomphaliini)}

Published record. Itapecerica da Serra (BorowIEC \& TAKIZAWA 2011).

Distribution. Bolivia, Colombia, Ecuador, and Peru.

Remarks. This is the same case as $S$. hermanni and therefore the species is removed from the fauna of Brazil.

\section{Stolas scoparia (Erichson, 1847) (Mesomphaliini)}

Published record. Itapecerica da Serra (BorowIEC \& TAKIZAWA 2011).

Distribution. Bolivia, Brazil (Mato Grosso), and Peru.

Remarks. Hincks (1956) recorded this species from Brazil for the first time based on three specimens collected in Rio de Janeiro-Corcovado by F. Tippmann. Borowiec (1996) recorded two more specimens from Chapada in Mato Grosso. Finally, BorowIEC \& TAKIZAWA (2011) recorded two specimens from São Paulo. The records from Rio de Janeiro and São Paulo are certainly erroneous due to mislabelled specimens and are removed from the species distribution; see also Remarks in S. hermanni. On the other hand, the record from Chapada is possible. The respective specimens were collected by H. H. Smith and the locality refers to Chapada dos Guimarães (CALVERT 1909), which is about $500-600 \mathrm{~km}$ air distance from the localities of $S$. scoparia in Bolivia.

\section{Summary of changes in species distribution}

\section{Tribe Alurnini}

Alurnus batesii Baly, 1864: Brazil (São Paulo) - excluded, probably erroneous interpretation of locality data $=$ Amazonas

Alurnus grossus Fabricius, 1775: Brazil (São Paulo) - excluded, erroneous interpretation of locality data or mislabelled material

\section{Tribe Cassidini}

Agroiconota subvittata (Boheman, 1855): Brazil (São Paulo) - excluded, misidentification as A. urbanae

Agroiconota tristriata (Fabricius, 1792): Brazil (São Paulo) - new record Aporocassida graphica (Germar, 1823): Brazil (São Paulo) - dubious record, requires confirmation by recent material

Charidotella (Philaspis) polita (Klug, 1829): Brazil (Rio Grande do Sul) new interpretation of the type locality; Brazil (São Paulo) - new record

Charidotis admirabilis Boheman, 1855: Brazil (São Paulo) - new record Charidotis auroguttata Boheman, 1855: Brazil (São Paulo) - new record Charidotis brevicollis Spaeth, 1936: Brazil (São Paulo) - dubious, probably erroneous locality data

Charidotis circumscripta Boheman, 1855: Brazil (São Paulo) - new record Charidotis concentrica (Boheman, 1855): Brazil (São Paulo) - new record Charidotis consentanea (Boheman, 1855): Brazil (São Paulo) - new record Charidotis gemellata Boheman, 1855: Brazil (São Paulo) - new record Coptocycla (s. str.) stigma (Germar, 1823): Brazil (São Paulo)-new record
Coptocycla (s. str.) undecimpunctata (Fabricius, 1781): Brazil (São Paulo) - excluded, erroneous locality data or misidentification

Coptocycla (Coptocyclella) adamantina (Germar, 1823): Brazil (São Paulo) - new record

Eremionycha bahiana (Boheman, 1855): Brazil (São Paulo) - new record

Helocassis flavorugosa (Boheman, 1855): Brazil (São Paulo) - new record

Metriona elatior (Klug, 1829): French Guiana - excluded, erroneous locality data

Metrionella calva (Boheman, 1855): Brazil (São Paulo) - new record

Microctenochira difficilis (Boheman, 1855): Brazil (São Paulo) - excluded, misidentification as Eremionycha bahiana

Microctenochira patruelis (Boheman, 1855): Brazil (São Paulo) - new record

Plagiometriona deyrollei (Boheman, 1855): Brazil (São Paulo) - new record

Plagiometriona punctatissima (Boheman, 1855): Brazil (São Paulo) - new record, lapsus under P. punctipennis [sic!]

Plagiometriona tenella (Klug, 1829): Brazil (São Paulo) - new record

\section{Tribe Chalepini}

Baliosus conspersus Weise, 1911: Brazil (São Paulo) - new record Chalepus aenescens Weise, 1910: Brazil (São Paulo) - new record Decatelia pallipes (Weise, 1922): Brazil (São Paulo) - new record

Heterispa vinula (Erichson, 1847): Brazil (São Paulo) - excluded, misidentification as $H$. costipennis

Octhispa gemmata (Germar, 1823): Brazil (São Paulo) - new record Octhispa robinsonii (Baly, 1864): Brazil (São Paulo) - new record Octotoma brasiliensis Weise, 1921: Brazil (São Paulo) - new record Octotoma crassicornis Weise, 1910: Brazil (São Paulo) - new record

Oxychalepus anchora (Chapuis, 1877): Brazil (São Paulo) - dubious records, probably misidentifications

Oxychalepus centralis Uhmann, 1940: Brazil (São Paulo) - new record

Oxychalepus posticatus (Baly, 1885): Brazil (São Paulo) - excluded, misidentification or erroneous locality data

Physocoryna scabra Guérin-Méneville, 1844: Brazil (Pará) - excluded, misinterpretation of locality data $=$ São Paulo

Sternostena varians Weise, 1910: Brazil (São Paulo) - excluded, erroneous interpretation $=S$. triangularis

Temnochalepus imitans Uhmann, 1935: Brazil (São Paulo) - new record Uroplata coarctata Weise, 1921: Brazil (São Paulo) - new record

Uroplata minuscula (Chapuis, 1877): Brazil (São Paulo) - new record

\section{Tribe Goniocheniini}

Chlamydocassis (Ceratocassis) bicornuta (Boheman, 1850): Brazil (São Paulo), Paraguay, and Venezuela - misidentification as C. laticollis; Colombia - most likely erroneous locality data

Chlamydocassis (Ceratocassis) laticollis (Boheman, 1850): Brazil (Bahia) - excluded, misinterpretation of C. bicornuta

Chlamydocassis (s. str.) perforata (Boheman, 1850): Brazil (Rio Grande do Sul, São Paulo) - doubtful, requires verification of the respective specimens and confirmation by a new material

Goniochenia (s. str.) parvula Weise, 1896: French Guiana - excluded, erroneous locality data

\section{Tribe Imatidiini}

Calliaspis cinnabarina Boheman 1850: Brazil (Rio de Janeiro, São Paulo) - doubtful records, require confirmation by new material

Calliapis umbonata Hincks, 1956: Brazil (São Paulo) - new record

Cephaloleia affinis Baly, 1859: Brazil (São Paulo) - excluded, probably erroneous interpretation of locality data = Amazonas

Cephaloleia caeruleata Baly, 1875: Brazil (Santa Catarina) - excluded, misidentification as C. impressa; Brazil (São Paulo) - new record; Ecuador - excluded, misidentification or erroneous locality data

Cephaloleia deyrollei Baly, 1859: Bolivia, Ecuador and French Guianaexcluded, misidentifications or erroneous locality data

Cephaloleia dimidiaticornis Baly, 1869: Brazil (São Paulo) - excluded, probably erroneous interpretation of locality data $=$ Amazonas or misidentification 
Cephaloleia elaeidis Maulik, 1924: Brazil (São Paulo), Ecuador-dubious records, require verification of material

Cephaloleia flavovittata Baly, 1859: Brazil (São Paulo) - new record

Cephaloleia fulvipes Baly, 1859: Ecuador - excluded, probably misidentified or erroneous locality data

Cephaloleia marantae Uhmann, 1957: Brazil (Amazonas) - excluded, misinterpretation of locality data

Cephaloleia obsoleta Weise, 1910: Brazil (São Paulo) - excluded, misidentification

Cephaloleia ornata Waterhouse, 1881: Brazil (São Paulo) - excluded, probably misidentification or erroneous locality data

Cephaloleia ornatula Donckier, 1899: Brazil (São Paulo) - excluded, erroneous interpretation

Cephaloleia trilineata Uhmann, 1942: Brazil (Minas Gerais, São Paulo) - new records

Cephaloleia vittipennis Weise, 1910: Argentina - dubious, requires confirmation by new material; Peru and Venezuela - excluded, misidentifications or erroneous locality data

Cephaloleia zikani Uhmann, 1935: Brazil (São Paulo) - new record Stenispa vespertina Baly, 1877: Brazil (São Paulo) - new record Stenispa viridis (Pic, 1931): Brazil (São Paulo) - new record

Xenispa bicolorata (Uhmann, 1948): Brazil (São Paulo) - new record

\section{Tribe Mesomphaliini}

Agenysa caedemadens (Lichtenstein, 1796): Brazil (São Paulo)-dubious, requires confirmation by new material

Anacassis candida (Boheman, 1854): Brazil (São Paulo) - new record

Anacassis punctulata (Klug, 1829): Brazil (Rio Grande do Sul) - new interpretation of the type locality

Chelymorpha commutabilis Boheman, 1854: Brazil (São Paulo) - new record

Chelymorpha constellata (Klug, 1829): Brazil (Rio Grande do Sul) - new interpretation of the type locality; Brazil (São Paulo) - new record

Cyrtonota cyanea (Linnaeus, 1758): Peru - excluded, erroneous locality data

Cyrtonota sexpustulata (Fabricius, 1781): Peru - excluded, erroneous locality data

Cyrtonota similata (Boheman, 1850): Brazil (Minas Gerais, Rio de Janeiro) - excluded, misidentifications as C. vulnerata

Cyrtonota vulnerata (Boheman, 1850): Brazil (Minas Gerais, São Paulo) - new records

Hilarocassis evanida (Boheman, 1850): Brazil (São Paulo) - new record

Mesomphalia gibbosa (Fabricius, 1781): Brazil (São Paulo) - new record

Nebraspis corticina (Boheman, 1850): Brazil (São Paulo) - new record

Omaspides (s. str.) iheringi (Spaeth, 1909): Peru-dubious record, possibly misidentification

Paraselenis (Pseudechoma) marginipennis (Spaeth, 1907): Brazil (São Paulo) - excluded, erroneous locality data

Stolas acuta (Boheman, 1850): Brazil (São Paulo) - new record

Stolas aenea (Olivier, 1790): Brazil (São Paulo) - new record; French Guiana - excluded, erroneous locality data

Stolas hermanni (Spaeth, 1911): Brazil (São Paulo) - excluded, erroneous locality data

Stolas pleurosticha (Erichson, 1847): Brazil (São Paulo) - excluded, erroneous locality data

Stolas scoparia (Erichson, 1847): Brazil (Rio de Janeiro, São Paulo) excluded, erroneous locality data

Stolas sexsignata (Boheman, 1850): Brazil (São Paulo) - new record

Stolas sommeri (Boheman, 1850): Brazil (São Paulo) - new record

Stolas subreticulata (Boheman, 1850): Brazil (São Paulo) - new record

\section{Tribe Omocerini}

Cyclosoma (Dolichotoma) fuscopunctatum (Spaeth, 1919): Peru - excluded, probably erroneous locality data

Cyclosoma (Dolichotoma) germari (Spaeth, 1913): Brazil (Amazonas), French Guiana - excluded, probably erroneous locality data

Cyclosoma (Dolichotoma) mitior (Boheman, 1850): Brazil (Amazonas) - excluded, erroneous interpretation of locality data = Pará; Brazil (Pará) - new record but dubious locality data
Omocerus (Platytauroma) cornutus (Boheman, 1850): Brazil (São Paulo) - new record

Omocerus (Platytauroma) truncatus (Boheman, 1850): Brazil (Amazonas), Bolivia, Colombia, French Guiana - excluded, erroneous locality data or misidentifications

Tribe Spilophorini

Calyptocephala nigricornis (Germar, 1823): Brazil (São Paulo) - new record

Oediopalpa brunnea (Uhmann, 1943): Brazil (São Paulo) - new record Oediopalpa caerulescens (Baly, 1875): Brazil (São Paulo) - new record Oediopalpa fulvipes Baly, 1859: Brazil (São Paulo) - new record

\section{Acknowledgements}

I would like to express my sincere thanks to Lech Borowiec (Wrocław, Poland) for his consultations and permanent support; Ed Riley (Texas, USA), who kindly provided information and photographs of two problematic species of Cephaloleia; Gabriel Biffi and Gustavo Rizzi (MZUSP), Michele Leocádio (Universidade Federal do Rio de Janeiro), and Marianna Simões (SDEI) are thanked for providing scans of many Brazilian publications, essential for completion of this catalogue; Jan Bezděk (Brno), Lech Borowiec, and Petr Kment (Prague) are thanked for critical reading and valuable feedback; Jitka Ansari (Bedford) is thanked for correction of English. My special thanks go to my family, Katka and Matyáš, for patience with my beetle studies and for support. The study was partly financially supported by the Ministry of Culture of the Czech Republic (DKRVO 2019-2023/5.I.b, National Museum, 00023272).

\section{References}

ALBERTONI F. F. 2017: Morphology and natural history of two species of bromeliad leaf beetles in the genus Calliaspis Dejean, 1836 from Southern Brazil, with a summary of the current knowledge of Imatidiini immatures (Coleoptera: Chrysomelidae: Cassidinae). Zootaxa 4312: 113-142.

BALY J. S. 1859: Catalogue of Hispidae in the collection of the British Museum. Part I. Printed by order of the trustees of the British Museum, London, 172 pp. +9 pls.

BALY J. S. 1875: Descriptions of hitherto uncharacterized species of Phytophaga. Entomologist's Monthly Magazine 12: 73-75.

BOHEMAN C. H. 1850: Monographia Cassididarum. Tomus Primus. Officina Norstedtiana, Holmiae, 452 pp. +4 pls

BOHEMAN C. H. 1854: Monographia Cassididarum. Tomus Secundus. Officina Norstedtiana, Holmiae, 506 pp. +2 pls.

BOHEMAN C. H. 1855: Monographia Cassididarum. Tomus Tertius. Officina Norstedtiana, Holmiae, 543 pp. +1 pl.

BOHEMAN C. H. 1857: Catalogue of Coleopterous Insects in the collection of the British Museum. Part IX. Cassida. Printed by Order of the trustees [of the British Museum], London, $225 \mathrm{pp}$.

BOHEMAN C. H. 1862: Monographia Cassididarum. Tomus Quartus. (Supplementum). Officina Norstedtiana, Holmiae, vi +504 pp.

BONDAR G. 1913: A praga do 'Alurnus' ou 'Barata do Coqueiro'. Chacaras e Quintaes 8: 52.

BONDAR G. 1922: Insectos damninhos e molestias do coqueiro (Cocos nucifera) no Brasil. Imprensa Official do Estado, Bahia, 111 pp.

BONDAR G. 1938: Notas entomologicas da Bahia II. Revista de Entomologia 8: 15-24.

BONDAR G. 1940: Insectos nocivos e molestias do coqueiro (Cocos nucifera) no Brasil. Boletim do Instituto Central de Fomento Economico de Bahia 8: 1-160.

BOROWIEC L. 1989: Three new species of Charidotella Weise (Coleoptera, Chrysomelidae, Cassidinae), with checklist of the genus. Polskie Pismo Entomologiczne 59: 203-222. 
BOROWIEC L. 1996: Faunistic records of Neotropical Cassidinae (Coleoptera: Chrysomelidae). Polskie Pismo Entomologiczne 65: 119-251.

BOROWIEC L. 1999: A world catalogue of the Cassidinae (Coleoptera: Chrysomelidae). Biologica Silesiae, Wrocław, 476 pp.

BOROWIEC L. 2002: New records of Neotropical Cassidinae, with description of three new species (Coleoptera: Chrysomelidae). Genus 13: $43-138$.

BOROWIEC L. 2005: Six new species of the genus Agroiconota Spaeth, 1913 (Coleoptera: Chrysomelidae: Cassidinae), with a key to the genus. Annales Zoologici (Warszawa) 55: 61-74.

BOROWIEC L. 2009: New records of Neotropical tortoise beetles (Coleoptera: Chrysomelidae: Cassidinae). Genus 20: 615-722.

BOROWIEC L. \& MORAGUES G. 2005: Tortoise beetles of the French Guyana - a faunistic review (Coleoptera: Chrysomelidae: Cassidinae) Genus 16: 247-278.

BOROWIEC L. \& POMORSKA J. 2009: The structure of the spermatecae of the genus Stolas (Coleoptera: Chrysomelidae: Cassidinae: Mesomphaliini) and its taxonomic significance. Annales Zoologici (Warszawa) 59: 201-221.

BOROWIEC L. \& SKUZA M. 2004: The structure of spermatheca in the genus Chelymorpha Chevrolat, 1837 (Coleoptera: Chrysomelidae: Cassidinae) and its taxonomic significance. Annales Zoologici (Warszawa) 54: 439-451.

BOROWIEC L. \& ŚWIĘTOJAŃSKA J. 2020: Cassidnae of the world - an interactive manual (Coleoptera: Chrysomelidae). Permanent electronic publication available from: http://www.cassidae.uni. wroc.pl $/ \mathrm{katalog} \% 20$ internetowy/index.htm (last accessed on $30^{\text {th }}$ July 2020).

BOROWIEC L. \& TAKIZAWA H. 2011: Neotropical tortoise beetles in the Amazon Insectarium, Tokyo, Japan with description of nine new species (Coleoptera: Chrysomelidae: Cassidinae). Genus 22: 427-484.

BOSQ J. M. 1943: Segunda lista de Coleópteros de la república Argentina daninos a la agricultura. Ingeniería Agronómica 4: 7-80.

BOTTCHER A., ZOLIN J. P., NOGUEIRA-DE-SÁ F. \& TRIGO J. R. 2009: Faecal shield chemical defense is not important in larvae of the tortoise beetle Chelymorpha reimoseri (Chrysomelidae: Cassidinae: Stolaini). Chemoecology 19: 63-66.

BUCK P. 1958: Hispinae (Coleopt. Chrysomelidae) in der entomologischen Sammlung des Instituto Anchietano de Pesquisas, Porto Alegre. Pesquisas 2: 145-152.

BUZZI J. Z. 1988: Biology of Neotropical Cassidinae. Pp. 559-580. In: JOLIVET P., PETITPIERRE E. \& HSIAO T. H. (eds): Biology of Chrysomelidae. Kluwer Academic Publishers, Dordrecht-Boston -London, xxiv +615 pp.

BUZZI J. Z. 1994: Host plants of Neotropical Cassidinae. Pp. 205-212. In: JOLIVET P., COX M. \& PETITPIERRE E. (eds): Novel aspects of the biology of Chrysomelidae. Kluwer Academic Publishers, Dordrecht-Boston-London, xxiii + $582 \mathrm{pp}$.

BUZZI J. Z. 1996: A new species of Agroiconota Spaeth from Brazil (Coleoptera: Chrysomelidae: Cassidinae). Coleopterists Bulletin 50: 1-4

BUZZI J. Z. 1999: Uma nova espécie de Euctenochira Hincks (Coleoptera, Chrysomelidae, Cassidinae), da Serra do Japi, Jundiaí, Sao Paulo, Brasil. Revista Brasileira de Entomologia 16: 771-773.

BUZZI Z. J. \& MIYAZAKI R. D. 1999: Description of immatures and life cycle of Stolas lacordairei (Boheman) (Coleoptera, Chrysomelidae, Cassidinae). Pp. 581-597. In: COX M. L. (ed.): Advances in Chrysomelidae Biology 1. Backhuys Publishers, Leiden, xii +671 pp.

CALVERT P. P. 1909: Contributions to a knowledge of the Odonata of the Neotropical region, exclusive of Mexico and Central America. Annals of the Carnegie Museum 6: 73-280 + 9 pls.

CASARI S. A. 2005: Larva and pupa of Metaxycera purpurata (Chrysomelidae, Cassidinae, Chalepini). Iheringia, Série Zoologia 95: 373-376.

CASARI S. A. \& QUEIROZ J. M. 2005: Description of two leaf-miner larvae (Coleoptera: Chrysomelidae: Cassidinae) from the Brazilian Atlantic forest. Zootaxa 1081: 47-59.

CASARI S. A. \& TEIXEIRA E. P. 2004: Immatures of Heterispa vinula (Erichson) and Physocoryna scabra Guérin-Méneville (Coleoptera, Chrysomelidae, Cassidinae, Chalepini). Revista Brasileira de Entomologia 48: 473-480.
CASARI S. A. \& TEIXEIRA E. P. 2010: Immatures of Gratiana conformis (Boheman) (Coleoptera, Chrysomelidae, Cassidinae). Revista Brasileira de Entomologia 54: 235-242.

CHABOO C. S. 2001: Revision and phylogenetic analysis of Acromis Chevrolat (Coleoptera: Chrysomelidae: Cassidinae: Stolaini). Coleopterists Bulletin 55: 75-102.

CHABOO C. S. 2002: Range extensions of the new world tortoroise beetles (Coleoptera: Chrysomelidae: Cassidinae). Proceedings of the Entomological Society of Washington 104: 716-720.

COSTA C., VANIN S. A. \& CASARI-CHEN S. A. 1988: Larvas de coleoptera do Brasil. Museu de Zoologia, São Paulo, v + 282 pp. +165 pls.

COUTO SOARES A. J. Do C. 1962: Revisão monográfica do sub-gênero Platytauroma, com a descrição de uma nova espécie. Arquivos do Museu Nacional 52: 219-225.

DESCARPENTRIES A. \& VILLIERS A. 1959: Les types de la collection M. Pic. I. Chrysomelinae et Hispinae du nouveau-monde. Bulletin du Museum National d'Histoire Naturelle Paris 31: 137-154.

FERNANDES F. R. \& BOROWIEC L. 2013: Review of the genus Cassidinoma Hincks (Coleoptera: Chrysomelidae: Cassidinae: Omocerini). Zootaxa 3620: 544-552.

FISCHER C. R. 1935: Os coleopteros phytophagos da tribu Alurnini, pragas das palmeiras (Chrysomelidae, Hispinae). Revista de Entomologia (Rio de Janeiro) 5: 257-292.

FLINTE V., BOROWIEC L., FERITAS S., VIANA J. H., FERNANDES F. R., NOGUEIRA-DE-SÁ F., MACEDO M. V. \& MONTEIRO R. F. 2009: Tortoise beetles of the State of Rio de Janeiro, Brazil (Coleoptera: Chrysomelidae: Cassidinae). Genus 20: 571-614.

HAITLINGER R. 1991: Canestriniid mites (Acari, Astigmata, Canestriniidae) occurring on Chrysomelidae (Insecta, Coleoptera) in Neotropical Region. I. Genera Grandiellina Fain, Arrunsithiana n. gen. Wiadomości Parazytologiczne 37: 381-399.

HORN W. \& KAHLE I. 1935: Über entomologische Sammlungen, Entomologen \& Entomo-Museologie (Ein Beitrag zur Geschichte der Entomologie). Teil I. Entomologische Beihefte (Dahlem) 2: i-vi $+1-160+16$ pls.

HINCKS W. D. 1952: The genera of the Cassidinae (Coleoptera: Chrysomelidae). Transactions of the Royal Entomological Society of London 103: 327-358.

HINCKS W. D. 1956: Some Neotropical Cassidinae (Col., Chrysomelidae) in the Museum G. Frey. Entomologischen Arbeiten aus dem Museum G. Frey 7: 545-559.

ICZN 1999: International code of zoological nomenclature. Fourth edition. The International Trust for Zoological Nomenclature, London, xxix +306 pp.

JUNG C. S. \& MONNÉ M. L. 2006: Coraliomela brunnea brunnea: morfologia do adulto e sinonímias (Coleoptera, Chrysomelidae). Iheringia, Série Zoologia 96: 377-381.

KLUG J. C. F. 1829: Preis-Verzeichniss vorräthiger Insectendoubleten des Königl. zoologischen Museums der Universität. Zoologisches Museum, Berlin, 18 pp.

LOPES T. A., FERNANDES F. R. \& SCHNEIDER M. C. 2016: Comparative cytogenetic analysis in 13 tortoise beetles (Coleoptera: Chrysomelidae: Cassidinae) from Brazil. European Journal of Entomology 113: 352-363.

LOURENÇÃO A. L., STEIN C. P. \& MATTHES L. A. F. 1991: Aspectos biológicos de Sceloenopla bidens, praga de filodendros. Bragantia 50: $77-82$.

LÜDERWALDT H. 1910: Die Frasspuren von Cephaloldia deyrollei Baly. Zeitschrift für Wissenschaftliche Insektenbiologie 6: 61-63.

MENDES L. O. T. 1938: Relação dos insétos encontrados sobre plantas do Estado de S. Paulo nos anos de 1936-1937. Revista de Agricultura 13: 482-490.

MONRÓS F. \& VIANA M. J. 1947: Revisión sistemática de los Hispidae argentinos (Insecta, Coleopt. Chrysomeloid.). Annales del Museo Argentino de Ciencias Naturales Bernardino Rivadavia 42: 125-324 +27 pls.

NOGUEIRA-DE-SÁ F. \& TRIGO J. R. 2002: Do fecal shields provide physical protection to larvae of the tortoise beetles Plagiometriona flavescens and Stolas chalybea against natural enemies? Entomologia Experimentalis et Applicata 104: 203-206. 
NOGUEIRA-DE-SÁ \& TRIGO J. R. 2005: Faecal shield of the tortoise beetle Plagiometriona aff. flavescens (Chrysomelidae: Cassidinae) as chemically mediated defence against predators. Journal of Tropical Ecology 21: 189-194.

NOGUEIRA-DE-SÁ F. \& VASCONCELLOS-NETO J. 2003a: Natural enemies of Neotropical cassidinae (Coleoptera: Chrysomelidae) and their phenology. Pp. 161-174. In: JOLIVET P. \& FURTH D. G. (eds): Special topics in leaf beetle biology. Proceedings of the Fifth International Symposium on the Chrysomelidae. Pensoft Publishers, Sofia, xii +339 pp.

NOGUEIRA-DE-SÁ F. \& VASCONCELLOS-NETO J. 2003b: Host plant utilization and population abundance of three tropical species of Cassidinae (Coleoptera: Chrysomelidae). Journal of Natural History 37: 681-696.

OBENBERGER J. 1922: Sběry p. J. Mráze v Národním museu. [Collection of Mr. J. Mráz in the National Museum]. Časopis Musea Království Českého, Oddíl Př́rodovědný 96: 93-94 (in Czech).

OBENBERGER J. 1927a: Jaro Mráz. Časopis Národního Musea, Oddíl Prírodovědný 101: 65-68 (in Czech).

OBENBERGER J. 1927b: Jaro Mráz. Časopis Československé Společnosti Entomologické 24: 77-80 (in Czech).

PIC M. 1929: Nouveautés diverses. Mélanges Exotico-Entomologiques 54: $1-36$

PIC M. 1932: Nouveautés diverses. Mélanges Exotico-Entomologiques 59: 10-36.

PIC M. 1948: Coléoptères du globe (suite). L'Échange, Revue Linnéenne 64: 9-12.

RAMOS T. C. 1996: Revisão do genero Clinocarispa Uhmann, 1935 (Coleoptera, Chrysomelidae, Hispinae, Chalepini). Revista Brasileira de Entomologia 40: 367-374.

RAMOS T. C. 1998: Revisão das espécies brasileiras de Oxychalepus Uhmann, 1937 (Coleoptera, Chrysomelidae, Hispinae). Revista Brasileira de Entomologia 41: 305-315.

SEKERKA L. 2010: Icones Insectorum Europae Centralis. Coleoptera: Chrysomelidae: Cassidinae. Folia Heyrovskiana, Series B 13: 1-24.

SEKERKA L. 2014: Review of Imatidiini genera (Coleoptera: Chrysomelidae: Cassidinae). Acta Entomologica Musei Nationalis Pragae 54: $257-314$.

SEKERKA L. 2016: Taxonomic and nomenclatural changes in Cassidinae (Coleoptera: Chrysomelidae). Acta Entomologica Musei Nationalis Pragae 56: 275-344.

SEKERKA L. 2020: Cassidinae in Catálogo Taxonômico da Fauna do Brasil. Permanent electronic publication available from: http:// fauna.jbrj.gov.br/fauna/faunadobrasil/115879 (last accessed on $30^{\text {th }}$ July 2020).

SEKERKA L. \& BARCLAY M. L. V. 2014: Fabrician types of Cassidinae (Coleoptera: Chrysomelidae) deposited in the Natural History Museum, London. Acta Entomologica Musei Nationalis Pragae 54: 657-684.

SIMÕES M. V. P. 2014: Taxonomic revision of the genus Paranota Monrós and Viana, 1949 (Coleoptera: Chrysomelidae: Cassidinae: Dorynotini). Coleopterists Bulletin 68: 631-655.

SIMÕES M. V. P. \& MONNÉ M. L. 2008: New records of South American Cassidinae (Coleoptera: Chrysomelidae). Genus 19: 709-715.

SIMÕES M. V. P. \& MONNÉ M. L. 2011: Inventário das espéces de Cassidinae (Insecta, Coleoptera, Chrysomelidae) do Parque Nacional do Itatiaia, RJ, Brasil. Biota Neotropica 11: 215-228.

SIMÕES M. V. P. \& MONNÉ M. L. 2014: Taxonomic revision of the genus Mesomphalia Hope, 1839 (Insecta, Coleoptera, Chrysomelidae). Zootaxa 3835: 151-197.

SIMÕES M. V. P. \& SEKERKA L. 2015: Review of the Neotropical leaf beetle subgenus Dorynota s. str. Chevrolat (Coleoptera: Chrysomelidae: Cassidinae: Dorynotini). Coleopterists Bulletin 69: 231-254.

SOUZA LOPES H. de 1941: Relatório de terceira excursao a zona de Estrada de Ferro Noroeste do Brazil realizada em Fevreiro e Marco de 1940. IV - Relacao do material entomológico capturado. Memoiras do Instituto Oswaldo Cruz 35 [1940]: 641-659.

SPAETH F. 1905: Beschreibung einiger neuer Cassiden nebst synonymischen Bemerkungen V. Verhandlungen der Kaiserlich-Königlichen Zoologisch-Botanischen Gesellschaft in Wien 55: 79-118.

SPAETH F. 1909: Beschreibung einiger neuer Cassiden nebst synonymi- schen Bemerkungen VII. Verhandlungen der Kaiserlich-Königlichen Zoologisch-Botanischen Gesellschaft in Wien 59: 364-397.

SPAETH F. 1913: Kritische Studien über den Umfang und die Begrenzung mehrerer Cassiden-Gattungen nebst Beschreibung neuer amerik. Arten. Archiv für Naturgeschichte 79: 126-164.

SPAETH F. 1914: Chrysomelidae: 16. Cassidinae. In: SCHENKLING S. (ed.) Coleopterorum Catalogus. Pars 62. W. Junk, Berlin, 180 pp.

SPAETH F. 1919: Drei Neue Cassidinen aus dem tropischen Amerika. Neue Beiträge zur Systematischen Insektenkunde 1: 121-123.

SPAETH F. 1923: Neue Cassidinen (Coleoptera) des tropischen Amerika. Norsk Entomologisk Tidsskrift 1 [1922]: 165-181.

SPAETH F. 1926a: Beschreibung neuer Cassidinen. Bulletins Mensuels de la Société des Naturalistes Luxembourgeois, Nouvelle Série 20 : 11-24, 47-60.

SPAETH F. 1926b: Monographie der zu Gruppe der Coptocyclitae gehörigen amerikanischen Cassidinen (Col.): I. Die Gattungen mit gekämmten Klauen. Supplementa Entomologica 13: 1-108.

SPAETH F. 1926c: Mitteilungen ueber die Cassidinen des Prager Nationalmuseums (Col. Chrysomelidae). Sborník Entomologického Oddělení Národního Musea v Praze 4: 81-96.

SPAETH F. 1928: Mitteilungen ueber die Cassidinen des Prager Nationalmuseums II (Col. Chrysomelidae). Sborník Entomologického Odděleni Národního Musea v Praze 6: 29-47.

SPAETH F. 1931: Wissenschaftliche Ergebnise der Schwedischen Entomologischen Reisen des Herrn Dr. A. Roman in Amazonas und Bahia 1914-15 und 1923-24. Cassidinae. Entomologisk Tidskrift 53: $183-187$.

SPAETH F. 1932a: Neue Cassiden (Col. Chrysom.). Stettiner Entomologische Zeitung 93: 182-204.

SPAETH F. 1932b: Über die mit Metriona erratica verwandten Arten (Col., Chrysom. Cass.). Folia Zoologica et Hydrobiologica 4: 263-270.

SPAETH F. 1935: Mitteilungen über neue oder bemerkenswerte Cassidinen aus dem Senckenberg-Museum (Ins. Col.). Entomologische Rundschau 53: 65-69.

SPAETH F. 1936a: Bestimmungstabelle der Gattung Charidotis (Col. Chrys. Cassid.). Sborník Entomologického Oddělení Národního Musea v Praze 14: 66-99.

SPAETH F. 1936b: Die Untergattungen von Coptocycla mit gerieften Klauen. Pp. 251-262. In: Festschrift zum 60. Geburtstage von Professor Dr. Embrik Strand. Vol. 1. Izdevnieciba Latvija, Riga, ii +644 pp. +14 pls.

SPAETH F. 1937: Neue Cassidinen des Rijksmuseums in Leiden und meiner Sammlung. Temminckia 2: 135-158.

SPAETH F. 1940: Ueber die Gattung Poecilaspis Hope (Coleoptera, Cassidinae). Temminckia 5: 143-162.

STAINES C. L. 1988: A review of the species of Acritispa Uhmann (Coleoptera: Chrysomelidae, Hispinae). Proceedings of the Entomological Society of Washington 90: 193-195.

STAINES C. L. 1994: A revision of the genus Anisostena Weise (Coleoptera: Chrysomelidae, Hispinae) Part III. The pilatei species group. Insecta Mundi 8: 213-226.

STAINES C. L. 1998: A review of the genus Physocoryna Guérin-Méneville, 1844 (Coleoptera: Chrysomelidae: Hispinae). Journal of the New York Entomological Society 106: 163-169.

STAINES C. L. 2002: The New World tribes and genera of Hispines (Coleoptera: Chrysomelidae: Cassidinae). Proceedings of the Entomological Society of Washinghton 104: 721-784.

STAINES C. L. 2007: A new species of Platyauchenia Stürm, 1843 (Coleoptera: Chrysomelidae: Cassidinae) from Brazil. Insecta Mundi 12: $1-7$.

STAINES C. L. 2010: A review of the genus Oxychalepus Uhmann, 1937 (Coleoptera: Chrysomelidae: Cassidinae). Zootaxa 2573: 35-56.

STAINES C. L. 2013: A review of the genus Alurnus Fabricius, 1775 (Coleoptera: Chrysomelidae: Cassidinae). Insecta Mundi 291: 1-34.

STAINES C. L. 2014: A revision of the genus Acentroptera Guérin-Méneville, 1844 (Coleoptera: Chrysomelidae: Cassidinae). Insecta Mundi 332: 1-19.

STAINES C. L. \& GARCÍA-ROBLEDO C. 2014: The genus Cephaloleia Chevrolat, 1836 (Coleoptera, Chrysomelidae, Cassidinae). ZooKeys 436: $1-355$. 
ŚWIĘTOJAŃSKA J. 2002: Revision of the genera Aporocassida Spaeth, 1952 and Saulaspis Spaeth, 1913 (Coleoptera: Chrysomelidae: Cassidinae). Annales Zoologici (Warszawa) 52: 573-581.

ŚWIĘTOJAŃSKA J. 2009: The immatures of tortoise beetles with review of all described taxa (Coleoptera: Chrysomelidae: Cassidinae). Polish Taxonomical Monographs 16: 1-158.

ŚWIĘTOJAŃSKA J. \& BOROWIEC L. 1999: Two new species of Microctenochira Spaeth from Brazil and Peru (Coleoptera: Chrysomelidae: Cassidinae). Genus 6: 447-454.

TEIXEIRA E. P. \& CASARI S. A. 2003: Descriptions and biological notes of immatures of Microctenochira difficilis (Coleoptera, Chrysomelidae, Hispinae, Cassidini). Iheringia, Séria Zoologia 93: 23-30.

TOLEDO PIZA S. DE, JR. 1968: Insetos do Piracicaba. Universidade de São Paulo, Piracicaba, 123 pp +44 pls.

UHMANN E. 1927: Hispinen des Deutschen Entomologischen Institutes Berlin-Dahlem. 3. Beitrag zur Kenntnis der Hispinen. Entomologische Blätter 23: 58-65.

UHMANN E. 1930: Amerikanische Hispinen (Col. Chrys.). 19. Beitrag zur Kenntnis der Hispinen. Entomologische Blätter 26: 33-28.

UHMANN E. 1931: Südamerikanische Hispinen aus dem deutschen Entomologischen Institut Berlin-Dahlem. 30. Beitrag zur Kenntnis der Hispinen (Col. Chrys.). Bolletino della Società Entomologica Italiana 63: 58-61.

UHMANN E. 1932: Hispinen aus Brasilien gesammelt von Herrn Pfarrer Kessel. 36. Beitrag zur Kenntnis der Hispinen (Col. Chrys.). Mitteilungen der Deutschen Entomologischen Gesellschaft, E.V. 3: 36-38.

UHMANN E. 1936: Amerikanische Hispinen aus dem Zoologischen Museum der Universität Berlin. V. Teil. Die Gattung Chalepus i.sp. Thunberg. 61. Beitrag zur Kenntnis der Hispinen (Col. Chrys.). Pp. 613-629. In: Festschrift zum 60. Geburtstage von Professor Dr. Embrik Strand. Vol. 1. Izdevnieciba Latvija, Riga, ii +644 pp. +14 pls.

UHMANN E. 1937a: Übersicht über die ersten Gattungen der Uroplatini (Col. Chrys.) (67. Beitrag zur Kenntnis der Hispinen). Entomologische Blätter 33: 336-337.

UHMANN E. 1937b: Amerikanische Hispinen aus dem Zoologischen Museum der Universitat Berlin. VI. Teil. Die Gattung Uroplata Chap. 68. Beitrag zur Kenntnis der Hispinen (Col. Chrys.). Pp. 451-467. In: Festschrift zum 60. Geburtstage von Professor Dr. Embrik Strand. Vol. III. Izdevnieciba Latvija, Riga, ii +608 pp. +20 pls.

UHMANN E. 1938a: Amerikanische Hispinen aus dem Zoologischen Museum der Universität Berlin. VII. Teil: Die Gattung Xenochalepus Weise. 70. Beitrag zur Kenntnis der Hispinen (Col. Chrys.). Revista de Entomologia 8: 420-440.

UHMANN E. 1938b: Hispinen aus dem Britischen Museum. I. Teil. 71. Beitrag zur Kenntnis der Hispinen (Coleoptera Chrysomelidae). Proceedings of the Royal Entomological Society of London, Series B 7: 109-116

UHMANN E. 1938c: Deckenzeichnung von Stethispa crenatula n. sp. Südamerikanische Hispinen aus dem Zoologischen Museum der Universität Berlin. IV. Teil. Chalepini vor Chalepus. 58. Beitrag zur Kenntnis der Hispinen (Col. Chrys.). Revista Argentina de Entomologia 1: 69-72.

UHMANN E. 1939: Amerikanische Hispinen aus dem Berliner Museum. IX. Teil. Die Gattungen Octhispa Chap, Baliosus Ws., Probaenia Ws., Acanthodes Baly, Cnestispa Mlk. und Pentispa Chap. 77. Beitrag zur Kenntnis der Hispinen (Col. Chrys). Pp. 317-345. In: Festschrift zum 60. Geburtstage von Professor Dr. Embrik Strand. Vol. V. Izdevnieciba Latvija, Riga, ii +750 pp. +11 pls.

UHMANN E. 1940: Die Genotypen der von mir aufgestellten HispinenGattungen. 88. Beitrag zur Kenntnis der Hispinen (Col. Chrys.) Entomologisk Tidskrift 61: 143-144.
UHMANN E. 1948a: Neue Hispinae aus Suedamerika (Col. Chrysom.). 107. Beitrag zur Kenntnis der Hispinae. Revista de Entomologia 19: 207-230.

UHMANN E. 1948b: Über Hispinae (Ins. Col.) des SenckenbergMuseums. 112. Beitrag zur Kenntnis der Hispinae (Chrysomel.). Senckenbergiana 29: 73-76.

UHMANN E. 1957: Chrysomelidae: Hispinae. Hispinae Americanae. In: HINCKS W. D. (ed.): Coleopterorum Catalogus Supplementa, Pars 35, Fasc. 1. Dr. W. Junk, 's-Gravenhage, vii + pp. 1-153.

UHMANNE. 1960: Über Hispinae(Ins. Col.) des Senckenberg-Museums. II. Teil. 204. Beitrag zur Kenntnis der Hispinae (Chrysomelidae). Senckenbergiana Biologica 41: 303-305.

UHMANN E. 1961: Hispinae aus Verschiedenen Erdteilen. 202. Beitrag zur Kenntnis der Hispinae (Coleoptera, Chrysomelidae). Pesquisas, Serie Zoologia 10: 1-63.

UHMANN E. 1964: Hispinae aus dem Staate Sao Paulo, Brasilien (209. Beitrag zur Kenntnis der Hispinae (Coleoptera, Chrysomelidae). Iheringia, Séria Zoologia 32: 1-27.

UHMANN E. 1965: Insecta Amapaensia: Hispinae (Col., Chrysomelidae). 218. Beitrag zur Kenntnis der Hispinae. Papéis Avulsos do Departamento de Zoologia 17: 113-117.

UHMANN E. 1969: Neue Hispinae aus Brasilien (232. Beitrag zur Kenntnis der Hispinae; Coleoptera, Chrysomelidae). Papéis Avulsos de Zoologia 21: 125-130.

VASCONCELLOS-NETO J. 1988: Genetics of Chelymorpha cribraria, Cassidinae: colour patterns and their ecological meanings. Pp. 217-232. In: JOLIVET P., PETITPIERRE E. \& HSIAO T. H. (eds): Biology of Chrysomelidae. Kluwer Academic Publishers, Dordrecht-Boston-London, xxiv $+615 \mathrm{pp}$.

VIANA J. M. 1964a: Revision sistematica de las especies Argentinas de Omocerini Spaeth y catalogo bibliografico de la tribus (Coleopt., Chrysomelidae, Cassidinae). Revista del Museo Argentino de Ciencas Naturales Bernardino Rivadavia 1: 1-131 + 10 pls.

VIANA J. M. 1964b: Revision sistematica de las especies Argentinas de Goniocheniini Spaeth y catalogo bibliografico de la tribus (Coleopt., Chrysomelidae, Cassidinae). Revista del Museo Argentino de Ciencas Naturales Bernardino Rivadavia 1: 217-266 + 5 pls.

VIANA J. M. 1968: Revision sistematica de las Eugenysini Spaeth con nuevas especies y catalogo bibliografico de la tribus (Coleopt., Chrysomelidae, Cassidinae). Revista del Museo Argentino de Ciencas Naturales Bernardino Rivadavia 3: $1-106+6$ pls.

WEISE J. 1896: Beschreibung neuer Cassida-Arten und synonymische Bemerkungen. Deutsche Entomologische Zeitschrift 1896: 15-32.

WEISE J. 1900: Eine neue Alurnus-Form. Deutsche Entomologische Zeitschrift 1900: 218-219.

WEISE J. 1905: Beschreibung einiger Hispinen. Archiv für Naturgeschichte 71: 49-104.

WEISE J. 1906: Hispinae, Coccinelidae et Endomychidae Argentina et veciniate e Collectione Bruchiana. Revista del Museo de la Plata 12: 221-231.

WEISE J. 1910a: Beitrag zur Kenntnis der amerikanischen Hispinen. Archiv für Naturgeschichte 76: 67-127.

WEISE J. 1910b: Zweiter Beitrag zur Kenntnis der amerikanischen Hispinen. Verhandlungen des Naturforschenden Vereines in Brünn 48: $115-162$.

WEISE J. 1911: Dritter Beitrag zur Kenntnis der Hispinen. Annales de la Société Entomologique de Belgique 55: 39-78.

WEISE J. 1921: Amerikanische Hispinen. Archiv für Naturgeschichte, Abteilung A 87: 263-275. 
